\title{
Asymmetric Reductive Coupling of Dienes and Aldehydes Catalyzed by Nickel Complexes of Spiro Phosphoramidites: Highly Enantioselective Synthesis of Chiral Bishomoallylic Alcohols
}

Yun Yang, Shou-Fei Zhu, Hai-Feng Duan, Chang-Yue Zhou, Li-Xin Wang, and Qi-Lin Zhou* State Key Laboratory and Institute of Elemento-organic Chemistry, Nankai University Tianjin 300071, China

\section{CONTENTS}

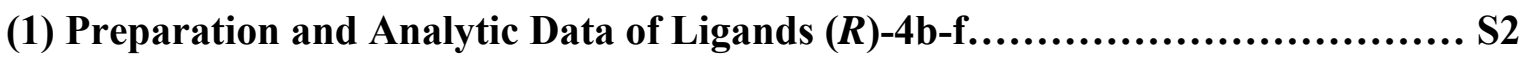

(2) General Procedure for Asymmetric Reductive Coupling Reaction................ S9

(3) Analytical Data of Chiral Bishomoallylic Alcohols 3...........................S10

(4) NMR Spectra for New Ligands............................................. S17

(5) NMR Spectra and HPLC Charts for Chiral Bishomoallylic Alcohols 3........... S29

\section{General:}

The reactions and manipulations which are sensitive to moisture or air were performed in an argon-filled glove box (VAC DRI-LAB HE 493) or using standard Schlenk techniques. Commercially available reagents were used as received without further purification unless otherwise noticed. $\mathrm{Ni}(\mathrm{COD})_{2}$ was purchased from Strem Chemicals, Inc. Ni(acac) $)_{2}$ was purchased from Acros Chemicals, Inc. and used without further purification. Diethylzinc (2.4 M solution in toluene) was prepared by adding neat diethylzinc to the fresh-distilled toluene. Anhydrous tetrahydrofuran and toluene were distilled from sodium benzophenone ketyl under nitrogen. Ligands SIPHOS and ShiP can be obtained from Strem Chemicals, Inc. Other ligands were prepared from $(R)-1,1^{\prime}$-Spirobiindane-7,7'-diol $((R)$-SPINOL). NMR spectra were recorded with a Bruker or Varian spectrometer at $300 \mathrm{MHz}\left({ }^{1} \mathrm{H} \mathrm{NMR}\right), 75 \mathrm{MHz}\left({ }^{13} \mathrm{C}\right.$ $\mathrm{NMR})$ and $121.5 \mathrm{MHz}\left({ }^{31} \mathrm{P} \mathrm{NMR}\right)$. Chemical shifts were reported in ppm down field from 
internal $\mathrm{Me}_{4} \mathrm{Si}$ and external $85 \% \mathrm{H}_{3} \mathrm{PO}_{4}$, respectively. Optical rotations were measured using a Perkin Elmer Model 341 polarimeter. Elemental analyses were performed on Yanaca CDRDER MT-3 instrument. Mass spectra were recorded on a VG-7070E spectrometer. GC analyses were performed using a Hewlett Packard Model HP 6890 Series. HPLC analyses were performed using a Hewlett Packard Model HP 1100 Series or Waters 2996 chromatography.

\section{(1) Preparation and Analytical Data of Ligands (R)-4b-f}
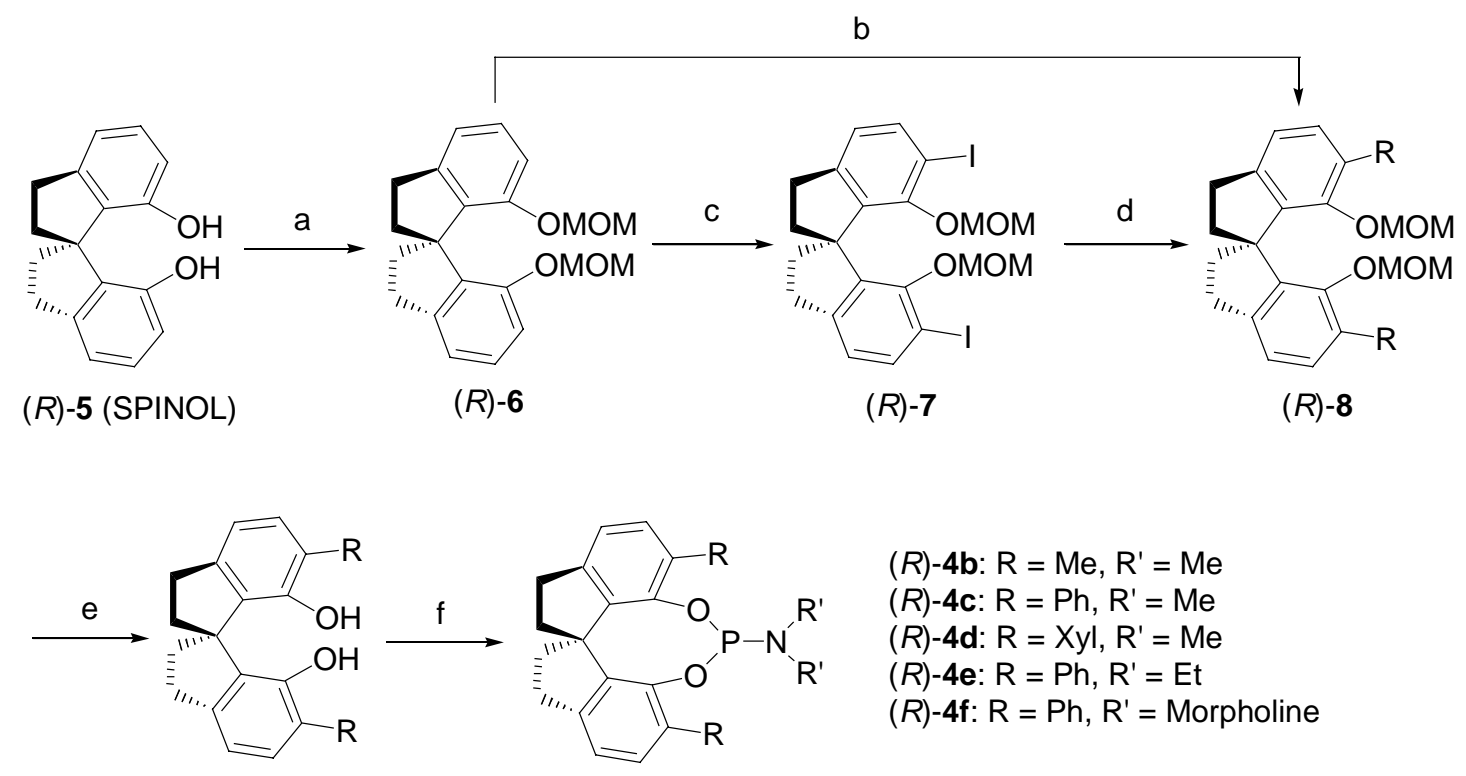

(R)-9

Scheme 1. Reagents and conditions: (a) $\mathrm{NaH}, \mathrm{ClCH}_{2} \mathrm{OCH}_{3}$, THF, $0{ }^{\circ} \mathrm{C}$ to rt, $95 \%$; (b) $n$-BuLi, TMEDA,

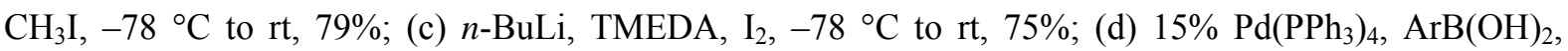
aqueous $\mathrm{K}_{2} \mathrm{CO}_{3}$, THF/MeOH, reflux, 77-94\%; (e) Conc. $\mathrm{HCl}, \mathrm{CHCl}_{3} / \mathrm{MeOH}$, reflux, 93-97\%; (f) 4b-d: HMPT, toluene, reflux, 70-77\%; 4e: HEPT, toluene, reflux, $63 \%$; 4f: (i) $\mathrm{PCl}_{3}, \mathrm{Et}_{3} \mathrm{~N}, \mathrm{THF},-78^{\circ} \mathrm{C}$ to rt; (ii) lithated morpholine, $\mathrm{Et}_{3} \mathrm{~N},-30{ }^{\circ} \mathrm{C}$ to rt, $62 \%$.

\section{Synthesis of $(R)-7,7^{\prime}$-bis(1-methoxymethoxy)-1,1'-spirobiindane $((R)-6)$}

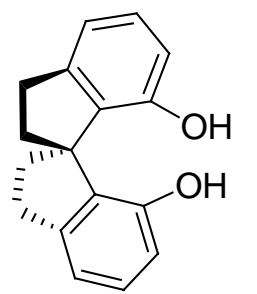

$(R)$-SPINOL

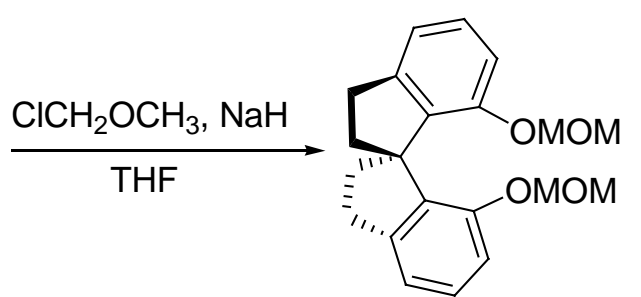

$(R)-6$

To an oven-dried three-necked round-bottom flask charged with $\mathrm{NaH}(0.96 \mathrm{~g}, 40 \mathrm{mmol})$ and $15 \mathrm{~mL}$ THF, a solution of $(R)-1,1^{\prime}$-spirobiindane-7,7'-diol $((R)$-SPINOL) $(2.0 \mathrm{~g}, 8 \mathrm{mmol})$ in $10 \mathrm{~mL}$ THF was added slowly at $0{ }^{\circ} \mathrm{C}$ with stirring. The resulted mixture was stirred for $3 \mathrm{~h}$ 
and chloro(methoxy)methane $(1.5 \mathrm{~mL}, 19.2 \mathrm{mmol})$ in $10 \mathrm{~mL}$ THF was added slowly at $0{ }^{\circ} \mathrm{C}$. After stirred at ambient temperature for $6 \mathrm{~h}$, the mixture was diluted with ethyl acetate and quenched with a small amount of water. The organic phase was separated, washed with saturated $\mathrm{NH}_{4} \mathrm{Cl}$ and brine, and dried over $\mathrm{MgSO}_{4}$. After removal of solvent, the residue was purified by chromatography (ethyl acetate/petroleum ether, $\mathrm{v} / \mathrm{v}=1: 8)$ to give $(R)-6(2.58 \mathrm{~g}$, 95\% yield) as colorless oil. $[\alpha]^{20}{ }_{\mathrm{D}}+23.1\left(\mathrm{c} 1.0, \mathrm{CHCl}_{3}\right) ;{ }^{1} \mathrm{H} \mathrm{NMR}\left(300 \mathrm{MHz}, \mathrm{CDCl}_{3}\right) \delta 7.06$ (t, $J=7.8 \mathrm{~Hz}, 2 \mathrm{H}, \mathrm{Ar}-\mathrm{H}), 6.87$ (d, $J=7.2 \mathrm{~Hz}, 2 \mathrm{H}, \mathrm{Ar}-\mathrm{H}), 6.72(\mathrm{~d}, J=8.1 \mathrm{~Hz}, 2 \mathrm{H}, \mathrm{Ar}-\mathrm{H}), 4.83$ $\left(\mathrm{dd}, J=21.6\right.$ and $\left.6.3 \mathrm{~Hz}, 4 \mathrm{H}, \mathrm{CH}_{2}\right), 3.14-2.95\left(\mathrm{~m}, 10 \mathrm{H}, \mathrm{CH}_{3}\right.$ and $\left.\mathrm{CH}_{2}\right), 2.51-2.41(\mathrm{~m}, 2 \mathrm{H}$, $\left.\mathrm{CH}_{2}\right), 2.23-2.15\left(\mathrm{~m}, 2 \mathrm{H}, \mathrm{CH}_{2}\right) ;{ }^{13} \mathrm{C}$ NMR $\left(75 \mathrm{MHz}, \mathrm{CDCl}_{3}\right) \delta 153.6,145.8,137.7,127.7$, 117.8, 114.4, 93.6, 59.6, 55.5, 39.3, 31.9; MS (ESI) m/z 340; Anal. Calcd for $\mathrm{C}_{21} \mathrm{H}_{24} \mathrm{O}_{4}$ : C 74.09, H 7.11; Found: C 74.17, H 7.21.

\section{Synthesis of $(R)-6,6^{\prime}$-diiodo-7,7'-bis(1-methoxymethoxy)-1,1'-spirobiindane $((R)-7)$}

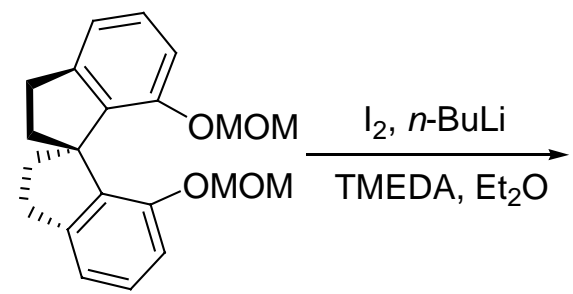

$(R)-6$

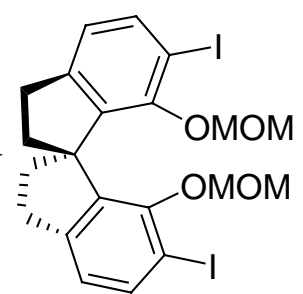

$(R)-7$

To a solution of $(R)-6(2.07 \mathrm{~g}, 6 \mathrm{mmol})$ and tetramethylethylenediamine $(1.9 \mathrm{ml}, 12.6 \mathrm{mmol})$ in $20 \mathrm{~mL}$ ether, $8.2 \mathrm{~mL}(18 \mathrm{mmol})$ of $2.2 \mathrm{M} \mathrm{n}$-BuLi in hexane was added under $\mathrm{N}_{2}$ at $-78^{\circ} \mathrm{C}$. The mixture was stirred at ambient temperature for $4.0 \mathrm{~h}$, and then was cooled to $-78{ }^{\circ} \mathrm{C}$ again. Iodine ( $4.6 \mathrm{~g}$ in $25 \mathrm{ml}$ ether) was added slowly. The mixture was warmed to ambient temperature and stirred overnight, $100 \mathrm{ml}$ of a saturate solution of $\mathrm{Na}_{2} \mathrm{SO}_{3}$ in water was cautiously added. The mixture was stirred for additional $1 \mathrm{~h}$, the organic layer was separated and aqueous layer was extracted by ether. The organic phase was combined and dried over $\mathrm{MgSO}_{4}$. After removal of solvent, the residue was purified by chromatography (ethyl acetate/petroleum ether: $\mathrm{v} / \mathrm{v}=1 / 15)$ to give $(R)-7(2.66 \mathrm{~g}, 75 \%$ yield $)$ as a white solid. Mp $125-128^{\circ} \mathrm{C},[\alpha]^{20}{ }_{\mathrm{D}}+175\left(\mathrm{c} 1.0, \mathrm{CHCl}_{3}\right) ;{ }^{1} \mathrm{H}$ NMR $\left(300 \mathrm{MHz}, \mathrm{CDCl}_{3}\right) \delta 7.62(\mathrm{~d}, J=8.1 \mathrm{~Hz}$, $2 \mathrm{H}, \mathrm{Ar}-\mathrm{H}), 6.77(\mathrm{~d}, J=7.8 \mathrm{~Hz}, 2 \mathrm{H}, \mathrm{Ar}-\mathrm{H}), 4.85\left(\mathrm{~d}, J=4.8 \mathrm{~Hz}, 2 \mathrm{H}, \mathrm{CH}_{2}\right), 4.63(\mathrm{~d}, J=5.1 \mathrm{~Hz}$, $\left.2 \mathrm{H}, \mathrm{CH}_{2}\right), 3.03-2.99\left(\mathrm{M}, 4 \mathrm{H}, \mathrm{CH}_{2}\right), 2.96\left(\mathrm{~s}, 6 \mathrm{H}, \mathrm{CH}_{3}\right), 2.52-2.41\left(\mathrm{~m}, 2 \mathrm{H}, \mathrm{CH}_{2}\right), 2.22-2.14(\mathrm{~m}$, $\left.2 \mathrm{H}, \mathrm{CH}_{2}\right) ;{ }^{13} \mathrm{C} \mathrm{NMR}\left(75 \mathrm{MHz}, \mathrm{CDCl}_{3}\right) \delta 154.2,146.8,143.6,138.8,122.6,99.4,89.0,61.2$, 57.0, 39.5, 31.0; HRMS (EI) Calcd for $\mathrm{C}_{21} \mathrm{H}_{22} \mathrm{I}_{2} \mathrm{O}_{4}$ : 591.9607; Found 591.9592.

\section{Synthesis of $(R)-6,6^{\prime}$-dimethyl-1,1'-spirobiindane-7,7'-bis(1-methoxymethoxy) $((R)-8 b)$}




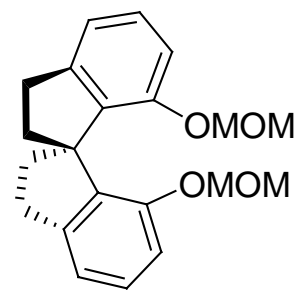

$(R)-6$

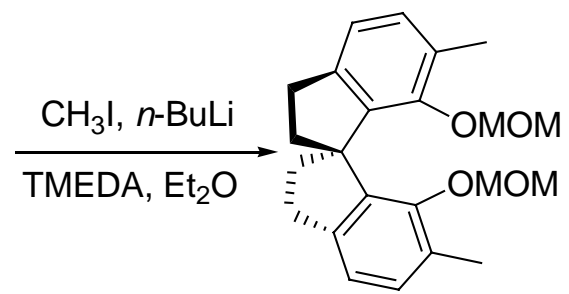

$(R)-8 b$

To a solution of $(R)-6(517 \mathrm{mg}, 1.50 \mathrm{mmol})$ and tetramethylethylenediamine $(0.88 \mathrm{~mL}, 5.76$ $\mathrm{mmol})$ in $30 \mathrm{~mL}$ ether, $2.62 \mathrm{~mL}(5.76 \mathrm{mmol})$ of $2.2 \mathrm{M} \mathrm{n}$-BuLi in hexane was added under $\mathrm{N}_{2}$ at $-78{ }^{\circ} \mathrm{C}$. After stirred at ambient temperature for $4 \mathrm{~h}$, the suspension was cooled to $-78{ }^{\circ} \mathrm{C}$, and the MeI $(878 \mathrm{mg}, 6.0 \mathrm{mmol})$ in $15 \mathrm{~mL}$ ether was added slowly. After the addition was completed, the mixture was warmed to ambient temperature and stirred for another $5.0 \mathrm{~h}$ before a saturated aq. $\mathrm{NH}_{4} \mathrm{Cl}(10 \mathrm{~mL})$ was added cautiously. The organic layer was then separated and aqueous layer was extracted by ether. The organic phase was combined and dried over $\mathrm{MgSO}_{4}$. After removal of solvent, the residue was purified by chromatography (ethyl acetate/petroleum ether: $\mathrm{v} / \mathrm{v}=1: 15)$ to give $(R)-8 \mathbf{b}(434 \mathrm{mg}, 79 \%$ yield) as colorless oil. $[\alpha]^{20}{ }_{\mathrm{D}}+168\left(\mathrm{c} 1.0, \mathrm{CH}_{2} \mathrm{Cl}_{2}\right)$; ${ }^{1} \mathrm{H}$ NMR $\left(300 \mathrm{MHz}, \mathrm{CDCl}_{3}\right) \delta 7.01(\mathrm{~d}, J=7.5 \mathrm{~Hz}, 2 \mathrm{H}, \mathrm{Ar}-\mathrm{H})$, $6.92(\mathrm{~d}, J=7.5 \mathrm{~Hz}, 2 \mathrm{H}, \mathrm{Ar}-\mathrm{H}), 4.47\left(\mathrm{~d}, J=5.4 \mathrm{~Hz}, 2 \mathrm{H}, \mathrm{CH}_{2}\right), 4.23\left(\mathrm{~d}, J=5.4 \mathrm{~Hz}, 2 \mathrm{H}, \mathrm{CH}_{2}\right)$, 3.09-2.93 (m, 10H, $\mathrm{CH}_{3}$ and $\left.\mathrm{CH}_{2}\right), 2.57-2.47\left(\mathrm{~m}, 2 \mathrm{H}, \mathrm{CH}_{2}\right), 2.41-2.15\left(\mathrm{~m}, 8 \mathrm{H}, \mathrm{CH}_{3}\right.$ and $\left.\mathrm{CH}_{2}\right) ;{ }^{13} \mathrm{C} \mathrm{NMR}\left(75 \mathrm{MHz}, \mathrm{CDCl}_{3}\right) \delta 152.8,143.5,142.6,130.3,129.0,120.2,98.4,59.9,56.4$, 40.5, 31.1, 16.9; HRMS (EI) Calcd for $\mathrm{C}_{24} \mathrm{H}_{28} \mathrm{O}_{4}$ : 368.1987; Found 368.1985.

\section{Synthesis of $(R)-6,6^{\prime}$-diphenyl-7,7'-bis(1-methoxymethoxy)-1,1'-spirobiindane $((R)-8 c)$}

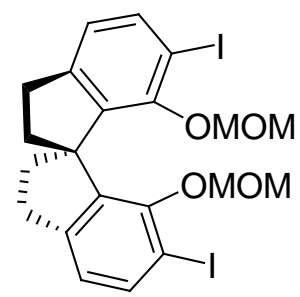

$(R)-7$
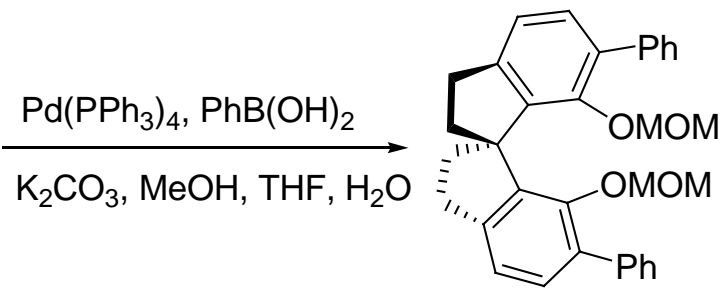

$(R)-8 \mathrm{C}$

To a solution of $(R)-7(2.4 \mathrm{~g}, 4.1 \mathrm{mmol})$, phenylboronic acid $(2.0 \mathrm{~g}, 16.4 \mathrm{mmol})$ and $\mathrm{Pd}\left(\mathrm{PPh}_{3}\right)_{4}(710 \mathrm{mg}, 0.62 \mathrm{mmol})$ in THF $(120 \mathrm{~mL})$ and $\mathrm{MeOH}(5 \mathrm{~mL})$, aqueous $\mathrm{K}_{2} \mathrm{CO}_{3}(2 \mathrm{M}$, $13 \mathrm{~mL}$ ) was added. The reaction mixture was heated at reflux for $22 \mathrm{~h}$ and then the mixture was cooled to ambient temperature, diluted by $\mathrm{CH}_{2} \mathrm{Cl}_{2}$, washed with brine, dried over $\mathrm{MgSO}_{4}$ and purified by column chromatography (ethyl acetate/petroleum ether, $\mathrm{v} / \mathrm{v}=1 / 15$ ) to give (R)-8c $(1.9 \mathrm{~g}, 94 \%$ yield $)$ as a white solid. Mp 116-117 ${ }^{\circ} \mathrm{C},[\alpha]^{20}{ }_{\mathrm{D}}+256\left(\mathrm{c} 1.03, \mathrm{CH}_{2} \mathrm{Cl}_{2}\right) ;{ }^{1} \mathrm{H}$ NMR $\left(300 \mathrm{MHz}, \mathrm{CDCl}_{3}\right) \delta 7.52-7.50(\mathrm{~m}, 4 \mathrm{H}, \mathrm{Ar}-\mathrm{H}), 7.40-7.35(\mathrm{~m}, 4 \mathrm{H}, \mathrm{Ar}-\mathrm{H}), 7.30-7.24(\mathrm{~m}$, 2H, Ar-H), 7.14 (d, J=7.5 Hz, 2H, Ar-H), 7.04 (d, J=7.5 Hz, 2H, Ar-H), 4.35 (d, J=5.1 Hz, $\left.2 \mathrm{H}, \mathrm{CH}_{2}\right), 4.21\left(\mathrm{~d}, J=5.1 \mathrm{~Hz}, 2 \mathrm{H}, \mathrm{CH}_{2}\right), 3.11-3.06\left(\mathrm{~m}, 4 \mathrm{H}, \mathrm{CH}_{2}\right), 2.71\left(\mathrm{~s}, 6 \mathrm{H}, \mathrm{CH}_{3}\right)$, 2.61-2.51 (m, 2H, $\left.\mathrm{CH}_{2}\right), 2.36-2.30\left(\mathrm{~m}, 2 \mathrm{H}, \mathrm{CH}_{2}\right) ;{ }^{13} \mathrm{C} \mathrm{NMR}\left(75 \mathrm{MHz}, \mathrm{CDCl}_{3}\right) \delta 151.9,145.4$, 
$142.6,140.0,133.1,130.5,129.3,128.4,126.9,120.3,98.6,60.2,56.4,39.5,31.3$; MS (FAB) m/z 492.2; Anal. Calcd for $\mathrm{C}_{33} \mathrm{H}_{32} \mathrm{O}_{4}$ : C 80.46, H 6.55; Found: C 80.16, H 6.52.

\section{Synthesis of $(R)-6,6^{\prime}$-di(3,5-dimethylphenyl)-7,7'-bis(1-methoxymethoxy)-1,1'- spirobiindane $((R)-8 d)$}

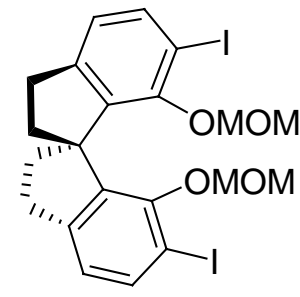

$(R)-7$

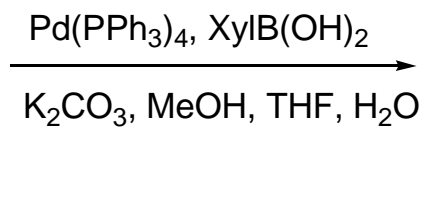

$(R)-8 d$

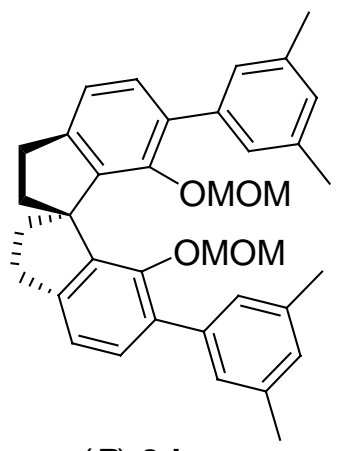

The compound $(R)-\mathbf{8 d}$ was prepared $77 \%$ yield by the same procedure as that for $(R)-\mathbf{8 c}$ using 3,5-dimethylphenylboronic acid as a foamy solid. $[\alpha]^{20}{ }_{\mathrm{D}}+107\left(c \quad 0.51, \mathrm{CH}_{2} \mathrm{Cl}_{2}\right) ;{ }^{1} \mathrm{H} \mathrm{NMR}$ $\left(400 \mathrm{MHz}, \mathrm{CDCl}_{3}\right) \delta 7.12-7.10(\mathrm{~m}, 6 \mathrm{H}, \mathrm{Ar}-\mathrm{H}), 7.01(\mathrm{~d}, J=7.6 \mathrm{~Hz}, 2 \mathrm{H}, \mathrm{Ar}-\mathrm{H}), 6.91(\mathrm{~s}, 2 \mathrm{H}$, $\mathrm{Ar}-\mathrm{H}), 4.33\left(\mathrm{~d}, J=5.2 \mathrm{~Hz}, 2 \mathrm{H}, \mathrm{CH}_{2}\right), 4.16\left(\mathrm{~d}, \mathrm{~J}=5.2 \mathrm{~Hz}, 2 \mathrm{H}, \mathrm{CH}_{2}\right), 3.09-3.06\left(\mathrm{~m}, 4 \mathrm{H}, \mathrm{CH}_{2}\right)$, $2.73\left(\mathrm{~s}, 6 \mathrm{H}, \mathrm{CH}_{3}\right), 2.59-2.52\left(\mathrm{~m}, 2 \mathrm{H}, \mathrm{CH}_{2}\right), 2.32-2.27\left(\mathrm{~m}, 14 \mathrm{H}, \mathrm{CH}_{3}\right.$ and $\left.\mathrm{CH}_{2}\right) ;{ }^{13} \mathrm{C} \mathrm{NMR}(75$ $\left.\mathrm{MHz}, \mathrm{CDCl}_{3}\right) \delta 150.6,143.9,141.4,138.7,136.5,132.8,132.5,131.9,129.3,127.6,127.4$, 127.3, 127.2, 125.9, 118.9, 97.3, 58.9, 55.2, 38.4, 30.2, 20.3; HRMS (EI) Calcd for $\mathrm{C}_{37} \mathrm{H}_{40} \mathrm{O}_{4}$ : 548.2926; Found 548.2931.

\section{Synthesis of $(R)-6,6$ '-dimethyl-1,1'-spirobiindane-7,7'-diol ((R)-9b)}

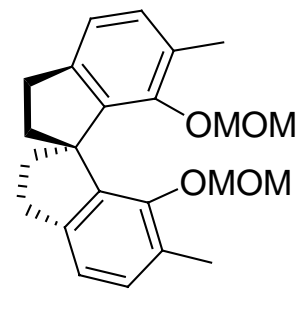

$(R)-\mathbf{8 b}$

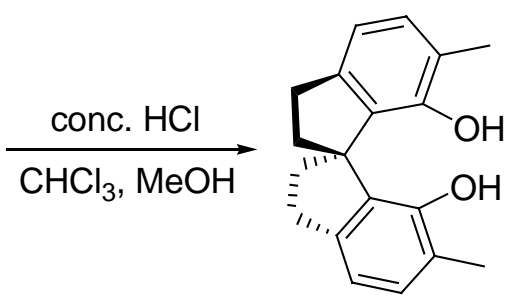

(R)-9b

To a solution of $(R)-8 \mathbf{b}(404 \mathrm{mg}, 1.1 \mathrm{mmol})$ in $8 \mathrm{~mL} \mathrm{CHCl}_{3}$ and $12 \mathrm{~mL} \mathrm{MeOH}$, conc. $\mathrm{HCl}$ $(8.0 \mathrm{~mL})$ was added and the mixture was heated at reflux for $3 \mathrm{~h}$. After cooled to ambient temperature, the mixture was poured into water, extracted by $\mathrm{CH}_{2} \mathrm{Cl}_{2}$ and the combined organic phase was washed with saturated $\mathrm{NaHCO}_{3}$ and brine, dried over $\mathrm{MgSO}_{4}$. The solvent was removed, and the residue was purified by chromatography (ethyl acetate/petroleum ether: $\mathrm{v} / \mathrm{v}=1: 10)$ to give $(R)-9 \mathbf{b}(292 \mathrm{mg}, 95 \%$ yield $)$ as a white solid. Mp $105-106{ }^{\circ} \mathrm{C},[\alpha]^{20}{ }_{\mathrm{D}}+62$ (c 1.0, $\left.\mathrm{CH}_{2} \mathrm{Cl}_{2}\right) ;{ }^{1} \mathrm{H}$ NMR (300 MHz, $\left.\mathrm{CDCl}_{3}\right) \delta 7.03(\mathrm{~d}, J=7.5 \mathrm{~Hz}, 2 \mathrm{H}, \mathrm{Ar}-\mathrm{H}), 6.78$ (d, $J=$ $7.5 \mathrm{~Hz}, 2 \mathrm{H}, \mathrm{Ar}-\mathrm{H}), 4.66(\mathrm{~s}, 2 \mathrm{H}, \mathrm{OH}), 3.02-2.90\left(\mathrm{~m}, 4 \mathrm{H}, \mathrm{CH}_{2}\right), 2.33-1.53\left(\mathrm{~m}, 10 \mathrm{H}, \mathrm{CH}_{3}\right.$ and $\left.\mathrm{CH}_{2}\right) ;{ }^{13} \mathrm{C} \mathrm{NMR}\left(75 \mathrm{MHz}, \mathrm{CDCl}_{3}\right) \delta 151.1,143.1,131.4,129.7,123.0,117.0,57.4,37.7,30.9$, 
15.2; HRMS (EI) Calcd for $\mathrm{C}_{19} \mathrm{H}_{20} \mathrm{O}_{2}$ : 280.1463; Found 280.1462.

\section{Synthesis of $(R)-6,6^{\prime}$-diphenyl-1,1'-spirobiindane-7,7'-diol $((R)-9 \mathrm{c})$}

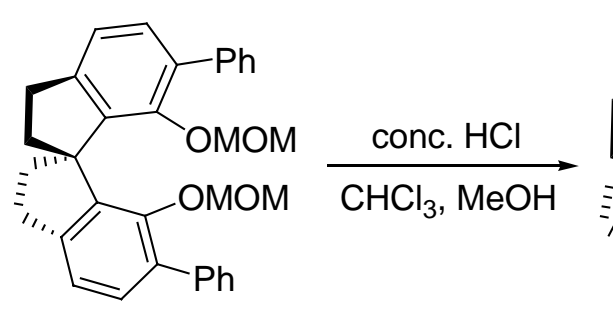

$(R)-8 \mathrm{C}$

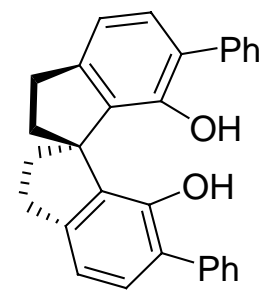

$(R)-9 \mathrm{c}$

Compound (R)-9c was prepared by the same procedure as that for $(R)-9 \mathbf{b}$ (93\% yield). Mp 207-208 ${ }^{\circ} \mathrm{C} .[\alpha]^{20}{ }_{\mathrm{D}}+273\left(\mathrm{c} 1.0, \mathrm{CH}_{2} \mathrm{Cl}_{2}\right) ;{ }^{1} \mathrm{H}$ NMR $\left(300 \mathrm{MHz}, \mathrm{CDCl}_{3}\right) \delta 7.46(\mathrm{~d}, J=6.0 \mathrm{~Hz}$, 4H, Ar-H), 7.42-7.37 (m, 4H, Ar-H), 7.31-7.28 (m, 2H, Ar-H), 7.18 (d, J = 5.7 Hz, 2H, Ar-H), 7.04 (d, J=5.7 Hz, 2H, Ar-H), 5.05 (s, 2H, OH), 3.10-3.02 (m, 4H, $\left.\mathrm{CH}_{2}\right), 2.46-2.33$ $\left(\mathrm{m}, 4 \mathrm{H}, \mathrm{CH}_{2}\right) ;{ }^{13} \mathrm{C} \mathrm{NMR}\left(75 \mathrm{MHz}, \mathrm{CDCl}_{3}\right) \delta 149.5,145.2,137.5,132.1,130.5,129.3,128.6$, 127.2, 127.0, 117.4, 58.5, 37.8, 31.2; HRMS (EI) Calcd for $\mathrm{C}_{29} \mathrm{H}_{24} \mathrm{O}_{2}$ : 404.1776; Found 404.1779 .

\section{Synthesis of (R)-6,6'-di(3,5-dimethylphenyl)-1,1'-spirobiindane-7,7'-diol ((R)-9d)}

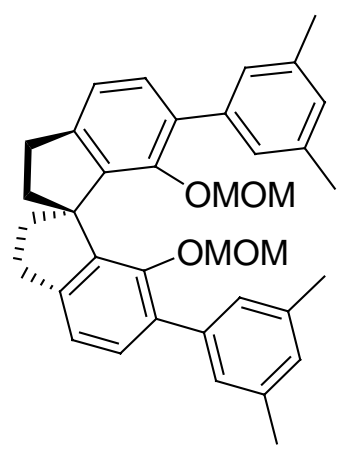

$(R)-8 \mathbf{d}$

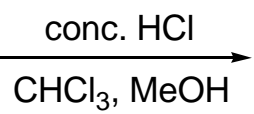

$(R)-9 d$

Compound $(R)-9 d$ was prepared by the same procedure as that for $(R)-9 \mathbf{b}(97 \%$ yield) as a foamy solid. $[\alpha]^{20}{ }_{\mathrm{D}}+127\left(c \mathrm{c} 0.5, \mathrm{CH}_{2} \mathrm{Cl}_{2}\right) ;{ }^{1} \mathrm{H}$ NMR $\left(300 \mathrm{MHz}, \mathrm{CDCl}_{3}\right) \delta 7.14(\mathrm{~d}, J=5.7 \mathrm{~Hz}$, 2H, Ar-H), 7.06 (s, 4H, Ar-H), 6.93 (s, 2H, Ar-H), 6.90 (d, J=5.7 Hz, 2H, Ar-H), 5.13 (s, 2H, $\mathrm{OH}), 3.08-3.04\left(\mathrm{~m}, 4 \mathrm{H}, \mathrm{CH}_{2}\right), 2.45-2.31\left(\mathrm{~m}, 16 \mathrm{H}, \mathrm{CH}_{3}\right.$ and $\left.\mathrm{CH}_{2}\right) ;{ }^{13} \mathrm{C} \mathrm{NMR}(75 \mathrm{MHz}$, $\left.\mathrm{CDCl}_{3}\right) \delta 149.4,145.0,138.3,137.4,132.4,130.2,128.9,127.0,117.2,58.6,37.9,31.2,21.3$; HRMS (EI) Calcd for $\mathrm{C}_{33} \mathrm{H}_{32} \mathrm{O}_{2}: 460.2402$; Found 460.2390.

Synthesis of $\quad N$-dimethyl-[(R)-6,6'-dimethyl-1,1'-spirobiindane-7,7'-diyl]-phosphoramidite ((R)-4b) 


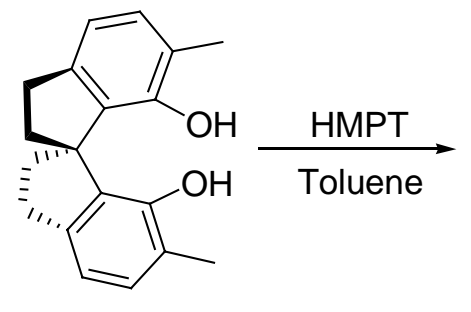

(R)-9b

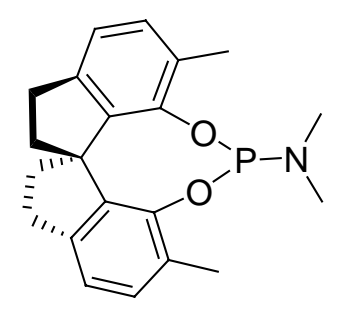

(R)-4b

A mixture of $(R)-9 b(280 \mathrm{mg}, 1.0 \mathrm{mmol})$ and HMPT $(0.22 \mathrm{~mL}, 1.3 \mathrm{mmol})$ in $5 \mathrm{~mL}$ dry toluene was heated at reflux under argon for $6 \mathrm{~h}$. After cooling to room temperature, the mixture was concentrated and purified by chromatography (ethyl acetate/petroleum ether: $\mathrm{v} / \mathrm{v}$ $=1: 15)$ to give $(R)-\mathbf{4 b}(324 \mathrm{mg}, 92 \%$ yield $)$ as a white solid. Mp $185-188{ }^{\circ} \mathrm{C},[\alpha]^{20}{ }_{\mathrm{D}}+289(\mathrm{C}$ $\left.0.5, \mathrm{CH}_{2} \mathrm{Cl}_{2}\right) ;{ }^{1} \mathrm{H}$ NMR $\left(300 \mathrm{MHz}, \mathrm{CDCl}_{3}\right) \delta$ 7.05-6.98 (m, 2H, Ar-H), 6.92-6.87 (m, 2H, Ar-H), 3.04-2.93 (m, 2H, CH$), 2.79-2.70\left(\mathrm{~m}, 2 \mathrm{H}, \mathrm{CH}_{2}\right), 2.33-2.05\left(\mathrm{~m}, 14 \mathrm{H}, \mathrm{CH}_{3}\right.$ and $\left.\mathrm{CH}_{2}\right)$, $1.95-1.84\left(\mathrm{~m}, 2 \mathrm{H}, \mathrm{CH}_{2}\right) ;{ }^{31} \mathrm{P} \mathrm{NMR}\left(121 \mathrm{MHz}, \mathrm{CDCl}_{3}\right) \delta 121.5 ;{ }^{13} \mathrm{C} \mathrm{NMR}\left(75 \mathrm{MHz}, \mathrm{CDCl}_{3}\right) \delta$ $146.6,146.5,144.8,144.7,143.0,142.2,142.1,140.9$, 129.7, 129.6, 128.8, 128.7, 120.6, $119.9,59.2,38.5,38.3,35.7,35.4,30.3,30.0,29.6,16.4,16.3$, 16.0; HRMS (EI) Calcd for $\mathrm{C}_{21} \mathrm{H}_{24} \mathrm{O}_{2} \mathrm{NP}$ : 353.1544; Found 353.1540.

$\begin{array}{lll}\text { Synthesis } & \text { of } & N \text {-dimethyl-[(R)-6,6'-diphenyl-1, } 1^{\prime} \text {-spirobiindane-7,7'-diyl]- } \\ \text { phosphoramidite }((R)-4 c)\end{array}$

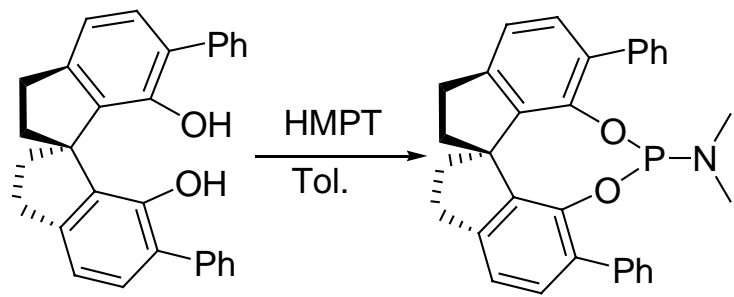

$(R)-9 \mathrm{c}$

$(R)-4 c$

Compound $(R)-\mathbf{4 c}$ was prepared by the same procedure as that for $(R)-\mathbf{4 b}(77 \%$ yield) as a white solid. Mp 258-259 ${ }^{\circ} \mathrm{C},[\alpha]^{20}{ }_{\mathrm{D}}+194\left(\mathrm{c} 0.32, \mathrm{CH}_{2} \mathrm{Cl}_{2}\right) ;{ }^{1} \mathrm{H}$ NMR $\left(300 \mathrm{MHz}, \mathrm{CDCl}_{3}\right) \delta$ $7.53(\mathrm{~d}, J=7.5 \mathrm{~Hz}, 2 \mathrm{H}, \mathrm{Ar}-\mathrm{H}), 7.42$ (d, $J=7.2 \mathrm{~Hz}, 2 \mathrm{H}, \mathrm{Ar}-\mathrm{H}), 7.24-6.98$ (m, 10H, Ar-H), 3.09-2.77 (m, 4H, $\left.\mathrm{CH}_{2}\right), 2.30-1.99\left(\mathrm{~m}, 4 \mathrm{H}, \mathrm{CH}_{2}\right), 1.63\left(\mathrm{~d}, J=9.0 \mathrm{~Hz}, 6 \mathrm{H}, \mathrm{CH}_{3}\right) ;{ }^{31} \mathrm{P}$ NMR $\left(121 \mathrm{MHz}, \mathrm{CDCl}_{3}\right) \delta 123.3 ;{ }^{13} \mathrm{C} \mathrm{NMR}\left(75 \mathrm{MHz}, \mathrm{CDCl}_{3}\right) \delta 144.5,144.4,144.0,143.4,142.7$, $142.6,142.3,142.2,141.2,137.7,137.2,133.2,133.1,132.6,128.8,128.6,128.4,128.3$, 126.8, 126.7, 125.6, 125.4, 120.1, 119.5, 58.6, 37.5, 33.3, 33.0, 29.6, 29.2; HRMS (EI) Calcd for $\mathrm{C}_{31} \mathrm{H}_{28} \mathrm{O}_{2} \mathrm{NP}: 477.1857$; Found 477.1859.

Synthesis of $\quad N$-dimethyl- $\left[(R)-6,6^{\prime}\right.$-di(3,5-dimethylphenyl)-1,1'-spirobiindane-7,7'diyl]-phosphoramidite $((R)-4 d)$ 


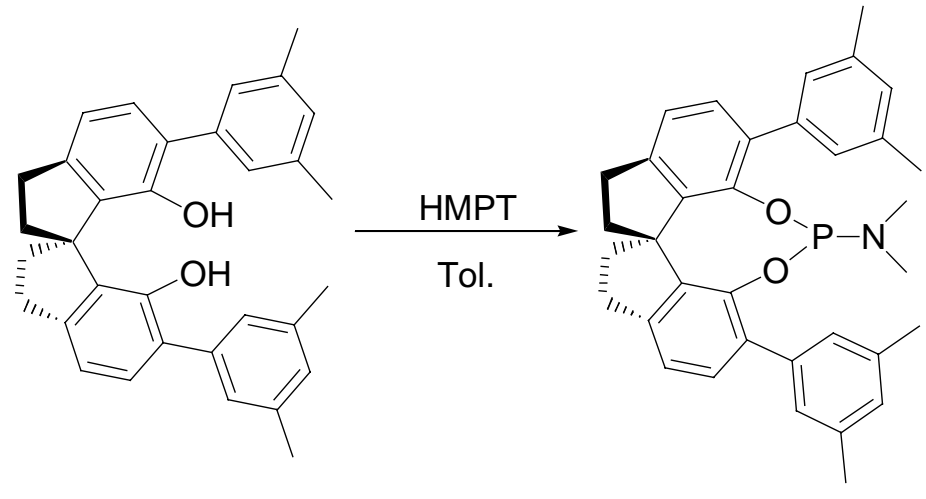

$(R)-9 d$

$(R)-\mathbf{4 d}$

Compound $(R)-\mathbf{4 d}$ was prepared by the same procedure as that for $(R)-\mathbf{4 b}(70 \%$ yield) as a foamy solid. $[\alpha]^{20}{ }_{\mathrm{D}}+173\left(\mathrm{c} 0.32, \mathrm{CH}_{2} \mathrm{Cl}_{2}\right) ;{ }^{1} \mathrm{H} \mathrm{NMR}\left(300 \mathrm{MHz}, \mathrm{CDCl}_{3}\right) \delta 7.28(\mathrm{~d}, J=5.7 \mathrm{~Hz}$, 2H, Ar-H), 7.24 (d, J = 9.3 Hz, 2H, Ar-H), 7.17 (s, 2H, Ar-H), 7.08-7.04 (m, 2H, Ar-H), 6.84 (d, $J=6.6 \mathrm{~Hz}, 2 \mathrm{H}, \mathrm{Ar}-\mathrm{H}), 3.13-3.04\left(\mathrm{~m}, 2 \mathrm{H}, \mathrm{CH}_{2}\right), 2.91-2.83\left(\mathrm{~m}, 2 \mathrm{H}, \mathrm{CH}_{2}\right), 2.34-2.27(\mathrm{~m}$, $14 \mathrm{H}, \mathrm{CH}_{2}$ and $\left.\mathrm{CH}_{3}\right), 2.20-2.08\left(\mathrm{~m}, 2 \mathrm{H}, \mathrm{CH}_{2}\right), 1.75\left(\mathrm{~d}, J=6.6 \mathrm{~Hz}, 6 \mathrm{H}, \mathrm{CH}_{3}\right) ;{ }^{31} \mathrm{P} \mathrm{NMR}(121$ $\left.\mathrm{MHz}, \mathrm{CDCl}_{3}\right) \delta 122.8 ;{ }^{13} \mathrm{C} \mathrm{NMR}\left(75 \mathrm{MHz}, \mathrm{CDCl}_{3}\right) \delta 145.5,145.4,144.6,144.2,143.7,143.6$, $143.3,143.2,142.0,138.7,137.9,137.0,133.9$, 129.4, 129.3, 128.2, 127.9, 127.6, 127.2, 121.1, 120.3, 59.7, 38.6, 38.4, 34.4, 34.1, 30.6, 30.2, 21.2; HRMS (EI) calcd for $\mathrm{C}_{35} \mathrm{H}_{36} \mathrm{O}_{2} \mathrm{NP}$ : 533.2483; Found 533.2480.

Synthesis of $N$-diethyl-[(R)-6,6'-diphenyl-1,1'-spirobiindane-7,7'-diyl]- phosphoramidite $((R)-4 e)$

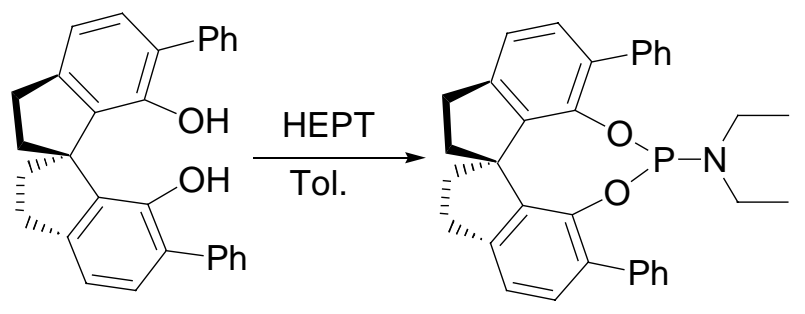

(R)-9c

$(R)-4 \mathrm{e}$

Compound $(R)-4 \mathbf{e}$ was prepared in $63 \%$ yield by the same procedure as that for $(R)-4 \mathbf{b}$ using hexaethylphosphorous triamide as a foamy solid. $[\alpha]^{20}{ }_{\mathrm{D}}+324\left(\mathrm{c} 0.5, \mathrm{CH}_{2} \mathrm{Cl}_{2}\right) ;{ }^{1} \mathrm{H}$ NMR (300 $\left.\mathrm{MHz}, \mathrm{CDCl}_{3}\right) \delta$ 7.50-7.40 (m, 4H, Ar-H), 7.21-6.94 (m, 10H, Ar-H), 3.06-2.74 (m, 4H, $\left.\mathrm{CH}_{2}\right), 2.27-1.78\left(\mathrm{~m}, 8 \mathrm{H}, \mathrm{CH}_{2}\right), 0.47\left(\mathrm{t}, J=6.9 \mathrm{~Hz}, 6 \mathrm{H}, \mathrm{CH}_{3}\right) ;{ }^{31} \mathrm{P} \mathrm{NMR}\left(121 \mathrm{MHz}, \mathrm{CDCl}_{3}\right) \delta$ $125.7 ;{ }^{13} \mathrm{C}$ NMR $\left(75 \mathrm{MHz}, \mathrm{CDCl}_{3}\right) \delta 146.1,146.0,145.0,144.5,144.0,143.9,143.3,143.2$, $141.9,139.0,138.3,134.5,134.4,133.7,130.1,129.6,129.3,127.8,127.7,126.6,126.4$, 121.2, 120.4, 59.7, 40.7, 40.4, 38.7, 38.5, 30.7, 30.3, 16.4, 16.3; HRMS (EI) Calcd for $\mathrm{C}_{33} \mathrm{H}_{32} \mathrm{O}_{2} \mathrm{NP}:$ 505.2170; Found 505.2178.

Synthesis of $(R)-6,6$ '-diphenyl-SIPHOS-morpholine $((R)-4 f)$ 


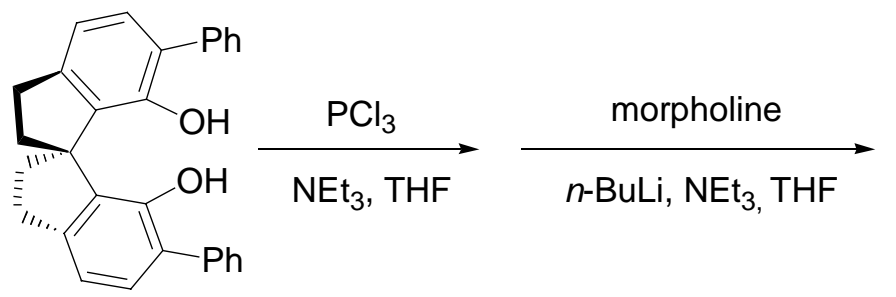

(R)-9c

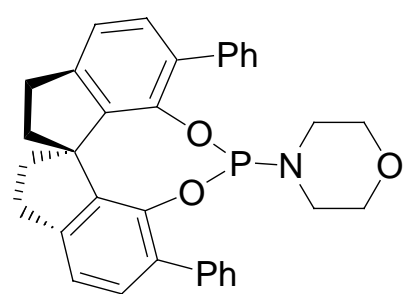

$(R)-\mathbf{4 f}$

To a chilled solution of $\mathrm{PCl}_{3}(56 \mu \mathrm{L}, 0.62 \mathrm{mmol}), \mathrm{Et}_{3} \mathrm{~N}(170 \mu \mathrm{L}, 1.24 \mathrm{mmol})$ in THF $(15 \mathrm{~mL})$, a solution of (R)-6,6'-diphenyl-1,1'-spirobiindane-7,7'-diol $(250 \mathrm{mg}, 0.62 \mathrm{mmol})$ in $5 \mathrm{~mL}$ THF was added at $-78{ }^{\circ} \mathrm{C}$. The reaction mixture was stirred for $3 \mathrm{~h}$, and warmed to room temperature and filtered under nitrogen. The filtrate was cooled to $-78{ }^{\circ} \mathrm{C}$ and treated with lithium morpholinate prepared from morpholine $(81 \mu \mathrm{L}, 0.93 \mathrm{mmol})$ and $n$-butyllithium $(2.2$ $\mathrm{M}$ solution in hexane, $0.42 \mathrm{~mL}, 0.93 \mathrm{mmol}$ ) in $5 \mathrm{~mL}$ THF at $-30{ }^{\circ} \mathrm{C}$. The resulted solution was warmed to room temperature and stirred overnight. The solvent was removed in vacuum and the residue was passed through a silica gel plug with ethyl acetate/petroleum ether $(1: 40)$ to afford pure product $(R)-\mathbf{4 f}\left(62 \%\right.$ yield) as a white solid. Mp 267-269 ${ }^{\circ} \mathrm{C},[\alpha]^{20}{ }_{\mathrm{D}}+327(\mathrm{c} 0.5$, $\mathrm{CH}_{2} \mathrm{Cl}_{2}$ ); ${ }^{1} \mathrm{H}$ NMR (300 MHz, $\left.\mathrm{CDCl}_{3}\right) \delta 7.62(\mathrm{~d}, J=7.2 \mathrm{~Hz}, 2 \mathrm{H}, \mathrm{Ar}-\mathrm{H}), 7.52(\mathrm{~d}, J=7.2 \mathrm{~Hz}$, 2H, Ar-H), 7.36-7.07 (m, 10H, Ar-H), 3.23-3.04 (m, 6H, $\mathrm{CH}_{2}$ ), 2.94-2.84 (m, 2H, $\mathrm{CH}_{2}$ ), 2.38-2.27 (m, 2H, $\left.\mathrm{CH}_{2}\right), 2.18-2.04\left(\mathrm{~m}, 6 \mathrm{H}, \mathrm{CH}_{2}\right) ;{ }^{31} \mathrm{P} \mathrm{NMR}\left(121 \mathrm{MHz}, \mathrm{CDCl}_{3}\right) \delta 121.1 ;{ }^{13} \mathrm{C}$ NMR $\left(75 \mathrm{MHz}, \mathrm{CDCl}_{3}\right) \delta 145.3,145.2,145.1,144.5,143.3,143.2,143.2,142.0,138.7$, $138.1,134.3,134.2,133.5,130.1,129.4,129.3,127.8,126.8,126.5,121.4,120.7,67.9,59.6$, 43.3, 43.0, 38.7, 38.4, 31.8, 30.7, 30.2, 22.6, 14.0; HRMS (EI) Calcd for $\mathrm{C}_{33} \mathrm{H}_{30} \mathrm{O}_{3} \mathrm{NP}$ : 519.1963; Found 519.1955.

\section{(2) General Procedure for Asymmetric Reductive Coupling Reaction}

A mixture of $\mathrm{Ni}(\mathrm{acac})_{2}(2.6 \mathrm{mg}, 0.01 \mathrm{mmol})$, ligand $(S)-4 \mathrm{f}(6.2 \mathrm{mg}, 0.012 \mathrm{mmol})$, 1,4-diphenylbuta-1,3-diene 1 (41.2 $\mathrm{mg}, 0.2 \mathrm{mmol}$ ), benzaldehyde $\mathbf{2 a}$ (42.4 $\mathrm{mg}, 0.4 \mathrm{mmol}$ ) and $2.0 \mathrm{~mL}$ toluene in a Schlenk tube was stirred at room temperature for $5 \mathrm{~min}$. A solution of $\mathrm{ZnEt}_{2}$ (0.48 mmol, $2.4 \mathrm{M}$ in tol.) was added slowly. The resulted residue was stirred at ambient temperature for $1 \mathrm{~h}$ and then quenched by $1 \mathrm{~N} \mathrm{HCl}(0.5 \mathrm{~mL})$. The mixture was extracted with ethyl acetate, and the extract was washed with saturated $\mathrm{NaHCO}_{3}$ and brine, dried over $\mathrm{MgSO}_{4}$ and concentrated in vacuum. The residue was purified by column chromatography on silica gel with ethyl acetate/petroleum ether $(\mathrm{v} / \mathrm{v}=1: 8)$ to give $\mathbf{3 a}$ in $99 \%$ yield.

\section{(3) Analytical Data of Bishomoallylic Alcohols 3}

(E)-1,2,5-Triphenylpent-4-en-1-ol (3a) 


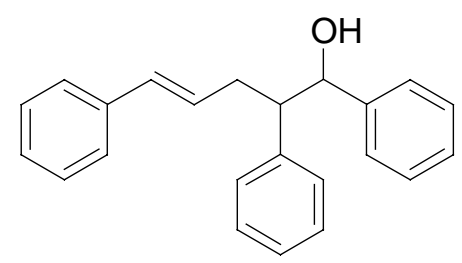

Oil and solidified by standing. $[\alpha]^{20}{ }_{\mathrm{D}}-9.8\left(\mathrm{c} 0.5, \mathrm{CH}_{2} \mathrm{Cl}_{2}\right), 96 \%$ ee, $>99: 1 d r$. HPLC condition: Chiralpak AD-H column, $n$-hexane/2-propanol = 95:5, $1.0 \mathrm{~mL} / \mathrm{min}, 254 \mathrm{~nm} \mathrm{UV}$ detector, $t_{1}=18.3 \mathrm{~min}$ and $t_{2}=20.4 \mathrm{~min} .{ }^{1} \mathrm{H} \mathrm{NMR}\left(\mathrm{CDCl}_{3}, 300 \mathrm{MHz}\right): \delta 7.22-7.06(\mathrm{~m}, 15 \mathrm{H}$, Ar-H), 6.33 (d, $J=15.9 \mathrm{~Hz}, 1 \mathrm{H}, \mathrm{CH}), 6.04-5.94(\mathrm{~m}, 1 \mathrm{H}, \mathrm{CH}), 4.86(\mathrm{dd}, J=6.6$ and $3.3 \mathrm{~Hz}$, $1 \mathrm{H}, \mathrm{CH}), 3.14-3.07(\mathrm{~m}, 1 \mathrm{H}, \mathrm{CH}), 2.84-2.64\left(\mathrm{~m}, 2 \mathrm{H}, \mathrm{CH}_{2}\right), 2.02-1.98(\mathrm{~m}, 1 \mathrm{H}, \mathrm{OH}),{ }^{13} \mathrm{C}$ NMR $\left(\mathrm{CDCl}_{3}, 75 \mathrm{MHz}\right): \delta 142.7,141.1,137.7,131.4,128.9,128.8,128.4,128.2,128.0$, 127.4, 126.9, 126.5, 126.0, 78.1, 53.7, 33.7; MS (EI) m/z 314.0; Anal. Calcd for $\mathrm{C}_{23} \mathrm{H}_{22} \mathrm{O}$ : C 87.86, H 7.05; Found: C 87.74, H 6.83.

\section{(E)-1-(2-Methylphenyl)-2,5-diphenylpent-4-en-1-ol (3b)}

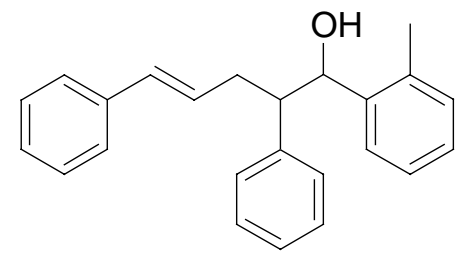

Colorless oil. $[\alpha]^{20}{ }_{\mathrm{D}}-55.1$ (c 1.07, $\mathrm{CH}_{2} \mathrm{Cl}_{2}$ ), 93\% ee, $>99: 1 \mathrm{dr}$. HPLC condition: Chiralpak AD-H column, $n$-hexane/2-propanol $=98: 2,0.8 \mathrm{~mL} / \mathrm{min}, 254 \mathrm{~nm}$ UV detector, $t_{1}=63.8 \mathrm{~min}$ and $t_{2}=81.0 \mathrm{~min} .{ }^{1} \mathrm{H} \mathrm{NMR}\left(\mathrm{CDCl}_{3}, 300 \mathrm{MHz}\right): \delta 7.53(\mathrm{~d}, J=7.8 \mathrm{~Hz}, 1 \mathrm{H}, \mathrm{Ar}-\mathrm{H}), 7.25-7.02$ $(\mathrm{m}, 13 \mathrm{H}, \mathrm{Ar}-\mathrm{H}), 6.32(\mathrm{~d}, J=15.9 \mathrm{~Hz}, 1 \mathrm{H}, \mathrm{CH}), 6.00-5.91(\mathrm{~m}, 1 \mathrm{H}, \mathrm{CH}), 5.09(\mathrm{dd}, 1 \mathrm{H}, J=6.0$ and $3.6 \mathrm{~Hz}, \mathrm{CH}), 3.14-3.07(\mathrm{~m}, 1 \mathrm{H}, \mathrm{CH}), 2.92-2.80\left(\mathrm{~m}, 2 \mathrm{H}, \mathrm{CH}_{2}\right), 2.18\left(\mathrm{~s}, 3 \mathrm{H}, \mathrm{CH}_{3}\right)$, $1.84-1.83(\mathrm{~m}, 1 \mathrm{H}, \mathrm{OH}),{ }^{13} \mathrm{C} \mathrm{NMR}\left(\mathrm{CDCl}_{3}, 75 \mathrm{MHz}\right): \delta 141.6,141.2,137.7,134.7,131.2$, $130.3,129.0,128.6,128.3,128.2$, 127.2, 126.8, 126.6, 126.3, 125.9, 74.5, 52.2, 32.6, 19.2; MS (EI) m/z 328.1; Anal. Calcd for $\mathrm{C}_{24} \mathrm{H}_{24} \mathrm{O}$ : C 87.76, H 7.37; Found: C 87.53, H 7.05.

(E)-1-(2-Methoxyphenyl)-2,5-diphenylpent-4-en-1-ol (3c)<smiles>COc1ccccc1C(O)C(C/C=C/c1ccccc1)c1ccccc1</smiles>

Colorless oil. $[\alpha]^{20}{ }_{D}-13.9$ (c 1.13, $\mathrm{CH}_{2} \mathrm{Cl}_{2}$ ), 91\% ee, $>99: 1 \mathrm{dr}$. HPLC condition: Chiralpak AD-H column, $n$-hexane/2-propanol $=90: 10,1.0 \mathrm{~mL} / \mathrm{min}, 254 \mathrm{~nm} \mathrm{UV} \mathrm{detector,} t_{1}=25.6 \mathrm{~min}$ and $t_{2}=30.4 \mathrm{~min} .{ }^{1} \mathrm{H}$ NMR $\left(\mathrm{CDCl}_{3}, 300 \mathrm{MHz}\right): \delta 7.18-7.07(\mathrm{~m}, 12 \mathrm{H}, \mathrm{Ar}-\mathrm{H}), 6.81(\mathrm{t}, J=8.4$ $\mathrm{Hz}, 2 \mathrm{H}, \mathrm{Ar}-\mathrm{H}), 6.32$ (d, J=15.9 Hz, 1H, CH), 6.06-5.96 (m, 1H, CH), 5.00 (t, $J=6.9 \mathrm{~Hz}$, 
$1 \mathrm{H}, \mathrm{CH}), 3.78\left(\mathrm{~s}, 3 \mathrm{H}, \mathrm{CH}_{3}\right), 3.30-3.22(\mathrm{~m}, 1 \mathrm{H}, \mathrm{CH}), 2.91-2.68\left(\mathrm{~m}, 2 \mathrm{H}, \mathrm{CH}_{2}\right), 2.74(\mathrm{~d}, J=6.6$ $\mathrm{Hz}, 1 \mathrm{H}, \mathrm{OH}),{ }^{13} \mathrm{C} \mathrm{NMR}\left(\mathrm{CDCl}_{3}, 75 \mathrm{MHz}\right): \delta 156.4,142.0,137.9,131.0,130.4,129.3,128.6$, $128.3,128.2,128.0,126.7,126.3,125.9,120.4,110.4,75.7,55.2,52.0,33.6 ; \mathrm{MS}$ (EI) $\mathrm{m} / \mathrm{z}$ 344.1; Anal. Calcd for $\mathrm{C}_{24} \mathrm{H}_{24} \mathrm{O}$ : C 83.69, H 7.02; Found: C 83.63, H 6.99.

(E)-2,5-Diphenyl-1-m-tolylpent-4-en-1-ol (3d)<smiles>Cc1cccc(C(O)C(C/C=C/c2ccccc2)c2ccccc2)c1</smiles>

Colorless oil. $[\alpha]^{20}{ }_{\mathrm{D}}-11.4\left(c 0.75, \mathrm{CH}_{2} \mathrm{Cl}_{2}\right), 94 \%$ ee, $>99: 1 d r$. HPLC condition: Chiralcel OD-H column, $n$-hexane/2-propanol $=95: 5,1.0 \mathrm{~mL} / \mathrm{min}, 254 \mathrm{~nm} \mathrm{UV} \mathrm{detector,} t_{1}=17.3 \mathrm{~min}$ and $t_{2}=21.3 \mathrm{~min} .{ }^{1} \mathrm{H} \mathrm{NMR}\left(\mathrm{CDCl}_{3}, 300 \mathrm{MHz}\right): \delta 7.52(\mathrm{~d}, 1 \mathrm{H}, \mathrm{J}=7.8 \mathrm{~Hz}, \mathrm{Ar}-\mathrm{H}), 7.21-7.02$ $(\mathrm{m}, 13 \mathrm{H}, \mathrm{Ar}-\mathrm{H}), 6.30$ (d, $J=15.9 \mathrm{~Hz}, 1 \mathrm{H}, \mathrm{CH}), 6.00-5.90(\mathrm{~m}, 1 \mathrm{H}, \mathrm{CH}), 5.07$ (dd, $1 \mathrm{H}, J=6.0$ and $3.3 \mathrm{~Hz}, \mathrm{CH}), 3.14-3.07(\mathrm{~m}, 1 \mathrm{H}, \mathrm{CH}), 2.87-2.79\left(\mathrm{~m}, 2 \mathrm{H}, \mathrm{CH}_{2}\right), 2.18\left(\mathrm{~s}, 3 \mathrm{H}, \mathrm{CH}_{3}\right), 1.84(\mathrm{~d}$, $J=3.3 \mathrm{~Hz}, 1 \mathrm{H}, \mathrm{OH}),{ }^{13} \mathrm{C} \mathrm{NMR}\left(\mathrm{CDCl}_{3}, 75 \mathrm{MHz}\right): \delta 141.6,141.2,137.7,134.7,131.2,130.3$, 129.0, 128.6, 128.3, 127.2, 126.8, 126.6, 126.3, 125.9, 74.5, 52.1, 32.6, 19.2; HRMS (EI) Calcd for $\mathrm{C}_{24} \mathrm{H}_{24} \mathrm{O}: 328.1827$; Found 328.1822.

(E)-1-(3-Methoxyphenyl)-2,5-diphenylpent-4-en-1-ol (3e)<smiles>COc1cccc(C(O)C(C/C=C/c2ccccc2)c2ccccc2)c1</smiles>

Colorless oil. $[\alpha]^{20}{ }_{D}-3.5\left(c \quad 0.75, \mathrm{CH}_{2} \mathrm{Cl}_{2}\right), 92 \%$ ee, $>99: 1 d r$. HPLC condition: Chiralcel OD-H column, $n$-hexane/2-propanol $=95: 5,1.0 \mathrm{~mL} / \mathrm{min}, 254 \mathrm{~nm} \mathrm{UV} \mathrm{detector,} t_{1}=38.3 \mathrm{~min}$ and $t_{2}=52.8 \mathrm{~min} .{ }^{1} \mathrm{H}$ NMR $\left(\mathrm{CDCl}_{3}, 300 \mathrm{MHz}\right): \delta 7.25-7.09(\mathrm{~m}, 11 \mathrm{H}, \mathrm{Ar}-\mathrm{H}), 6.78-6.71(\mathrm{~m}$, $3 \mathrm{H}, \mathrm{Ar}-\mathrm{H}), 6.34(\mathrm{~d}, J=15.9 \mathrm{~Hz}, 1 \mathrm{H}, \mathrm{CH}), 6.05-5.95(\mathrm{~m}, 1 \mathrm{H}, \mathrm{CH}), 4.87(\mathrm{dd}, J=6.6$ and 3.3 $\mathrm{Hz}, 1 \mathrm{H}, \mathrm{CH}), 3.71\left(\mathrm{~s}, 3 \mathrm{H}, \mathrm{CH}_{3}\right), 3.15-3.08(\mathrm{~m}, 1 \mathrm{H}, \mathrm{CH}), 2.86-2.65\left(\mathrm{~m}, 2 \mathrm{H}, \mathrm{CH}_{2}\right), 1.98$ (d, J $=3.3 \mathrm{~Hz}, 1 \mathrm{H}, \mathrm{OH}),{ }^{13} \mathrm{C} \mathrm{NMR}\left(\mathrm{CDCl}_{3}, 75 \mathrm{MHz}\right): \delta 159.3,144.3,141.0,137.7,131.3,129.0$, 128.8, 128.8, 128.3, 128.1, 126.8, 126.5, 125.9, 118.8, 113.1, 111.9, 77.96, 55.1, 53.6, 33.6; HRMS (EI) Calcd for $\mathrm{C}_{24} \mathrm{H}_{24} \mathrm{O}_{2}: 344.1776$; Found 344.1774.

(E)-2,5-Diphenyl-1-p-tolylpent-4-en-1-ol (3f) 


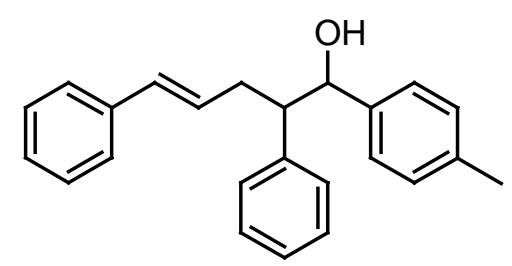

Colorless oil. $[\alpha]^{20}{ }_{D}+4.9\left(c \quad 0.32, \mathrm{CH}_{2} \mathrm{Cl}_{2}\right), 95 \%$ ee, $>99: 1 \mathrm{dr}$. HPLC condition: Chiralpak AD-H column, $n$-hexane/2-propanol $=95: 5,1.0 \mathrm{~mL} / \mathrm{min}, 254 \mathrm{~nm} \mathrm{UV}$ detector, $t_{1}=17.7 \mathrm{~min}$ and $t_{2}=20.4 \mathrm{~min} .{ }^{1} \mathrm{H}$ NMR $\left(\mathrm{CDCl}_{3}, 300 \mathrm{MHz}\right): \delta 7.14-6.93(\mathrm{~m}, 14 \mathrm{H}, \mathrm{Ar}-\mathrm{H}), 6.25(\mathrm{~d}, J=$ $15.9 \mathrm{~Hz}, 1 \mathrm{H}, \mathrm{CH}), 5.96-5.86(\mathrm{~m}, 1 \mathrm{H}, \mathrm{CH}), 4.75(\mathrm{~d}, J=6.3 \mathrm{~Hz}, 1 \mathrm{H}, \mathrm{CH}), 3.05-2.98(\mathrm{~m}, 1 \mathrm{H}$, $\mathrm{CH}), 2.78-2.55\left(\mathrm{~m}, 2 \mathrm{H}, \mathrm{CH}_{2}\right), 2.19\left(\mathrm{~s}, 3 \mathrm{H}, \mathrm{CH}_{3}\right), 1.90(\mathrm{~s}, 1 \mathrm{H}, \mathrm{OH}) ;{ }^{13} \mathrm{C} \mathrm{NMR}\left(\mathrm{CDCl}_{3}, 75\right.$ MHz): $\delta 141.2,139.7,137.8,137.0,131.3,128.9,128.8,128.4,128.2,126.8,126.5,126.0$, 77.9, 53.7, 33.9, 21.1; MS (EI) m/z 328.1; Anal. Calcd for $\mathrm{C}_{24} \mathrm{H}_{24} \mathrm{O}: \mathrm{C}$ 87.76, H 7.37; Found: C 87.71, H 7.39.

\section{(E)-1-(4-Methoxyphenyl)-2,5-diphenylpent-4-en-1-ol (3g)}<smiles>COc1ccc(C(O)C(C/C=C/c2ccccc2)c2ccccc2)cc1</smiles>

Colorless oil. $[\alpha]^{20}{ }_{D}-17.5$ (c 1.47, $\mathrm{CH}_{2} \mathrm{Cl}_{2}$ ), 96\% ee, >99:1 dr, HPLC condition: Chiralpak AS column, $n$-hexane/2-propanol $=98: 2,0.8 \mathrm{~mL} / \mathrm{min}, 254 \mathrm{~nm} \mathrm{UV}$ detector, $t_{1}=47.9 \mathrm{~min}$ and $t_{2}=70.1 \mathrm{~min} .{ }^{1} \mathrm{H} \mathrm{NMR}\left(\mathrm{CDCl}_{3}, 300 \mathrm{MHz}\right): \delta 7.19-7.04(\mathrm{~m}, 12 \mathrm{H}, \mathrm{Ar}-\mathrm{H}), 6.76-6.73(\mathrm{~m}, 2 \mathrm{H}$, Ar-H), $6.34(\mathrm{~d}, J=15.9 \mathrm{~Hz}, 1 \mathrm{H}, \mathrm{CH}), 6.05-5.95(\mathrm{~m}, 1 \mathrm{H}, \mathrm{CH}), 4.82(\mathrm{dd}, J=6.6$ and $3.0 \mathrm{~Hz}$, $1 \mathrm{H}, \mathrm{CH}), 3.73\left(\mathrm{~s}, 3 \mathrm{H}, \mathrm{CH}_{3}\right), 3.12-3.05(\mathrm{~m}, 1 \mathrm{H}, \mathrm{CH}), 2.88-2.63\left(\mathrm{~m}, 2 \mathrm{H}, \mathrm{CH}_{2}\right), 2.02(\mathrm{~d}, J=3.3$ $\mathrm{Hz}, 1 \mathrm{H}, \mathrm{OH}),{ }^{13} \mathrm{C} \mathrm{NMR}\left(\mathrm{CDCl}_{3}, 75 \mathrm{MHz}\right): \delta 158.9,141.1,137.8,134.9,131.3,128.9,128.4$, 128.2, 127.7, 126.8, 126.5, 126.0, 113.4, 77.6, 55.2, 53.7, 34.1; MS (EI) m/z 344.1; Anal. Calcd for $\mathrm{C}_{24} \mathrm{H}_{24} \mathrm{O}$ : C 83.69, H 7.02; Found: C 83.68, H 6.80.

(E)-1-(4-(Dimethylamino)phenyl)-2,5-diphenylpent-4-en-1-ol (3h)<smiles>CN(C)c1ccc(C(O)C(C/C=C/c2ccccc2)c2ccccc2)cc1</smiles>

Oil and solidified by standing. $[\alpha]^{20}{ }_{\mathrm{D}}-2.2\left(c \quad 0.54, \mathrm{CH}_{2} \mathrm{Cl}_{2}\right), 96 \%$ ee, 99:1 dr. HPLC condition: Chiralpak AD-H column, $n$-hexane/2-propanol $=95: 5,1.0 \mathrm{~mL} / \mathrm{min}, 254 \mathrm{~nm} \mathrm{UV}$ detector, $t_{1}=51.5 \mathrm{~min}$ and $t_{2}=53.0 \mathrm{~min} .{ }^{1} \mathrm{H} \mathrm{NMR}\left(\mathrm{CDCl}_{3}, 300 \mathrm{MHz}\right): \delta 7.19-6.99(\mathrm{~m}, 12 \mathrm{H}$, Ar-H), 6.58 (d, $J=8.7 \mathrm{~Hz}, 2 \mathrm{H}, \mathrm{Ar}-\mathrm{H}), 6.34$ (d, $J=15.9 \mathrm{~Hz}, 1 \mathrm{H}, \mathrm{CH}), 6.06-5.96$ (m, 1H, CH), 
$4.80(\mathrm{~d}, J=6.6 \mathrm{~Hz}, 1 \mathrm{H}, \mathrm{CH}), 3.14-3.07(\mathrm{~m}, 1 \mathrm{H}, \mathrm{CH}), 2.87\left(\mathrm{~s}, 6 \mathrm{H}, \mathrm{CH}_{3}\right), 2.84-2.62(\mathrm{~m}, 2 \mathrm{H}$, $\left.\mathrm{CH}_{2}\right), 1.92$ (br, $\left.1 \mathrm{H}, \mathrm{OH}\right),{ }^{13} \mathrm{C} \mathrm{NMR}\left(\mathrm{CDCl}_{3}, 75 \mathrm{MHz}\right): \delta 149.9,141.5,137.9,131.2,130.6$, 129.2, 128.9, 128.4, 128.1, 127.5, 126.8, 126.3, 125.9, 112.2, 77.7, 53.6, 40.6, 34.2; HRMS (EI) Calcd for $\mathrm{C}_{25} \mathrm{H}_{27} \mathrm{ON}$ : 357.2065; Found 357.2089.

(E)-1-(4-Chlorophenyl)-2,5-diphenylpent-4-en-1-ol (3i)<smiles>OC(c1ccc(Cl)cc1)C(CC=Cc1ccccc1)c1ccccc1</smiles>

Colorless oil. $[\alpha]^{20}{ }_{\mathrm{D}}+1.5\left(\mathrm{c} 0.65, \mathrm{CH}_{2} \mathrm{Cl}_{2}\right), 90 \%$ ee, $>99: 1 \mathrm{dr}$. HPLC condition: Chiralpak OD-H column, $n$-hexane/2-propanol $=95: 5,1.0 \mathrm{~mL} / \mathrm{min}, 254 \mathrm{~nm} \mathrm{UV} \mathrm{detector,} t_{1}=32.0 \mathrm{~min}$ and $t_{2}=35.0 \mathrm{~min} .{ }^{1} \mathrm{H} \mathrm{NMR}\left(\mathrm{CDCl}_{3}, 400 \mathrm{MHz}\right): \delta 7.24-7.17(\mathrm{~m}, 10 \mathrm{H}, \mathrm{Ar}-\mathrm{H}), 7.09(\mathrm{t}, J=6.8$ $\mathrm{Hz}, 4 \mathrm{H}, \mathrm{Ar}-\mathrm{H}), 6.37$ (d, $J=16.0 \mathrm{~Hz}, 1 \mathrm{H}, \mathrm{CH}), 6.05-5.98(\mathrm{~m}, 1 \mathrm{H}, \mathrm{CH}), 4.85$ (dd, $J=6.4$ and $2.8 \mathrm{~Hz}, 1 \mathrm{H}, \mathrm{CH}), 3.09-3.02(\mathrm{~m}, 1 \mathrm{H}, \mathrm{CH}), 2.83-2.63\left(\mathrm{~m}, 2 \mathrm{H}, \mathrm{CH}_{2}\right), 2.08(\mathrm{~d}, J=3.2 \mathrm{~Hz}, 1 \mathrm{H}$, $\mathrm{OH}) ;{ }^{13} \mathrm{C} \mathrm{NMR}\left(\mathrm{CDCl}_{3}, 75 \mathrm{MHz}\right): \delta 141.1,140.6,137.6,133.0,131.5,128.8,128.5,128.4$, 128.3, 128.1, 127.9, 126.9, 126.7, 126.0, 77.4, 53.7, 33.8; HRMS (EI) Calcd for $\mathrm{C}_{23} \mathrm{H}_{21} \mathrm{OCl}$ : 348.1280; Found 348.1286.

\section{(E)-2,5-Diphenyl-1-(4-(trifluoromethyl)phenyl)pent-4-en-1-ol (3j)}<smiles>OC(c1ccc(C(F)(F)F)cc1)C(C/C=C/c1ccccc1)c1ccccc1</smiles>

Colorless oil. $[\alpha]^{20}{ }_{D}-2.0\left(c \quad 0.34, \mathrm{CH}_{2} \mathrm{Cl}_{2}\right.$ ), 85\% ee, $>99: 1 \mathrm{dr}$. HPLC condition: Chiralpak AD-H column, $n$-hexane/2-propanol $=99: 1,1.0 \mathrm{~mL} / \mathrm{min}, 254 \mathrm{~nm} \mathrm{UV}$ detector, $t_{1}=105.5 \mathrm{~min}$ and $t_{2}=112.8 \mathrm{~min} .{ }^{1} \mathrm{H}$ NMR $\left(\mathrm{CDCl}_{3}, 300 \mathrm{MHz}\right): \delta 7.47(\mathrm{~d}, J=8.4 \mathrm{~Hz}, 2 \mathrm{H}, \mathrm{Ar}-\mathrm{H}), 7.27-7.06$ $(\mathrm{m}, 12 \mathrm{H}, \mathrm{Ar}-\mathrm{H}), 6.34(\mathrm{~d}, J=15.9 \mathrm{~Hz}, 1 \mathrm{H}, \mathrm{CH}), 6.01-5.91(\mathrm{~m}, 1 \mathrm{H}, \mathrm{CH}), 4.90(\mathrm{dd}, J=6.3$ and $3.6 \mathrm{~Hz}, 1 \mathrm{H}, \mathrm{CH}), 3.13-3.06(\mathrm{~m}, 1 \mathrm{H}, \mathrm{CH}), 2.81-2.64\left(\mathrm{~m}, 2 \mathrm{H}, \mathrm{CH}_{2}\right), 2.17$ (d, J=3.6 Hz, 1H, $\mathrm{OH}),{ }^{13} \mathrm{C} \mathrm{NMR}\left(\mathrm{CDCl}_{3}, 75 \mathrm{MHz}\right): \delta 146.6,140.5,137.5,131.6,128.8,128.4,128.3,127.0$, 126.9, 125.9, 125.0, 124.9, 77.5, 53.7, 33.5; HRMS (EI) Calcd for $\mathrm{C}_{24} \mathrm{H}_{21} \mathrm{OF}_{3}$ : 382.1544; Found 382.1546.

(E)-1-(Naphthalen-1-yl)-2,5-diphenylpent-4-en-1-ol (3k) 
<smiles>OC(c1ccccc1)C(C/C=C/c1ccccc1)c1cccc2ccccc12</smiles>

Oil and solidified by standing. $[\alpha]^{20}{ }_{\mathrm{D}}-72.4$ (c 0.94, $\mathrm{CH}_{2} \mathrm{Cl}_{2}$ ), 93\% ee, 98:2 dr. HPLC condition: Chiralpak AD-H column, $n$-hexane/2-propanol =90:10, $1.0 \mathrm{~mL} / \mathrm{min}, 254 \mathrm{~nm} \mathrm{UV}$ detector, $t_{1}=21.4 \mathrm{~min}$ and $t_{2}=27.4 \mathrm{~min} .{ }^{1} \mathrm{H} \mathrm{NMR}\left(\mathrm{CDCl}_{3}, 300 \mathrm{MHz}\right): \delta 8.09(\mathrm{~d}, J=8.1 \mathrm{~Hz}$, $1 \mathrm{H}, \operatorname{Ar}-\mathrm{H}), 7.83(\mathrm{~d}, J=7.5 \mathrm{~Hz}, 1 \mathrm{H}, \operatorname{Ar}-\mathrm{H}), 7.72$ (d, $J=8.1 \mathrm{~Hz}, 1 \mathrm{H}, \operatorname{Ar}-\mathrm{H}), 7.60$ (d, $J=7.2 \mathrm{~Hz}$, $1 \mathrm{H}, \mathrm{Ar}-\mathrm{H}), 7.52-7.38(\mathrm{~m}, 3 \mathrm{H}, \mathrm{Ar}-\mathrm{H}), 7.31-7.05(\mathrm{~m}, 10 \mathrm{H}, \mathrm{Ar}-\mathrm{H}), 6.20(\mathrm{~d}, J=15.6 \mathrm{~Hz}, 1 \mathrm{H}$, $\mathrm{CH}), 5.89-5.79(\mathrm{~m}, 1 \mathrm{H}, \mathrm{CH}), 5.61(\mathrm{t}, J=3.6 \mathrm{~Hz}, 1 \mathrm{H}, \mathrm{CH}), 3.39-3.29(\mathrm{~m}, 1 \mathrm{H}, \mathrm{CH}), 2.88-2.64$ $\left(\mathrm{m}, 2 \mathrm{H}, \mathrm{CH}_{2}\right), 2.14(\mathrm{~d}, J=3.3 \mathrm{~Hz}, 1 \mathrm{H}, \mathrm{OH}),{ }^{13} \mathrm{C} \mathrm{NMR}\left(\mathrm{CDCl}_{3}, 75 \mathrm{MHz}\right): \delta 142.3,138.4$, $137.7,133.8,131.2,130.4,129.0,128.5,128.4,128.3,128.0,126.7,126.6,126.0,125.9$, 125.4, 125.1, 124.3, 75.0, 51.8, 32.3; MS (EI) m/z 364.1; Anal. Calcd for $\mathrm{C}_{23} \mathrm{H}_{22} \mathrm{O}: \mathrm{C} 88.97$, H 6.64; Found: C 88.72, H 6.44.

\section{(E)-1-(Naphthalen-2-yl)-2,5-diphenylpent-4-en-1-ol (3I)}

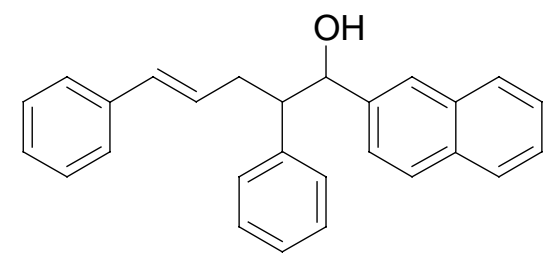

Oil and solidified by standing. $[\alpha]^{20}{ }_{\mathrm{D}}+12.0\left(\mathrm{c} 0.77, \mathrm{CH}_{2} \mathrm{Cl}_{2}\right), 88 \%$ ee, $>99: 1 \mathrm{dr}$. HPLC condition: Chiralpak AD-H column, $n$-hexane/2-propanol =90:10, $1.0 \mathrm{~mL} / \mathrm{min}, 254 \mathrm{~nm} \mathrm{UV}$ detector, $t_{1}=34.2 \mathrm{~min}$ and $t_{2}=37.7 \mathrm{~min} .{ }^{1} \mathrm{H}$ NMR $\left(\mathrm{CDCl}_{3}, 300 \mathrm{MHz}\right): \delta 7.78-7.69(\mathrm{~m}, 3 \mathrm{H}$, Ar-H), 7.44-7.41 (m, 2H, Ar-H), 7.21-7.10 (m, 12H, Ar-H), 6.32 (d, J=15.9 Hz, 1H, CH), 6.04-5.94 (m, 1H, CH), $5.03(\mathrm{dd}, J=6.0$ and $2.7 \mathrm{~Hz}, 1 \mathrm{H}, \mathrm{CH}), 3.25-3.18(\mathrm{~m}, 1 \mathrm{H}, \mathrm{CH})$, 2.86-2.68 (m, 2H, $\left.\mathrm{CH}_{2}\right), 2.15(\mathrm{~d}, J=3.0 \mathrm{~Hz}, 1 \mathrm{H}, \mathrm{OH}),{ }^{13} \mathrm{C} \mathrm{NMR}\left(\mathrm{CDCl}_{3}, 75 \mathrm{MHz}\right): \delta 141.0$, $140.1,137.6,133.1,132.8,131.4,128.8,128.7$, 128.3, 128.2, 127.9, 127.7, 127.6, 126.8, $126.6,126.0,125.9,125.7,125.4,124.6,78.1,53.5,33.5$; HRMS (EI) Calcd for $\mathrm{C}_{27} \mathrm{H}_{24} \mathrm{O}$ : 364.1827; Found 364.1826.

(E)-1-(Furan-2-yl)-2,5-diphenylpent-4-en-1-ol (3m)<smiles>OC(c1ccco1)C(CC=Cc1ccccc1)c1ccccc1</smiles> 
Oil and solidified by standing. $[\alpha]^{20}-31.9\left(c 0.80, \mathrm{CH}_{2} \mathrm{Cl}_{2}\right), 92 \%$ ee, $>99: 1 \mathrm{dr}$. HPLC condition: Chiralpak AD-H column, $n$-hexane/2-propanol =90:10, $1.0 \mathrm{~mL} / \mathrm{min}, 254 \mathrm{~nm} \mathrm{UV}$ detector, $t_{1}=21.4 \mathrm{~min}$ and $t_{2}=22.8 \mathrm{~min} .{ }^{1} \mathrm{H} \mathrm{NMR}\left(\mathrm{CDCl}_{3}, 300 \mathrm{MHz}\right): \delta 7.15-7.01(\mathrm{~m}, 12 \mathrm{H}$, Ar-H), 6.27 (d, $J=15.6 \mathrm{~Hz}, 1 \mathrm{H}, \mathrm{CH}), 6.09$ (dd, $J=3.3$ and $1.8 \mathrm{~Hz}, 1 \mathrm{H}, \mathrm{Ar}-\mathrm{H}), 5.99-5.89$ (m, $1 \mathrm{H}, \mathrm{CH}), 4.76(\mathrm{~d}, J=6.6 \mathrm{~Hz}, 1 \mathrm{H}, \mathrm{CH}), 3.20-3.13(\mathrm{~m}, 1 \mathrm{H}, \mathrm{CH}), 2.82-2.54\left(\mathrm{~m}, 2 \mathrm{H}, \mathrm{CH}_{2}\right), 2.19$ (br, $1 \mathrm{H}, \mathrm{OH}),{ }^{13} \mathrm{C} \mathrm{NMR}\left(\mathrm{CDCl}_{3}, 75 \mathrm{MHz}\right): \delta 155.2,141.6,140.9,137.7,131.7,128.6,128.5$, 128.3, 126.9, 126.8, 126.1 110.2, 107.0, 71.8, 51.4, 34.5; MS (EI) m/z 304.0; Anal. Calcd for $\mathrm{C}_{23} \mathrm{H}_{22} \mathrm{O}$ : C 82.86, H 6.62; Found: C 82.65, H 6.98 .

\section{(E)-2,5-Diphenyl-1-(thiophen-2-yl)pent-4-en-1-ol (3n)}

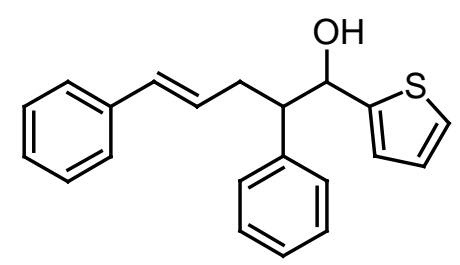

Yellow oil. $[\alpha]_{D}^{20}-1.7$ (c $0.24, \mathrm{CH}_{2} \mathrm{Cl}_{2}$ ), 91\% ee, >99:1 dr. HPLC condition: Chiralpak AD-H column, $n$-hexane/2-propanol $=90: 10,0.6 \mathrm{~mL} / \mathrm{min}, 254 \mathrm{~nm} \mathrm{UV} \mathrm{detector,} t_{1}=38.2 \mathrm{~min}$ and $t_{2}=40.1 \mathrm{~min} .{ }^{1} \mathrm{H} \mathrm{NMR}\left(\mathrm{CDCl}_{3}, 300 \mathrm{MHz}\right): \delta 7.25-7.11(\mathrm{~m}, 11 \mathrm{H}, \mathrm{Ar}-\mathrm{H}), 6.84-6.81(\mathrm{~m}$, $1 \mathrm{H}, \operatorname{Ar}-\mathrm{H}), 6.72(\mathrm{~d}, J=3.6 \mathrm{~Hz}, 1 \mathrm{H}, \mathrm{Ar}-\mathrm{H}), 6.36(\mathrm{~d}, J=15.9 \mathrm{~Hz}, 1 \mathrm{H}, \mathrm{CH}), 6.07-5.97(\mathrm{~m}, 1 \mathrm{H}$, $\mathrm{CH}), 5.12(\mathrm{dd}, J=6.9$ and $3.9 \mathrm{~Hz}, 1 \mathrm{H}, \mathrm{CH}), 3.17-3.10(\mathrm{~m}, 1 \mathrm{H}, \mathrm{CH}), 2.95-2.66\left(\mathrm{~m}, 2 \mathrm{H}, \mathrm{CH}_{2}\right)$, $2.23(\mathrm{~d}, J=4.2 \mathrm{~Hz}, 1 \mathrm{H}, \mathrm{OH}),{ }^{13} \mathrm{C} \mathrm{NMR}\left(\mathrm{CDCl}_{3}, 75 \mathrm{MHz}\right): \delta 146.8,140.8,137.6,131.6$, 128.7, 128.4, 128.2, 126.9, 126.7, 126.4, 126.0, 124.3, 124.2, 74.2, 54.2, 34.5; MS (EI) m/z 320.1; Anal. Calcd for $\mathrm{C}_{23} \mathrm{H}_{22} \mathrm{O}$ : C 78.71, H 6.29; Found: C 78.42, H 6.00.

\section{(E)-5,8-Diphenyloct-7-en-4-ol (3o)}

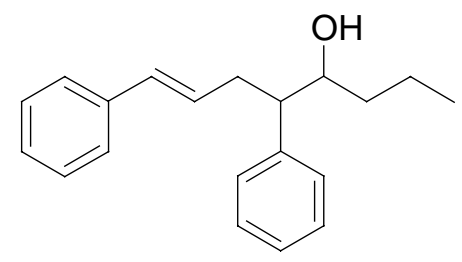

Oil and solidified by standing. $[\alpha]^{20}-15.6\left(c \quad 0.32, \mathrm{CH}_{2} \mathrm{Cl}_{2}\right), 72 \%$ ee, $>99: 1 \mathrm{dr}$. HPLC condition: Chiralpak AD-H column, $n$-hexane/2-propanol =90:10, $1.0 \mathrm{~mL} / \mathrm{min}, 254 \mathrm{~nm} \mathrm{UV}$ detector, $t_{1}=26.0 \mathrm{~min}$ and $t_{2}=28.4 \mathrm{~min} .{ }^{1} \mathrm{H} \mathrm{NMR}\left(\mathrm{CDCl}_{3}, 300 \mathrm{MHz}\right): \delta 7.33-7.17(\mathrm{~m}, 10 \mathrm{H}$, ArH), 6.35 (d, J=15.9 Hz, 1H, CH), 6.09-5.99 (m, 1H, CH), $3.79(\mathrm{~s}, 1 \mathrm{H}, \mathrm{CH}), 2.86-2.75(\mathrm{~m}$, $\left.2 \mathrm{H}, \mathrm{CH}_{2}\right), 2.67-2.60(\mathrm{~m}, 1 \mathrm{H}, \mathrm{CH}), 1.52-1.24\left(\mathrm{~m}, 5 \mathrm{H}, \mathrm{CH}_{2}, \mathrm{OH}\right), 0.85(\mathrm{t}, J=6.6 \mathrm{~Hz}, 3 \mathrm{H}$, $\left.\mathrm{CH}_{3}\right) ;{ }^{13} \mathrm{C} \mathrm{NMR}\left(\mathrm{CDCl}_{3}, 75 \mathrm{MHz}\right): \delta 142.1,137.7,131.2,128.9,128.5,128.4,128.3,126.8$, 126.5, 125.9, 75.1, 52.7, 37.0, 34.8, 19.0, 13.9; HRMS (EI) Calcd for $\mathrm{C}_{20} \mathrm{H}_{24} \mathrm{O}$ : 280.1827; Found 280.1827. 
(4) NMR Spectra for New Ligands
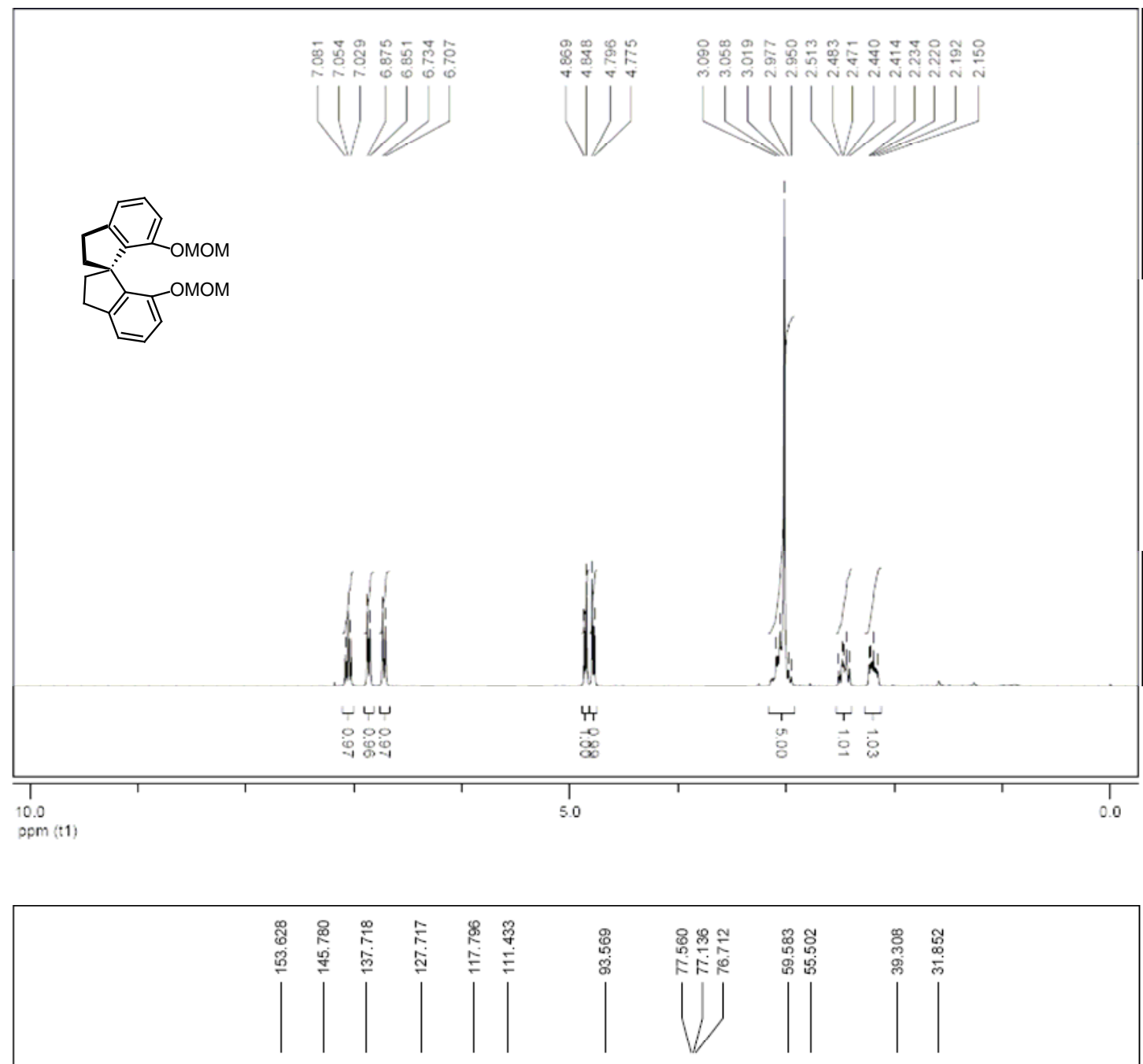

OMOM

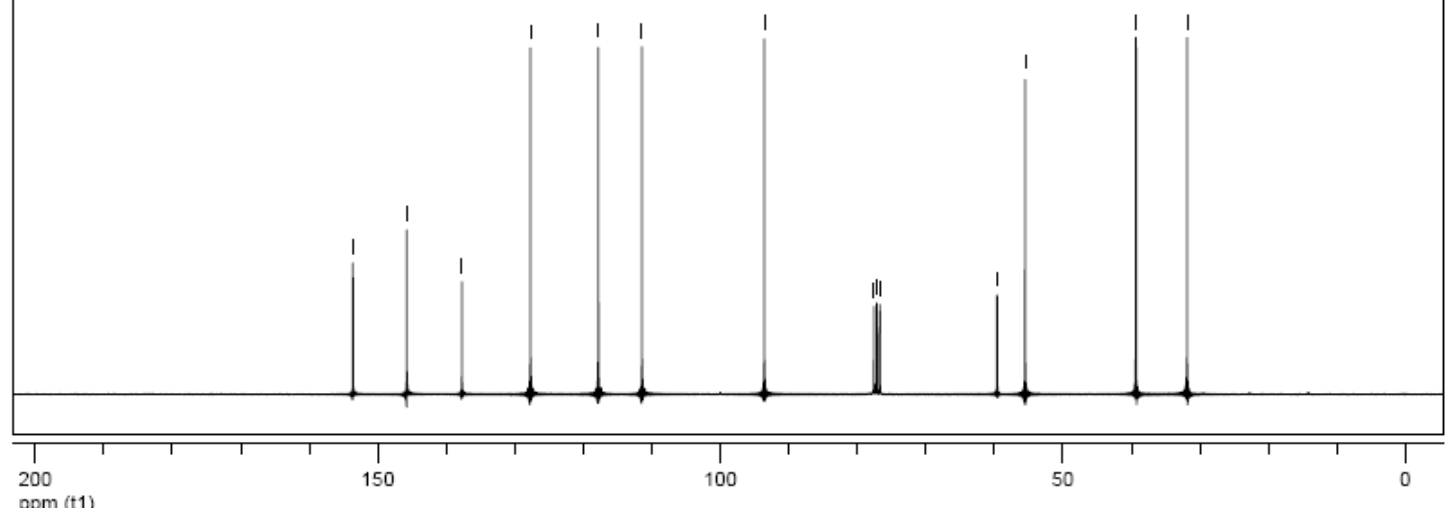



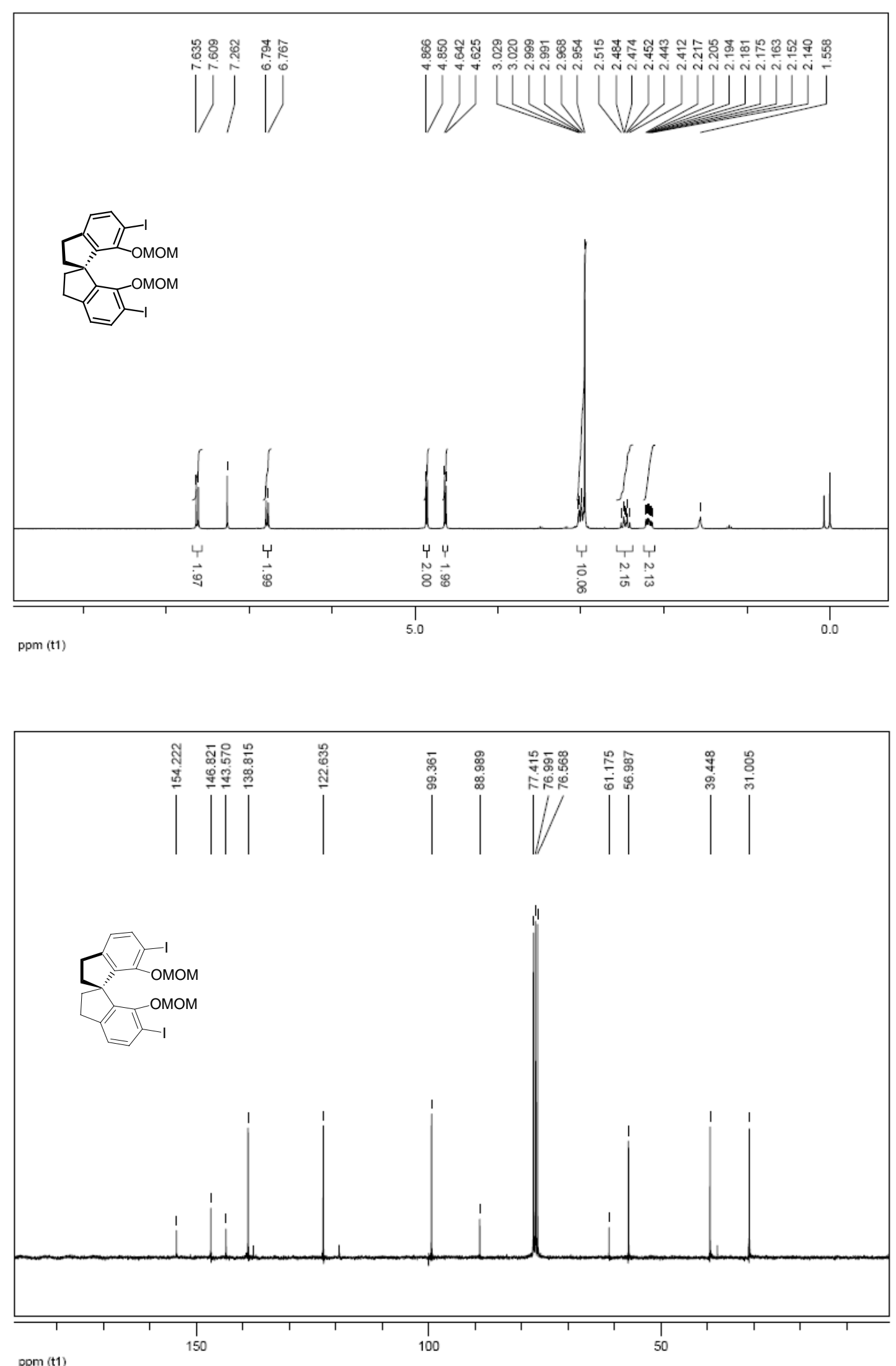

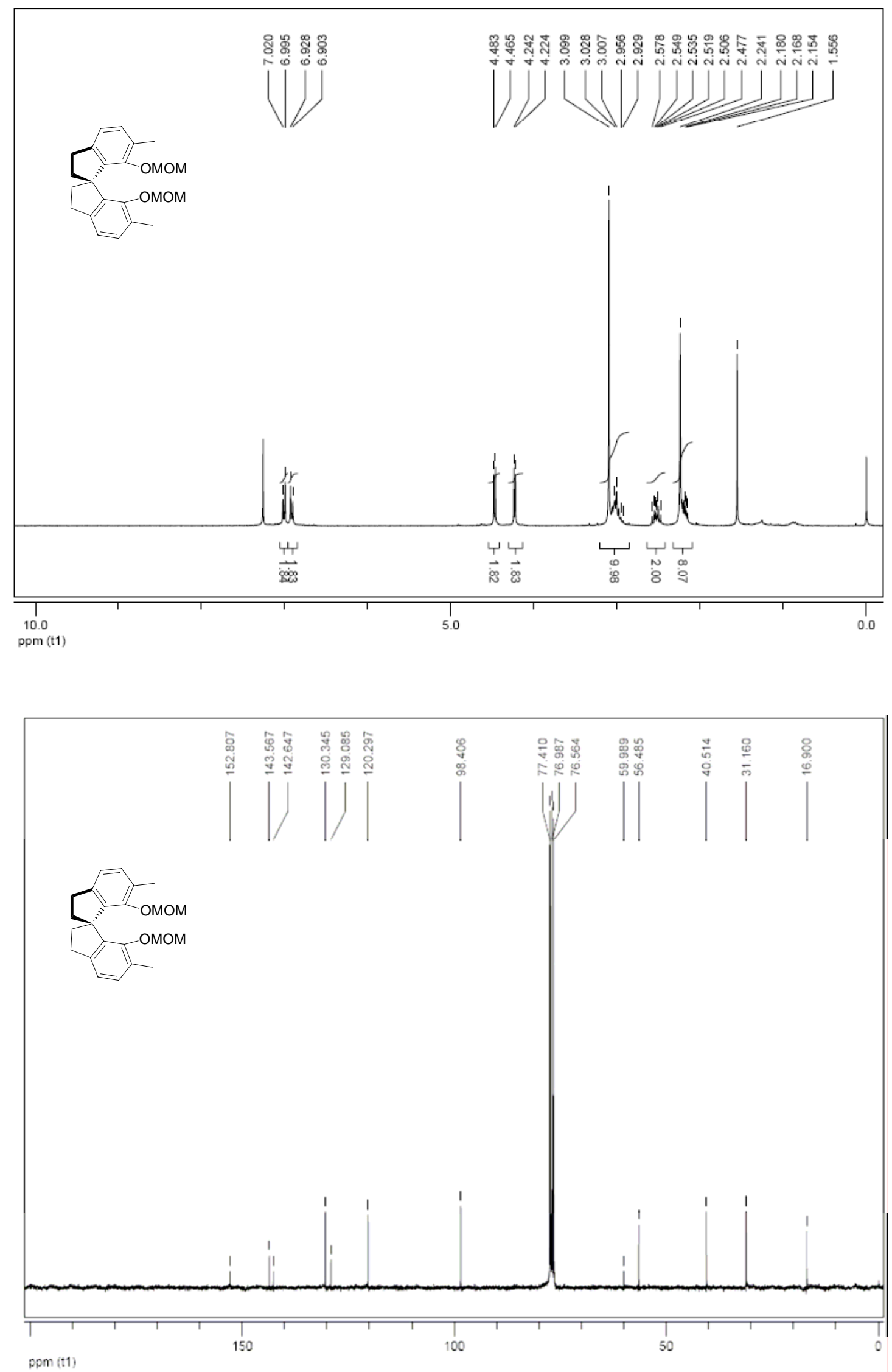

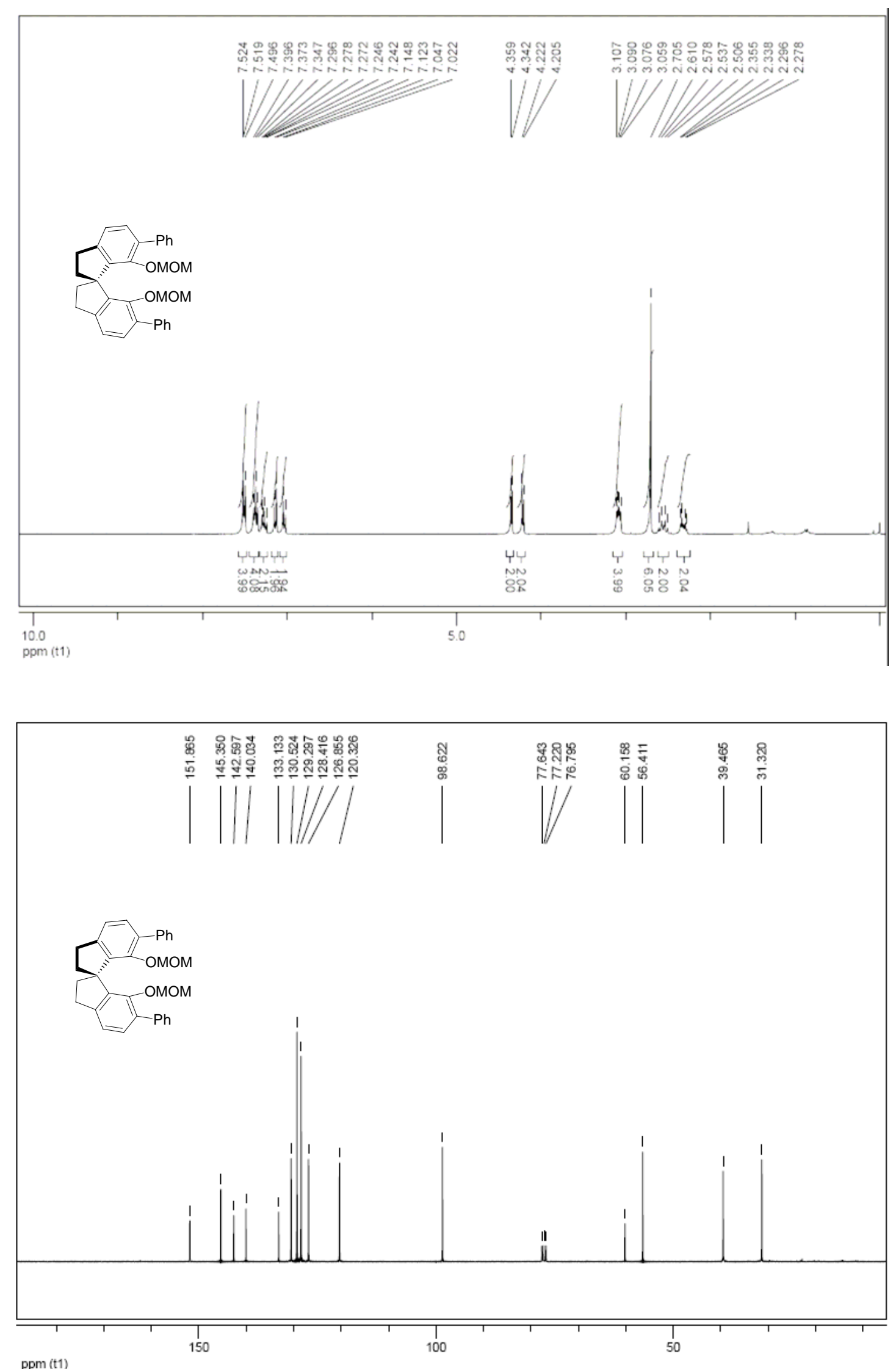

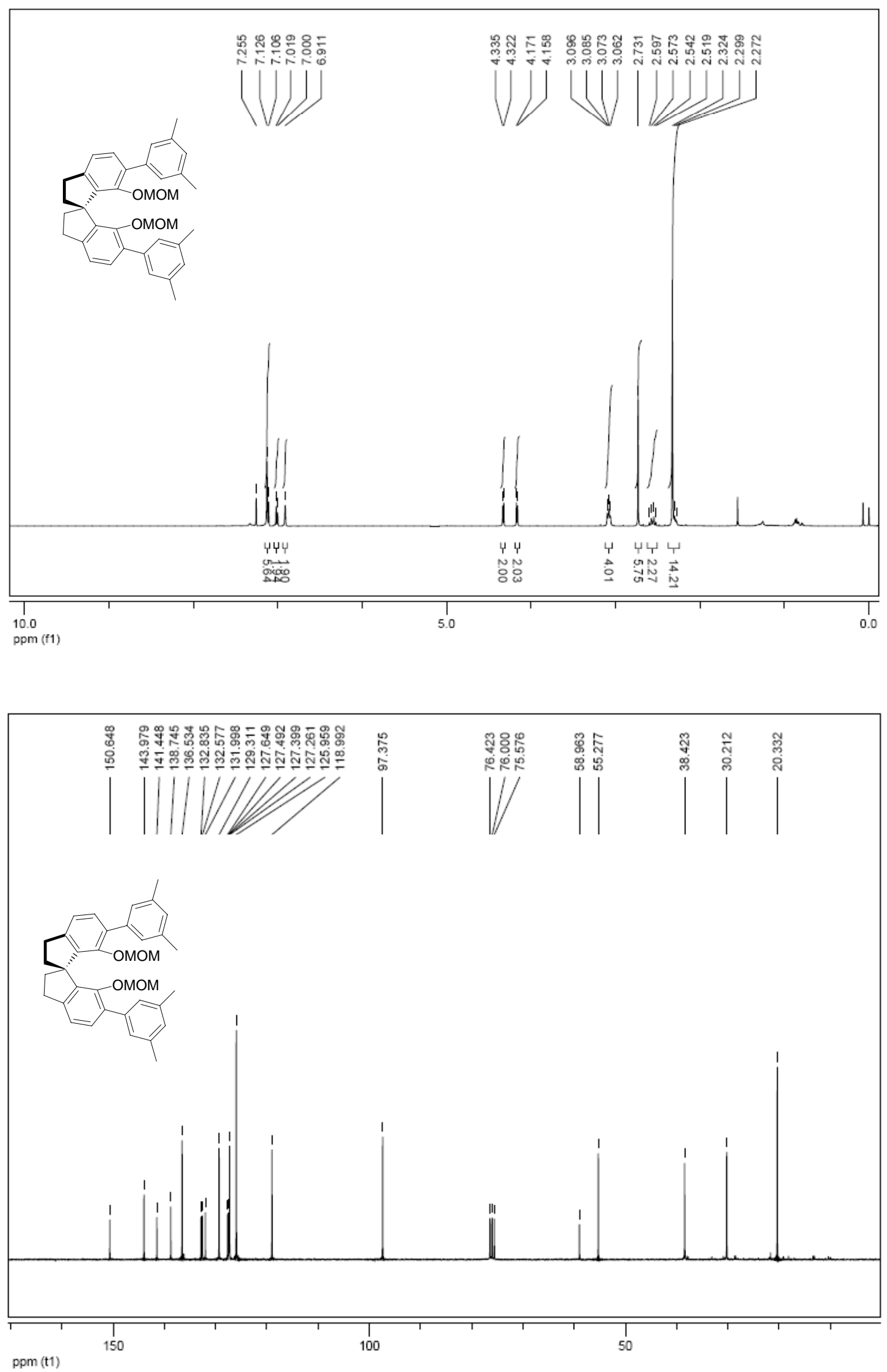

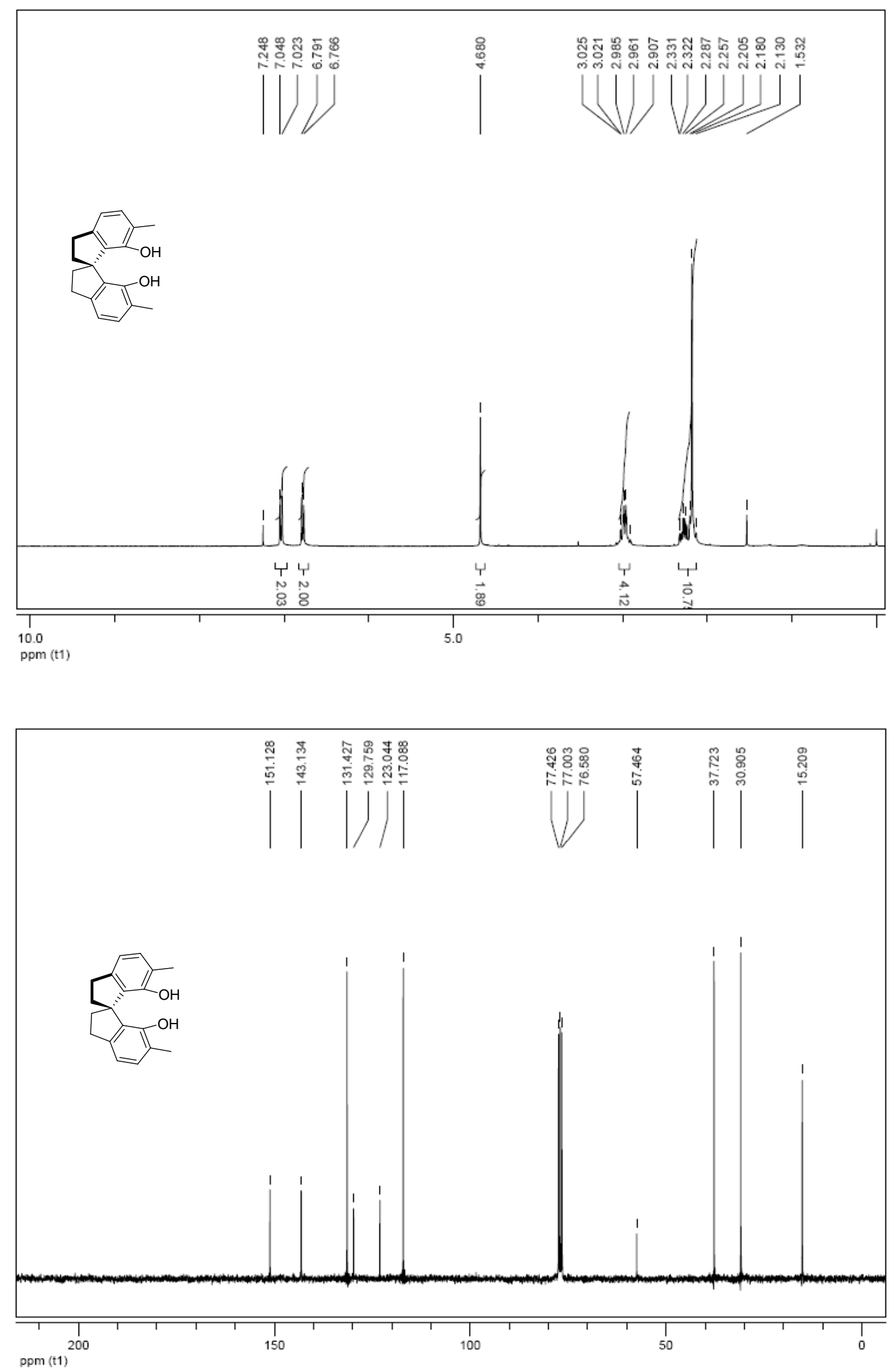

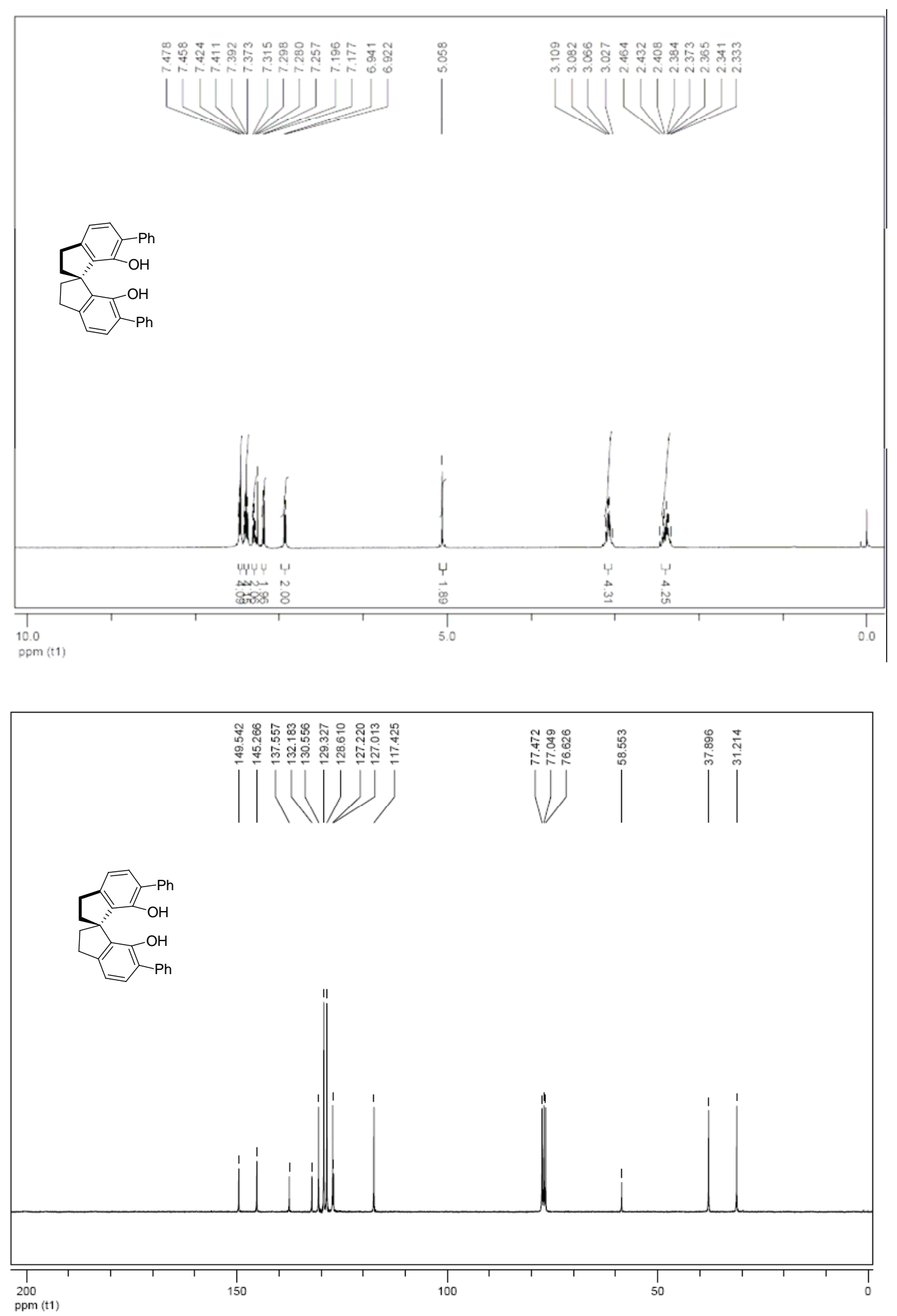

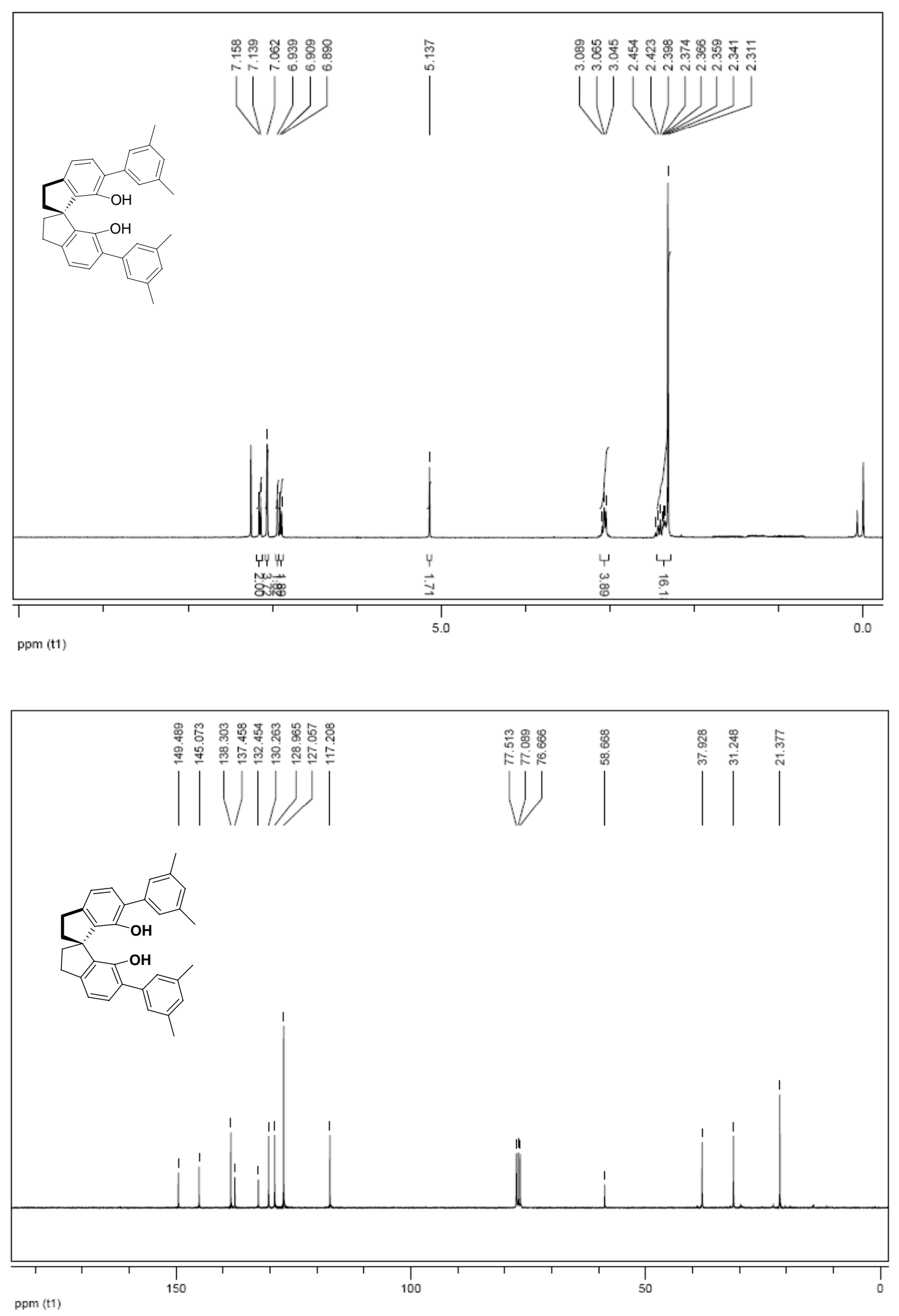

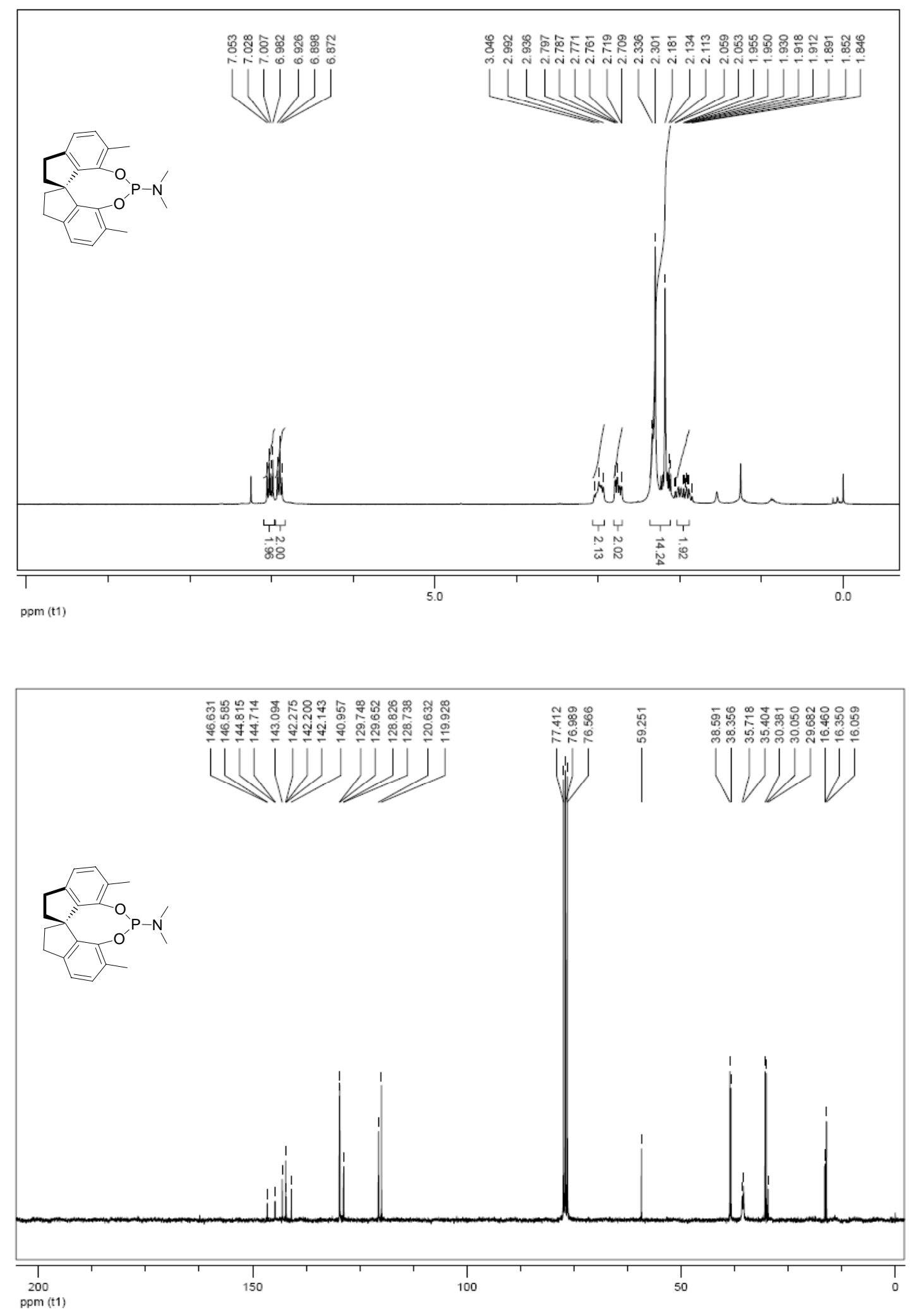


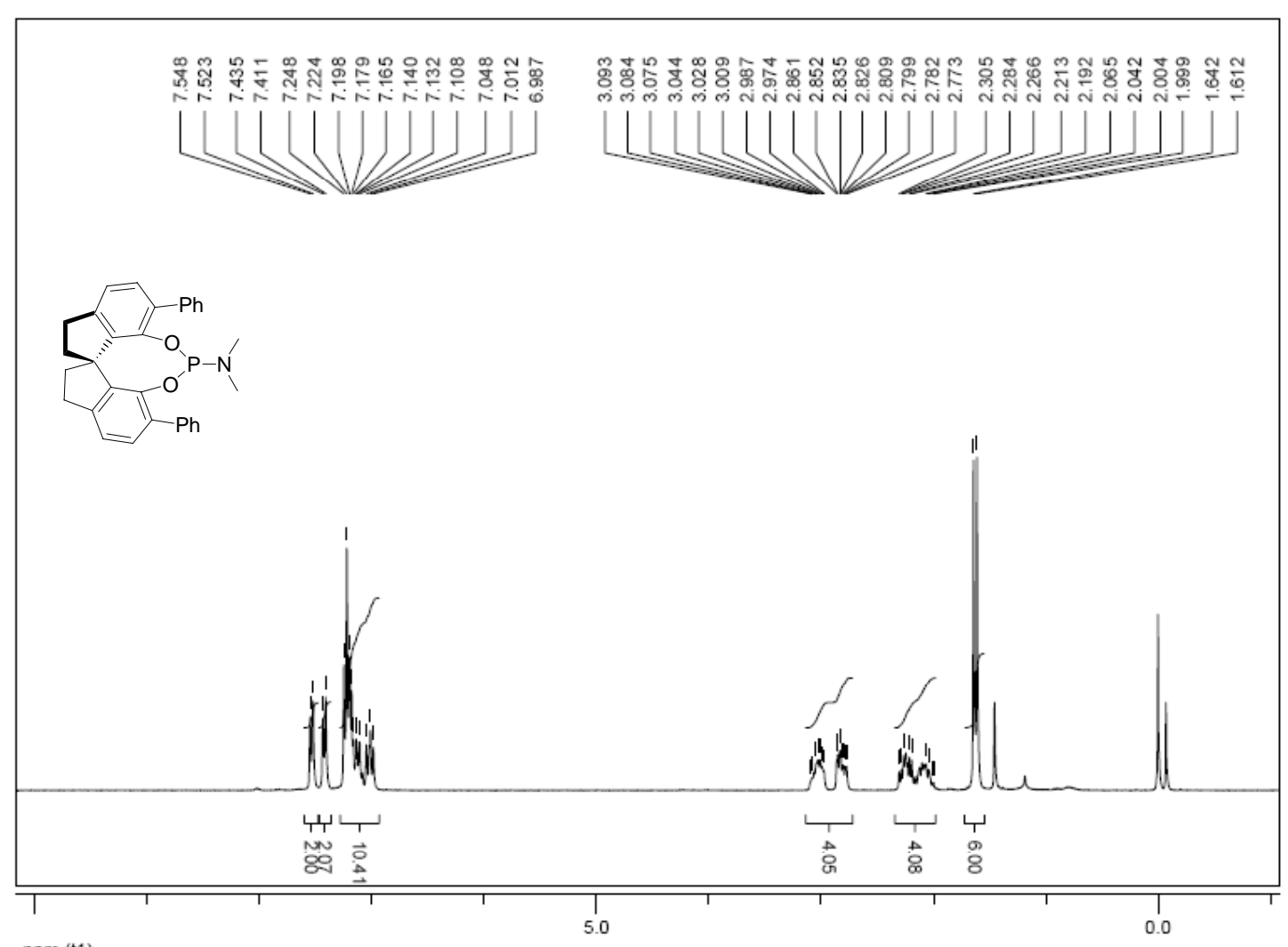

ppm (t1)

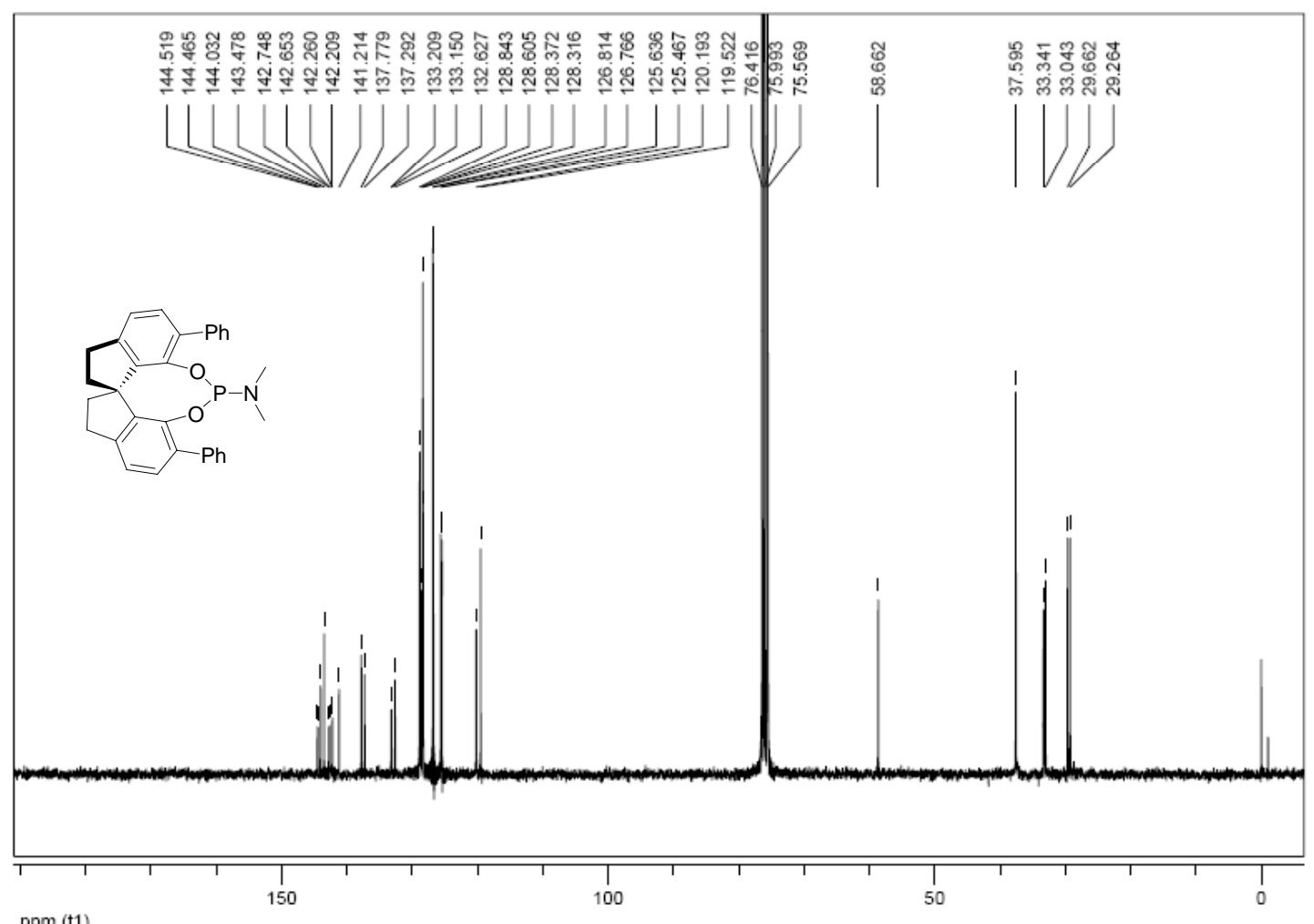



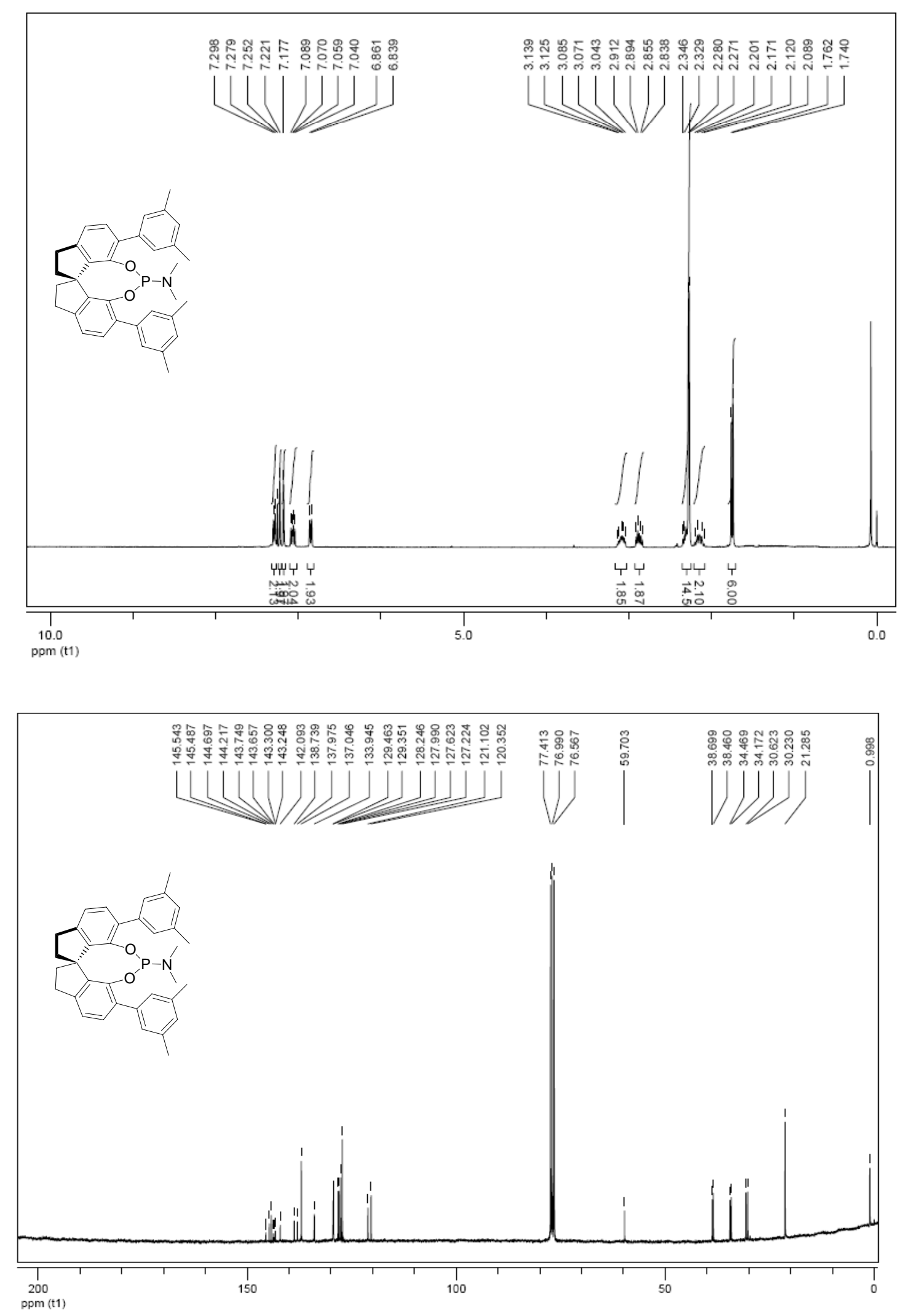

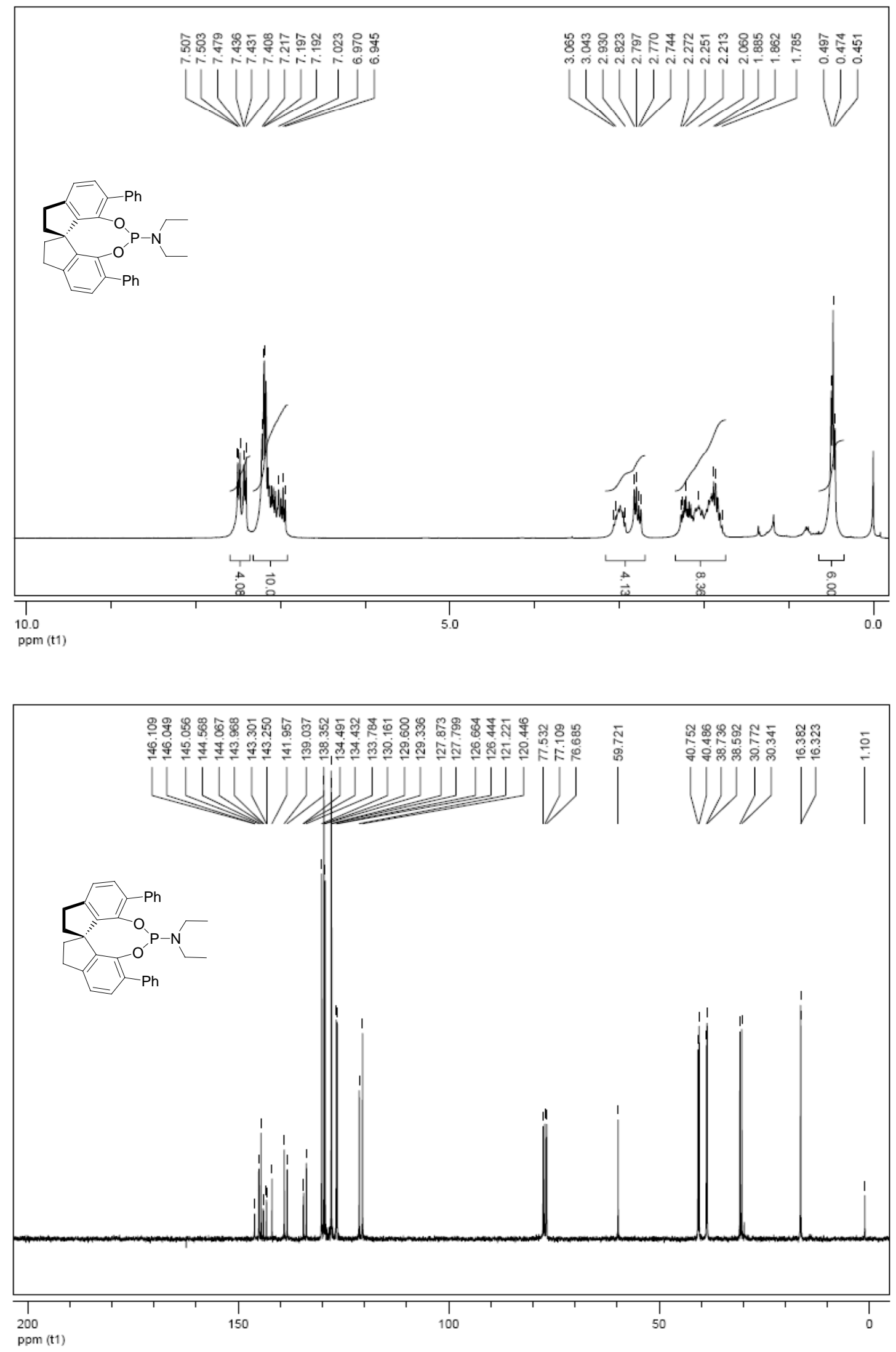

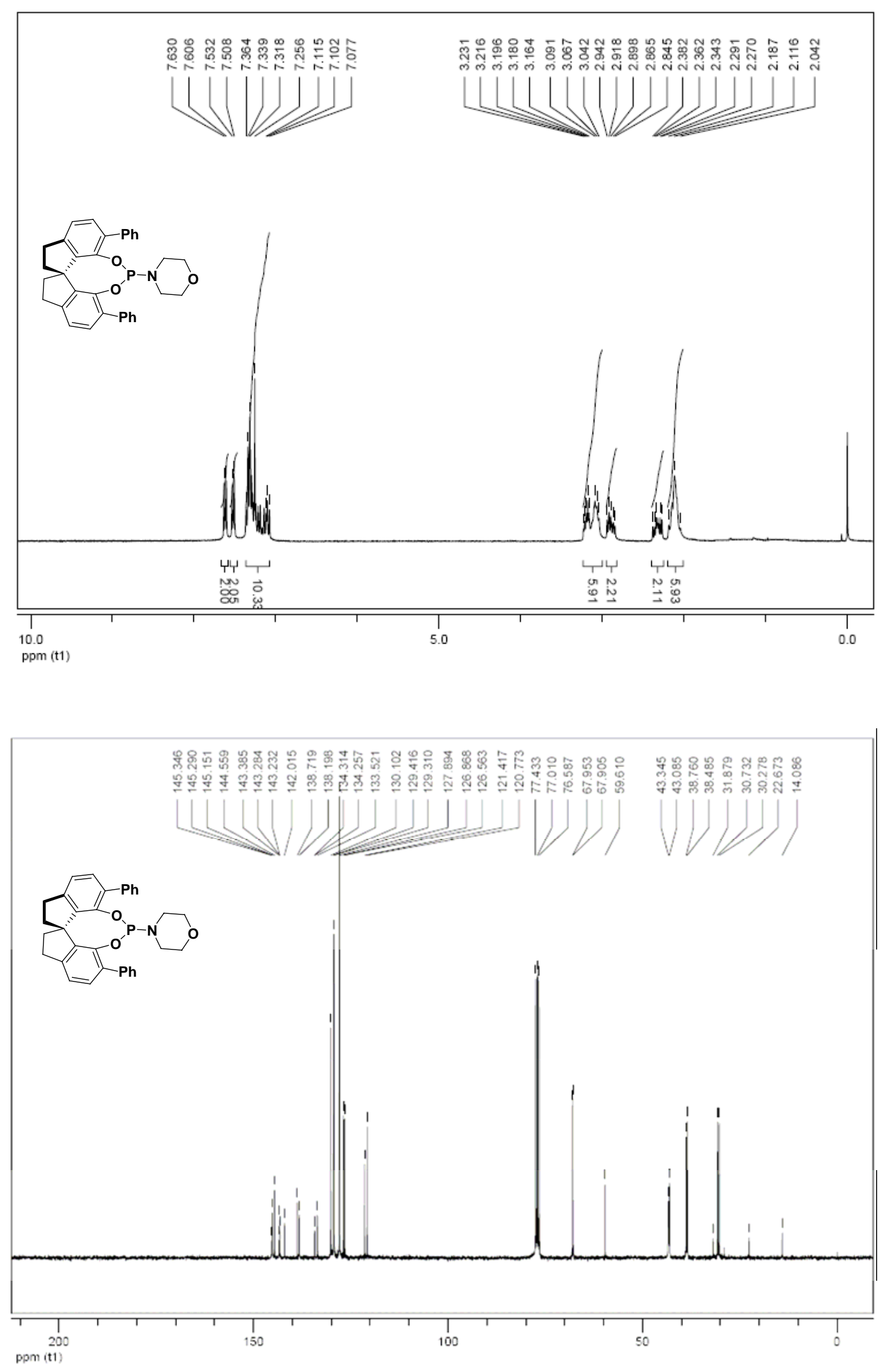
(5) NMR Spectra and HPLC Charts for Chiral Homoallylic Alcohols
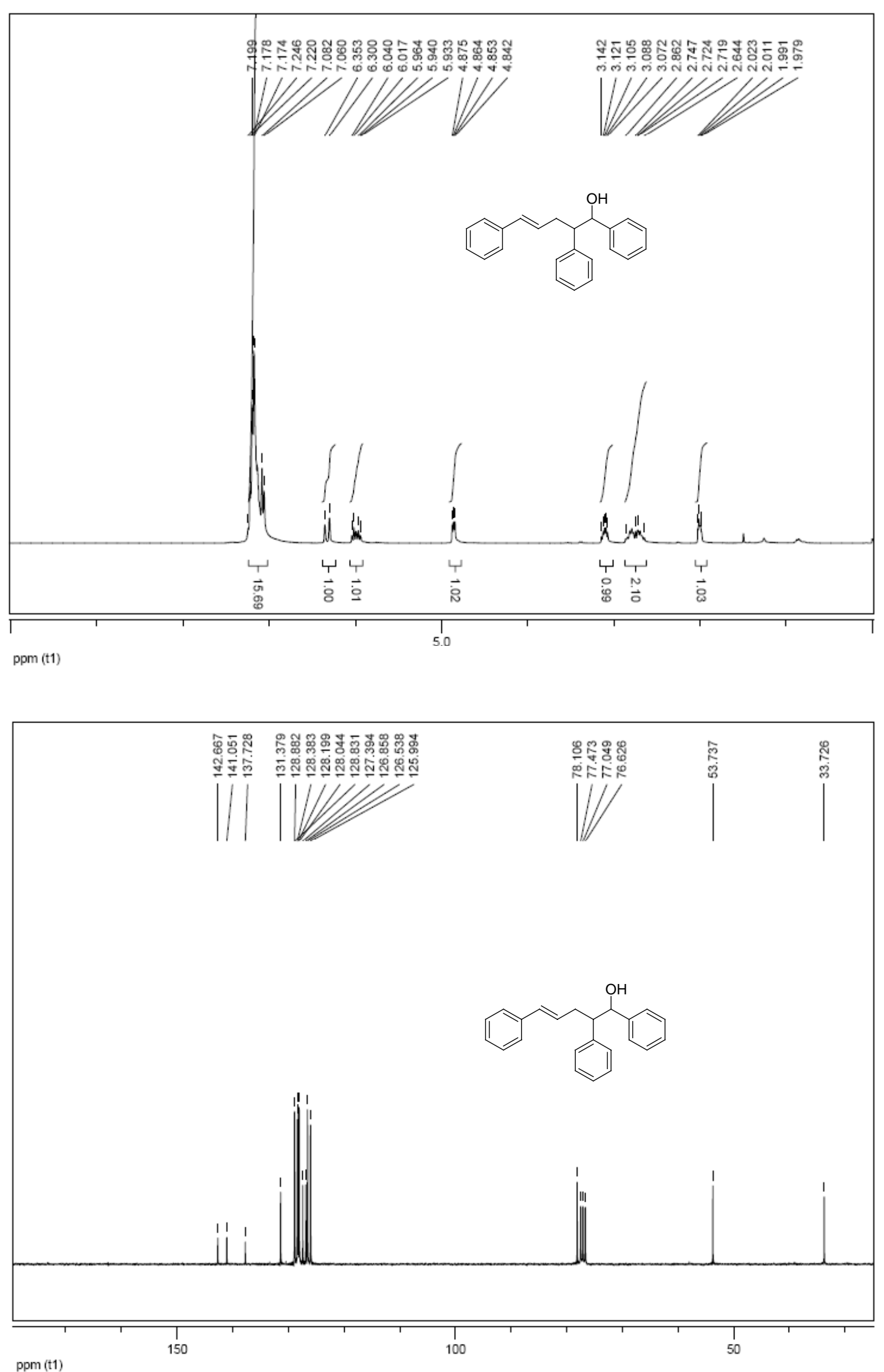

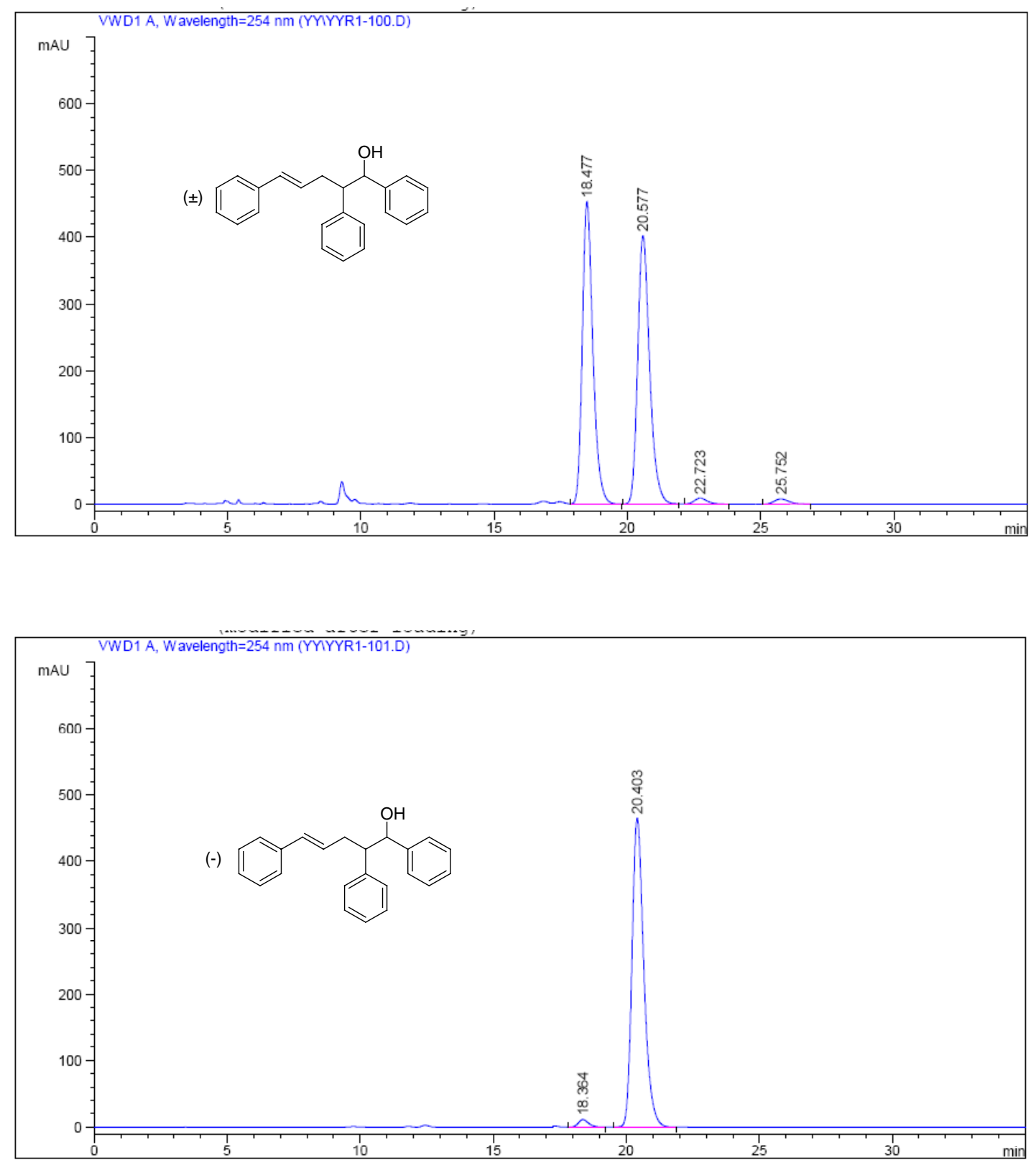

Peak RetTime Type width Area Height Area

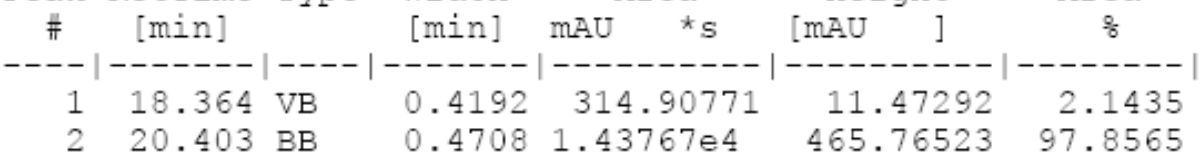
Totals :
$1.46916 e 4 \quad 477.23815$ 

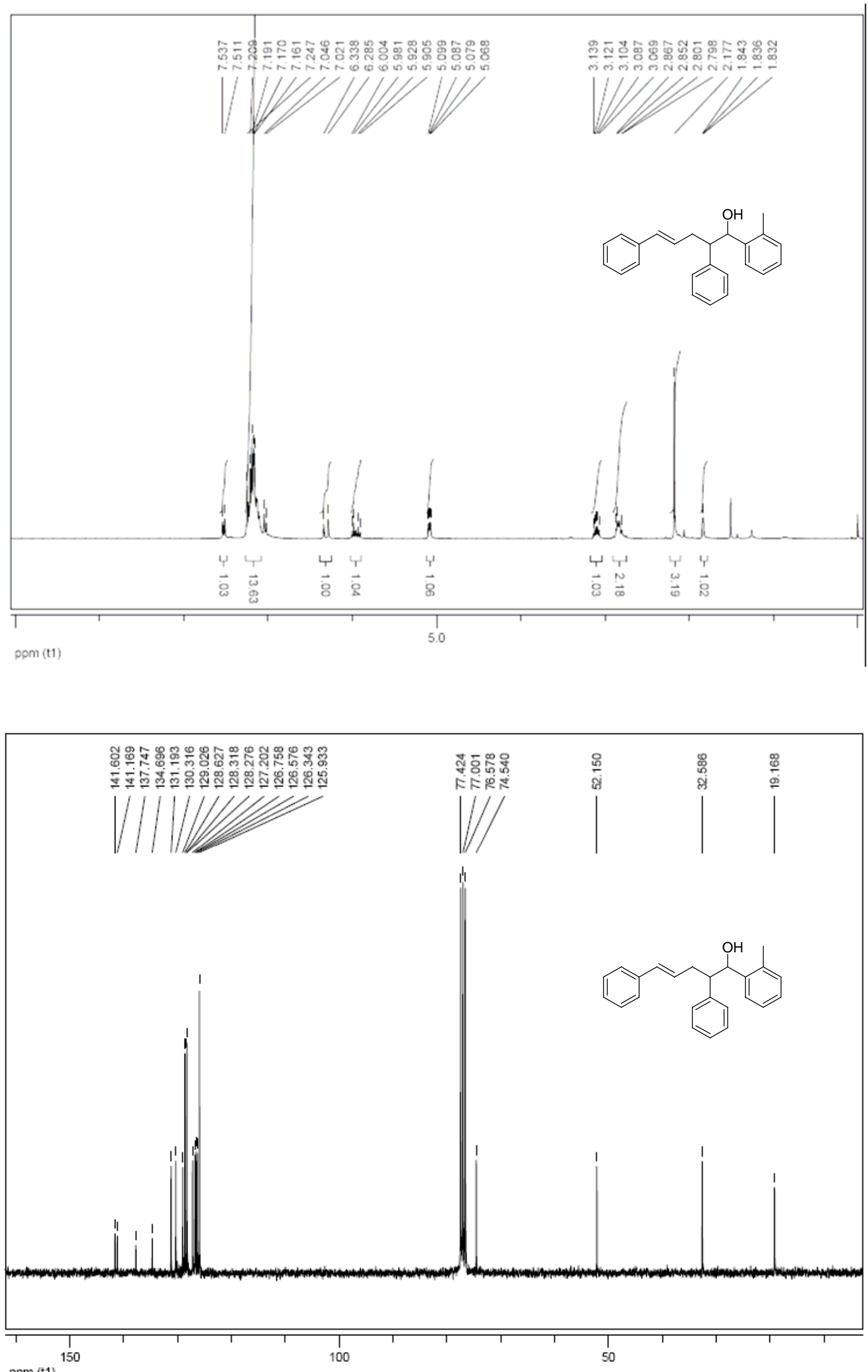

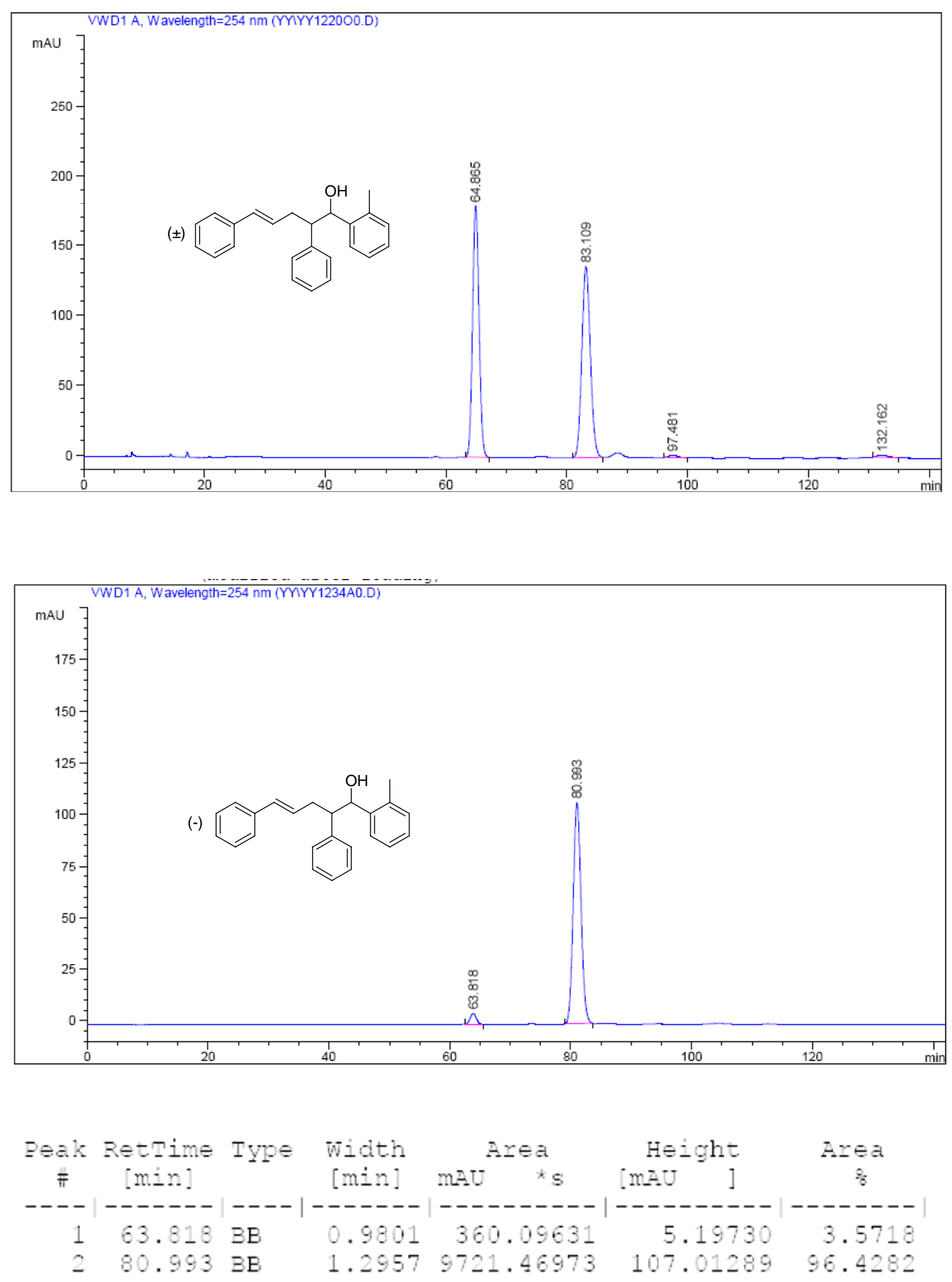

Totals : $\quad 1.00816 e 4 \quad 112.21019$ 

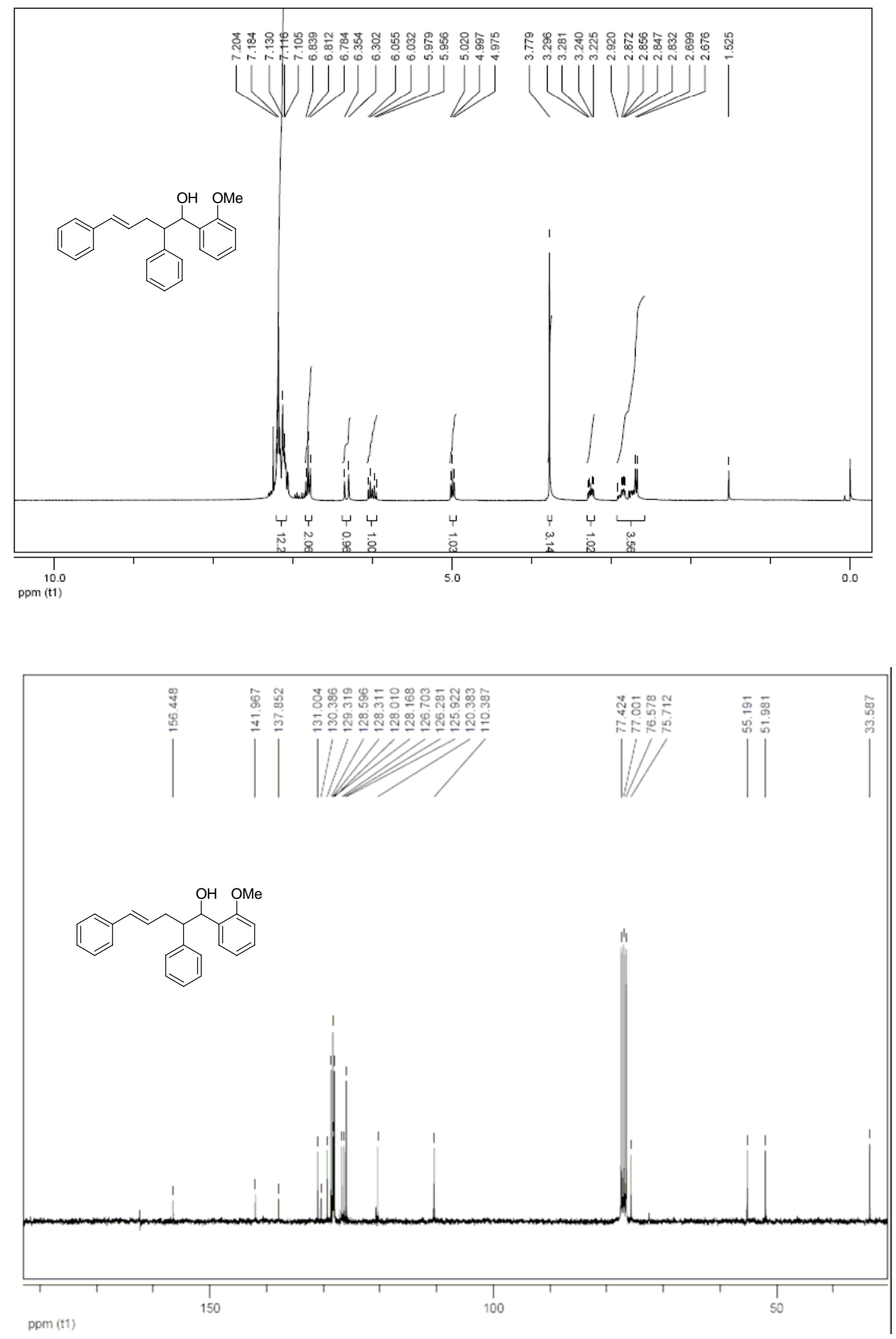

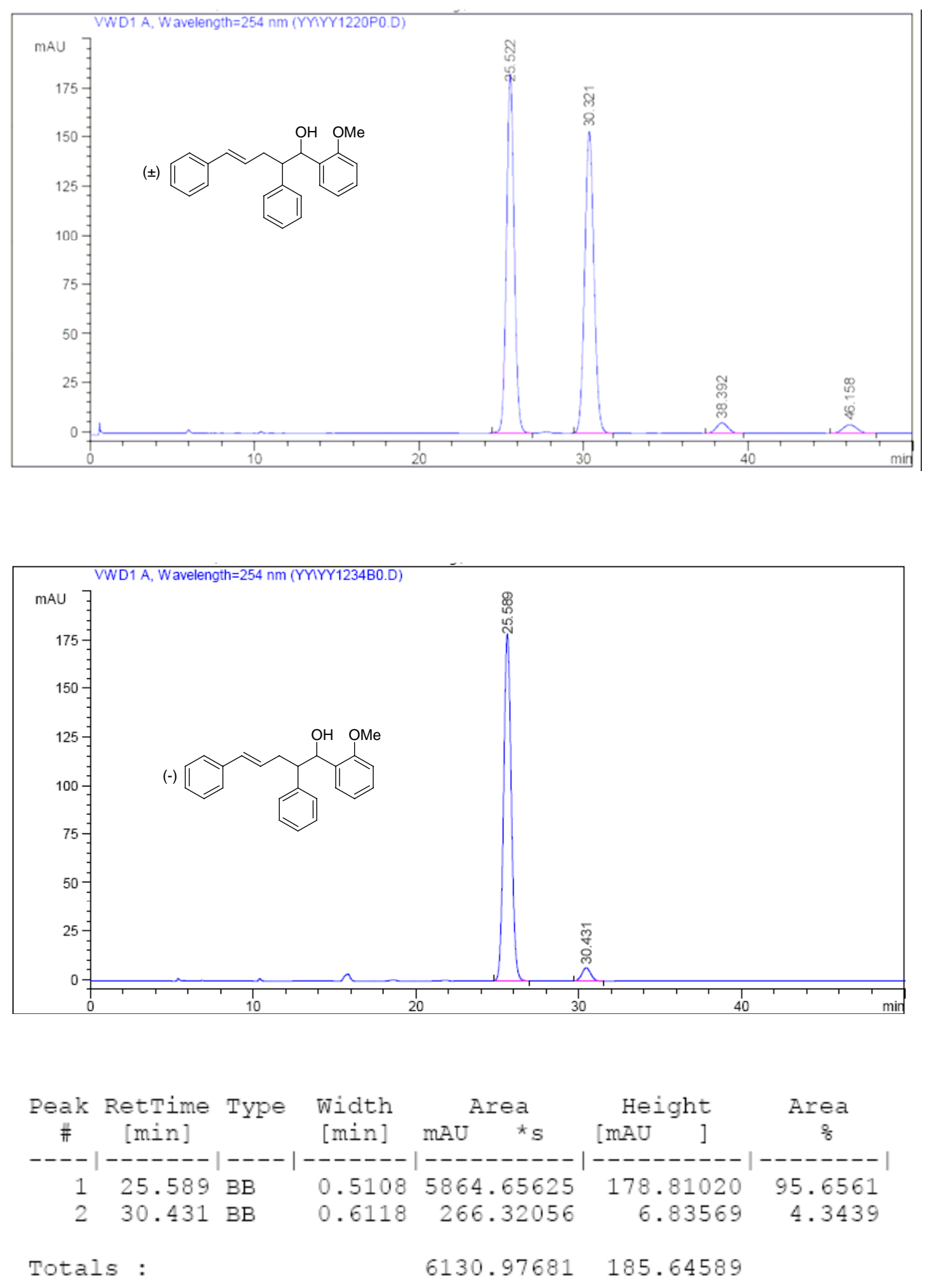


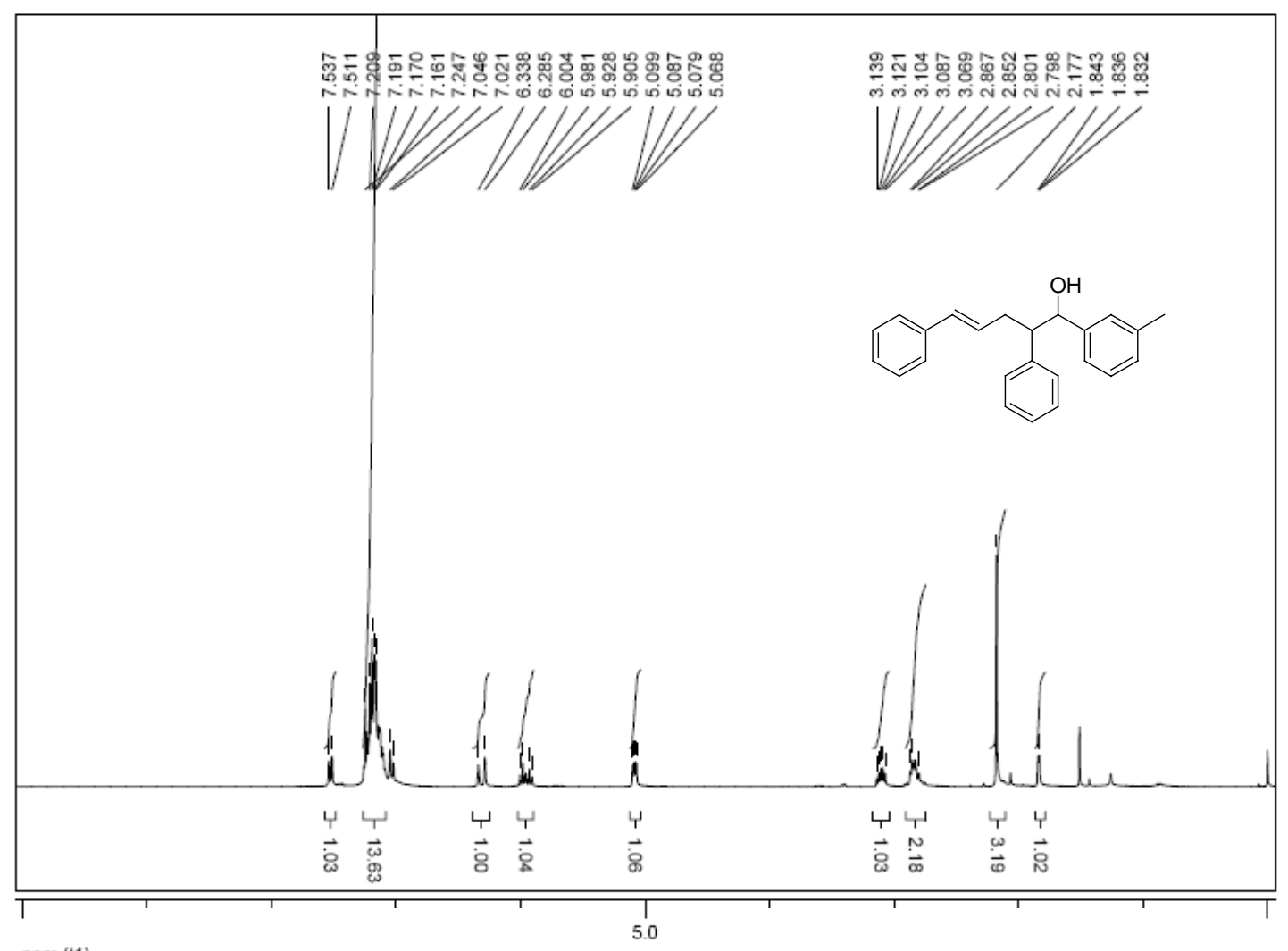

ppm (t1)

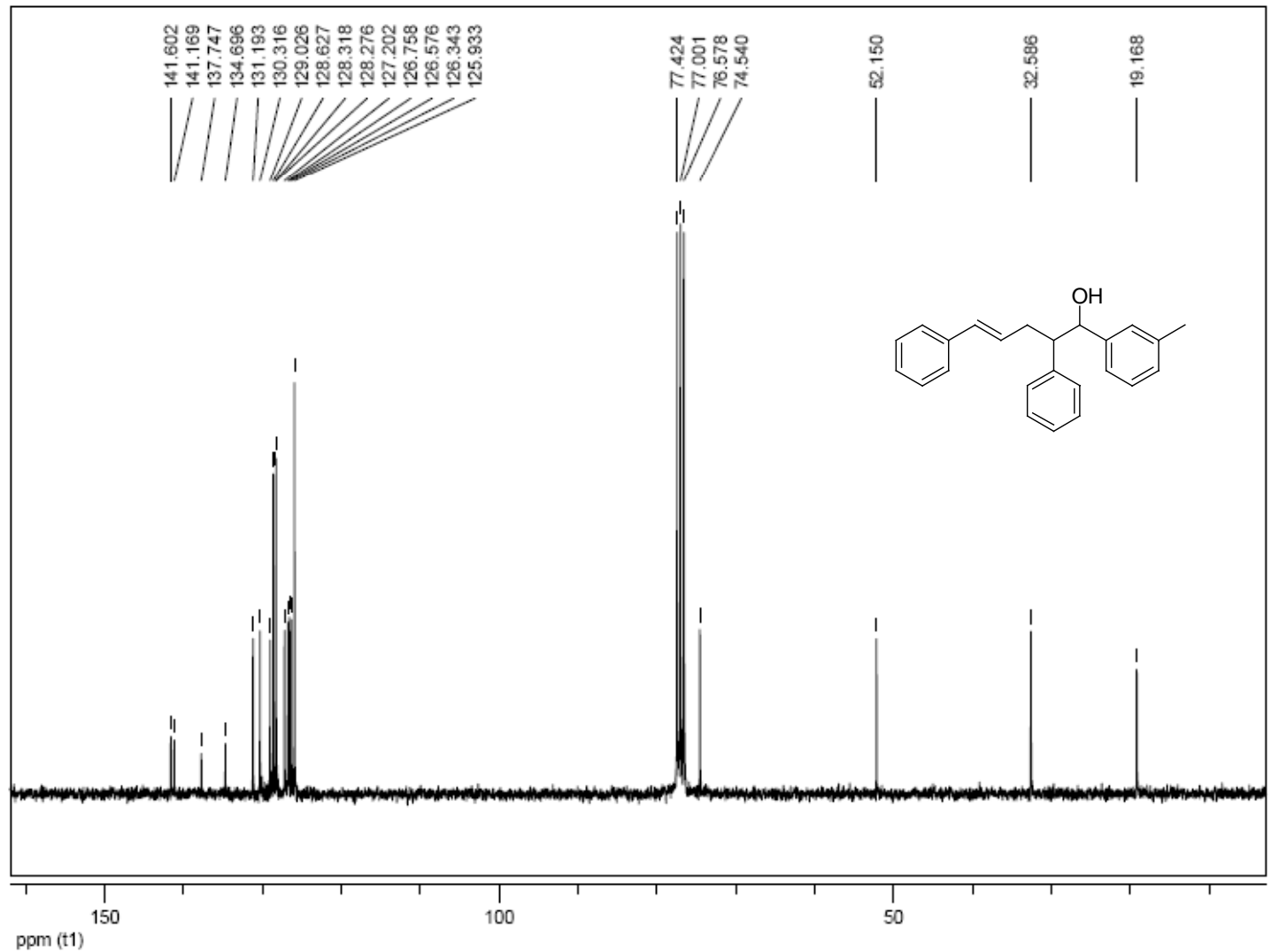



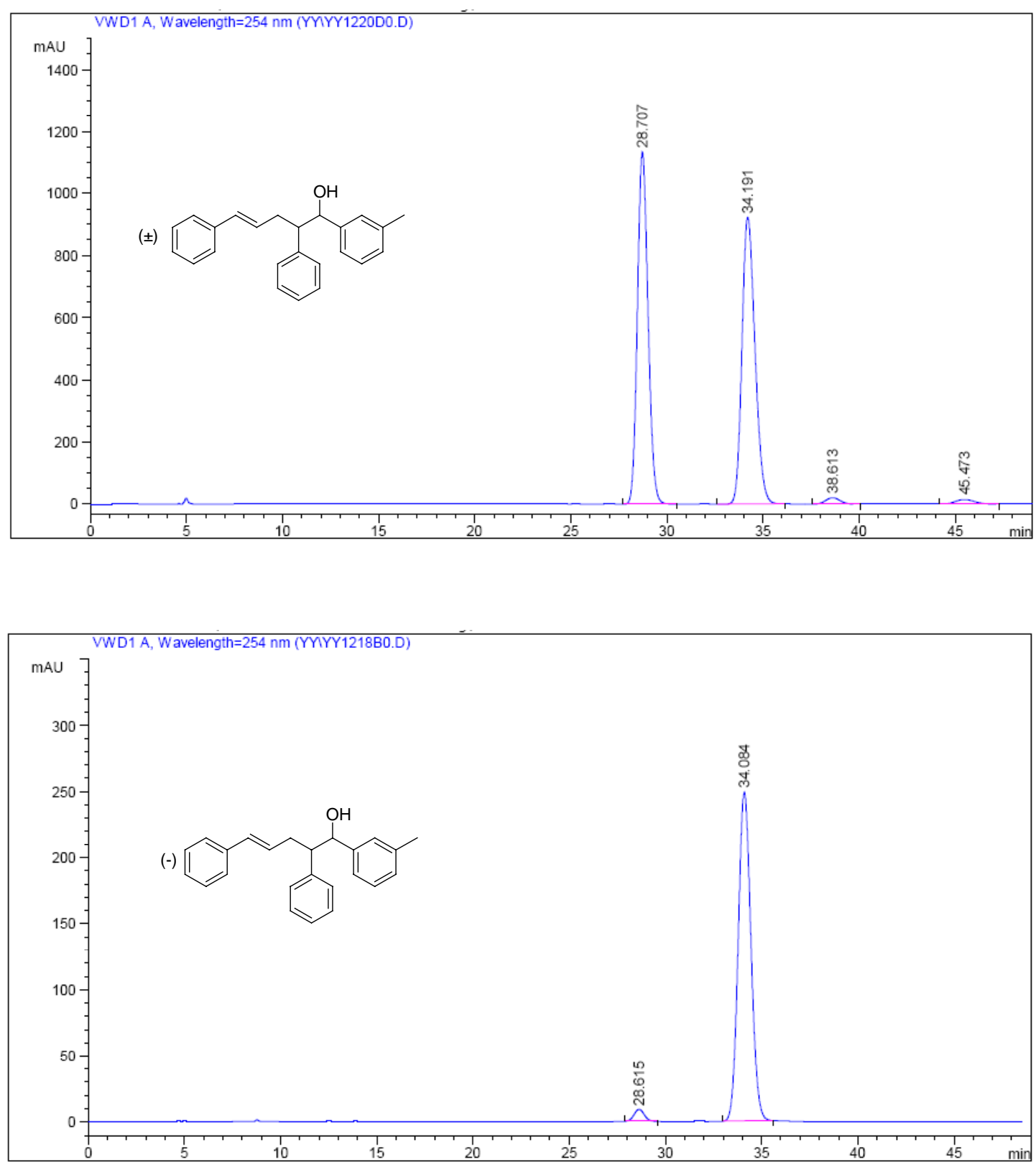

\begin{tabular}{|c|c|c|c|c|c|c|}
\hline$\underset{\#}{\text { Peak }}$ & $\begin{array}{l}\text { RetTime } \\
\text { [min] }\end{array}$ & Type & $\begin{array}{l}\text { Width } \\
{[\mathrm{min}]}\end{array}$ & $\operatorname{mAU}^{\text {Area }}{ }^{\star} \mathrm{s}$ & $\begin{array}{l}\text { Height } \\
{[\mathrm{mAU}]}\end{array}$ & $\begin{array}{c}\text { Area } \\
\frac{8}{8}\end{array}$ \\
\hline $\begin{array}{l}1 \\
2\end{array}$ & $\begin{array}{l}28.615 \\
34.084\end{array}$ & $\begin{array}{l}\mathrm{BB} \\
\mathrm{BB}\end{array}$ & $\begin{array}{l}0.5799 \\
0.7104\end{array}$ & $\begin{array}{r}335.43201 \\
1.13922 e 4\end{array}$ & $\begin{array}{r}8.98025 \\
248.77248\end{array}$ & $\begin{array}{r}2.8602 \\
97.1398\end{array}$ \\
\hline tal & : & & & $1.17277 e 4$ & 257.75273 & \\
\hline
\end{tabular}



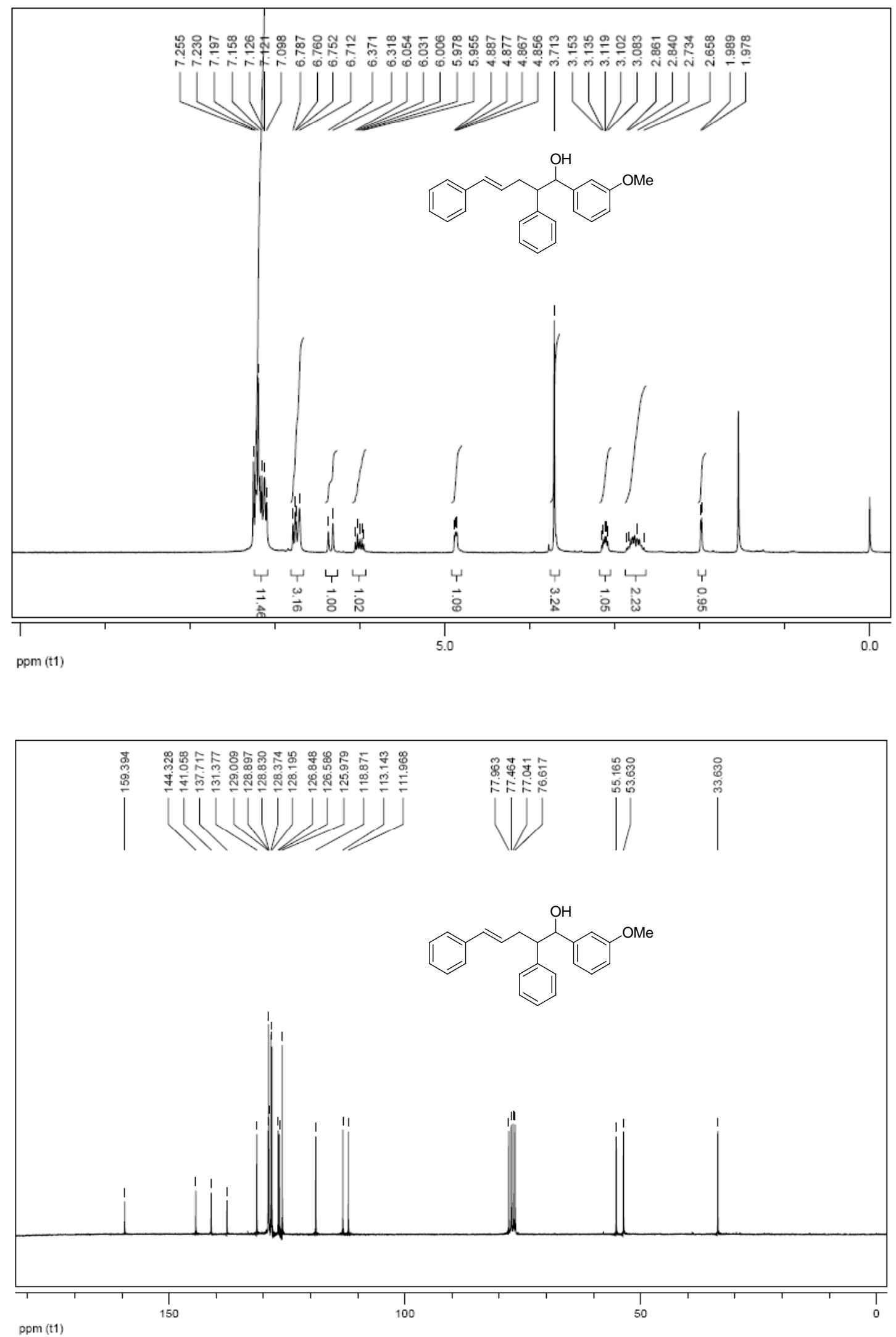

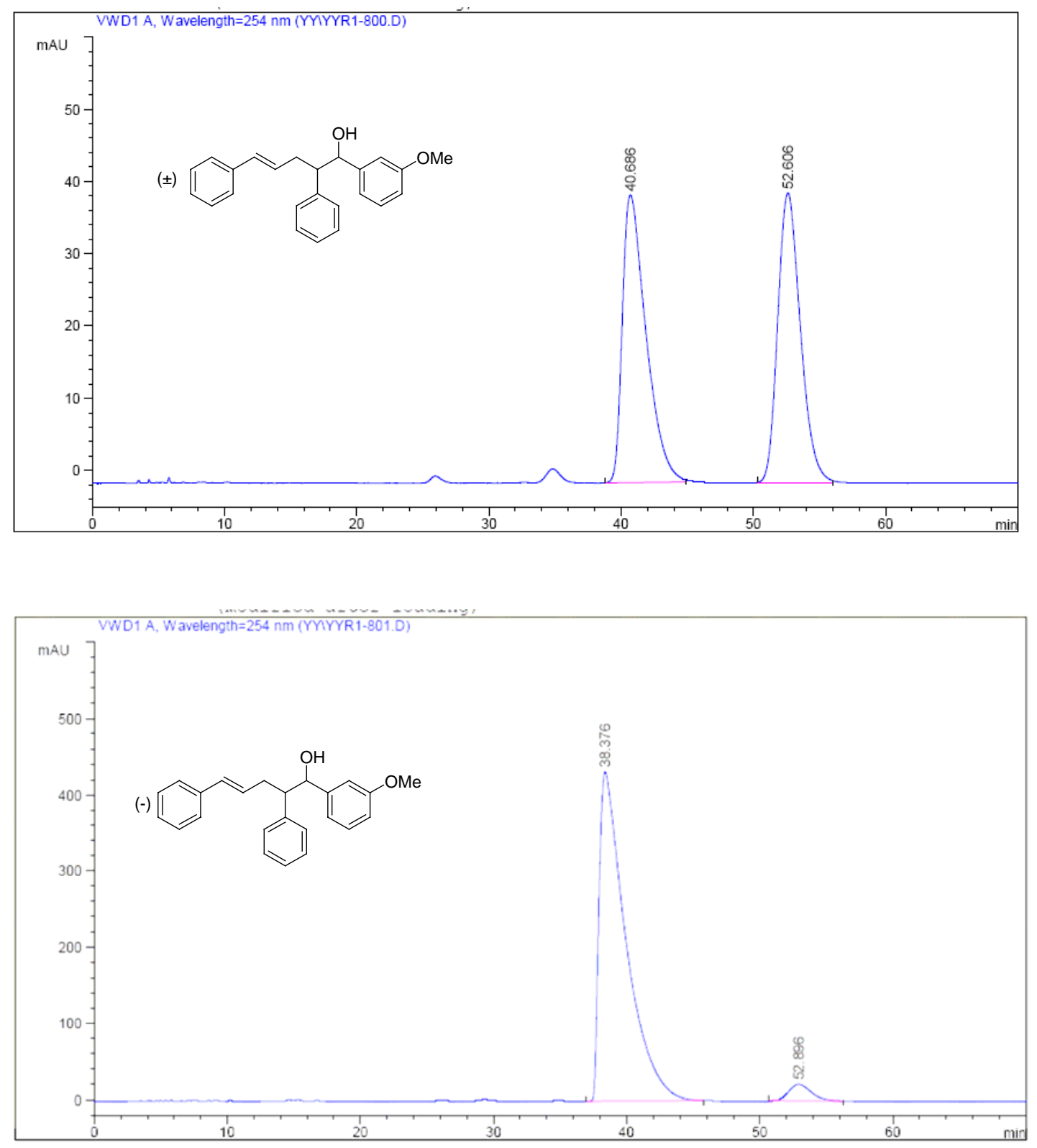

\begin{tabular}{|c|c|c|c|c|c|c|}
\hline $\begin{array}{c}\text { Peak } \\
\quad \#\end{array}$ & $\begin{array}{c}\text { Retrime } \\
\text { [min] }\end{array}$ & Type & $\begin{array}{l}\text { Width } \\
\text { [min] }\end{array}$ & $\mathrm{mAU}^{\text {Area }}{ }^{*} \mathrm{~s}$ & $\underset{\mathrm{mAU}}{\mathrm{Height}}$ & $\begin{array}{c}\text { Area } \\
\frac{\%}{6}\end{array}$ \\
\hline- & $\begin{array}{l}38.376 \\
52.896\end{array}$ & $\begin{array}{l}P B \\
B B\end{array}$ & $\begin{array}{l}2.9179 \\
1.8390\end{array}$ & $\begin{array}{l}6.05543 e 4 \\
2606.36865\end{array}$ & $\begin{array}{r}432.47498 \\
21.77598\end{array}$ & $\begin{array}{r}95.8734 \\
4.1266\end{array}$ \\
\hline 0 & & & & $6.31607 e 4$ & 454.25095 & \\
\hline
\end{tabular}



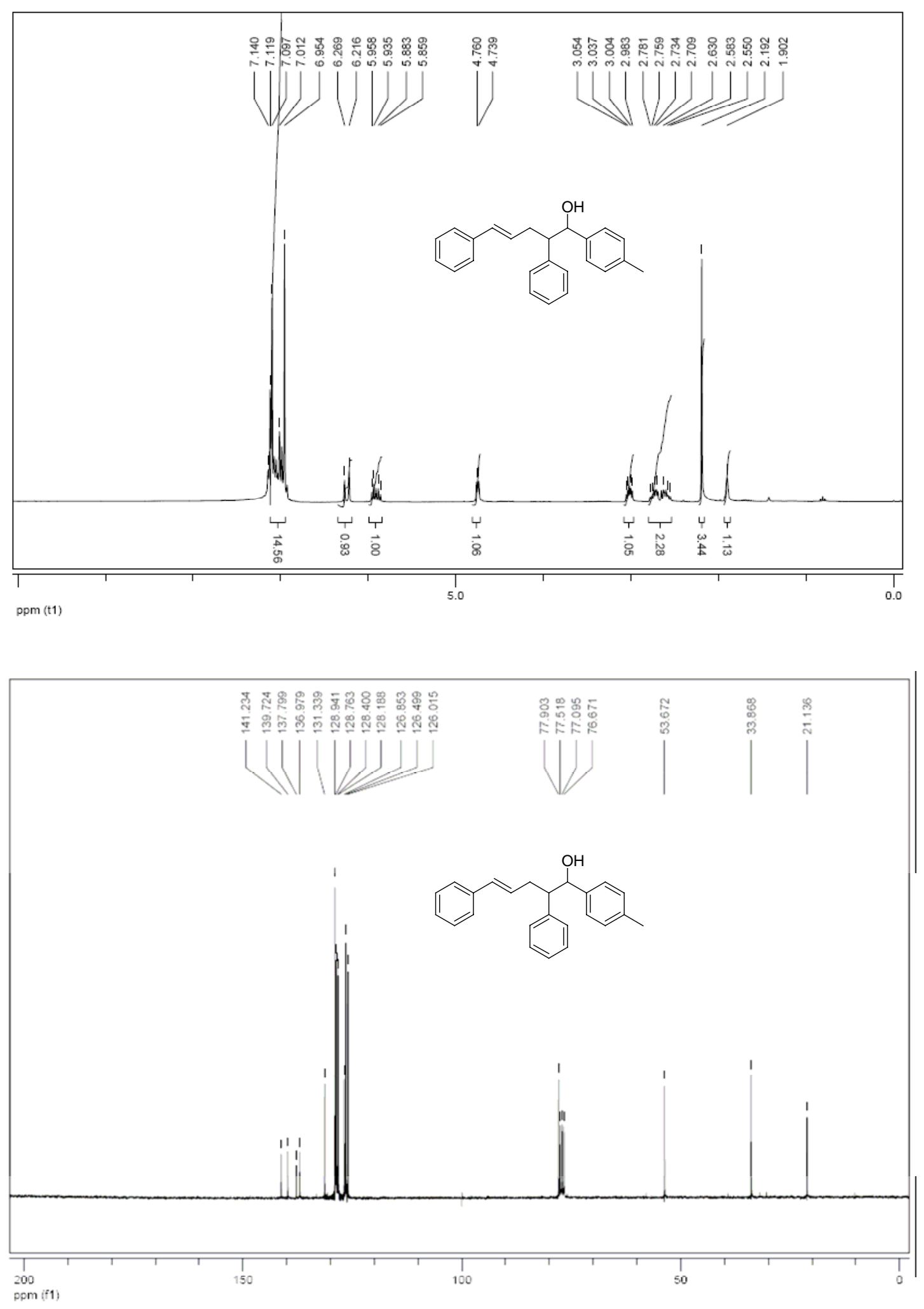

- S39 - 

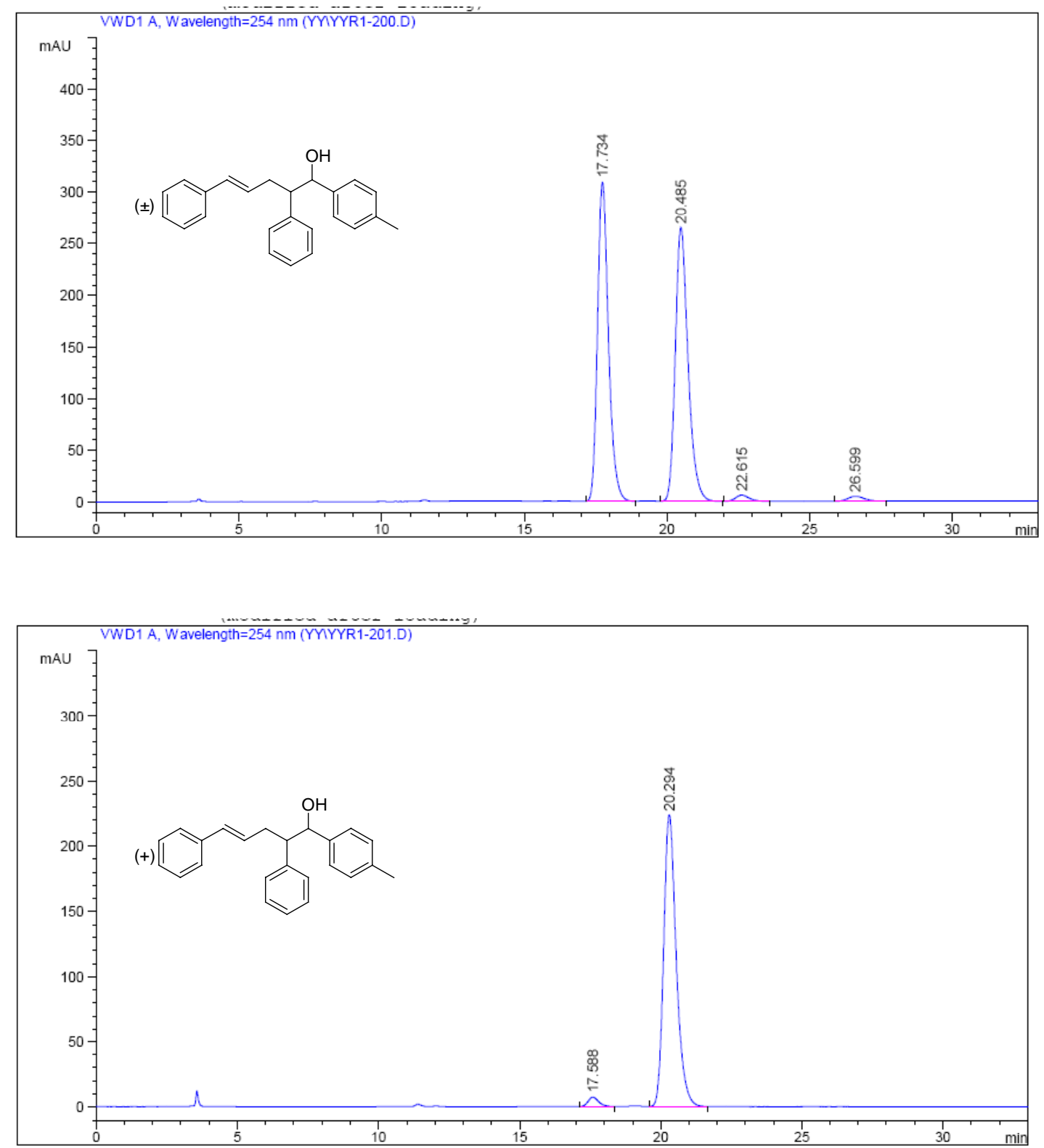

Signal 1: VWD1 A, Wavelength=254 nm

\begin{tabular}{|c|c|c|c|c|c|c|}
\hline Peak & RetTime & Type & width & Area & Height & Area \\
\hline$\#$ & [min] & & {$[\min ]$} & $\mathrm{mAU}$ & {$[\mathrm{mAU}$} & $\frac{8}{8}$ \\
\hline 2 & $\begin{array}{l}17.588 \\
20.294\end{array}$ & $\begin{array}{l}\mathrm{BB} \\
\mathrm{BB}\end{array}$ & $\begin{array}{l}0.3978 \\
0.4718\end{array}$ & $\begin{array}{r}187.31834 \\
6929.49561\end{array}$ & $\begin{array}{r}7.17324 \\
223.87173\end{array}$ & $\begin{array}{r}2.6321 \\
97.3679\end{array}$ \\
\hline Tot & : & & & 7116.81395 & 231.04497 & \\
\hline
\end{tabular}



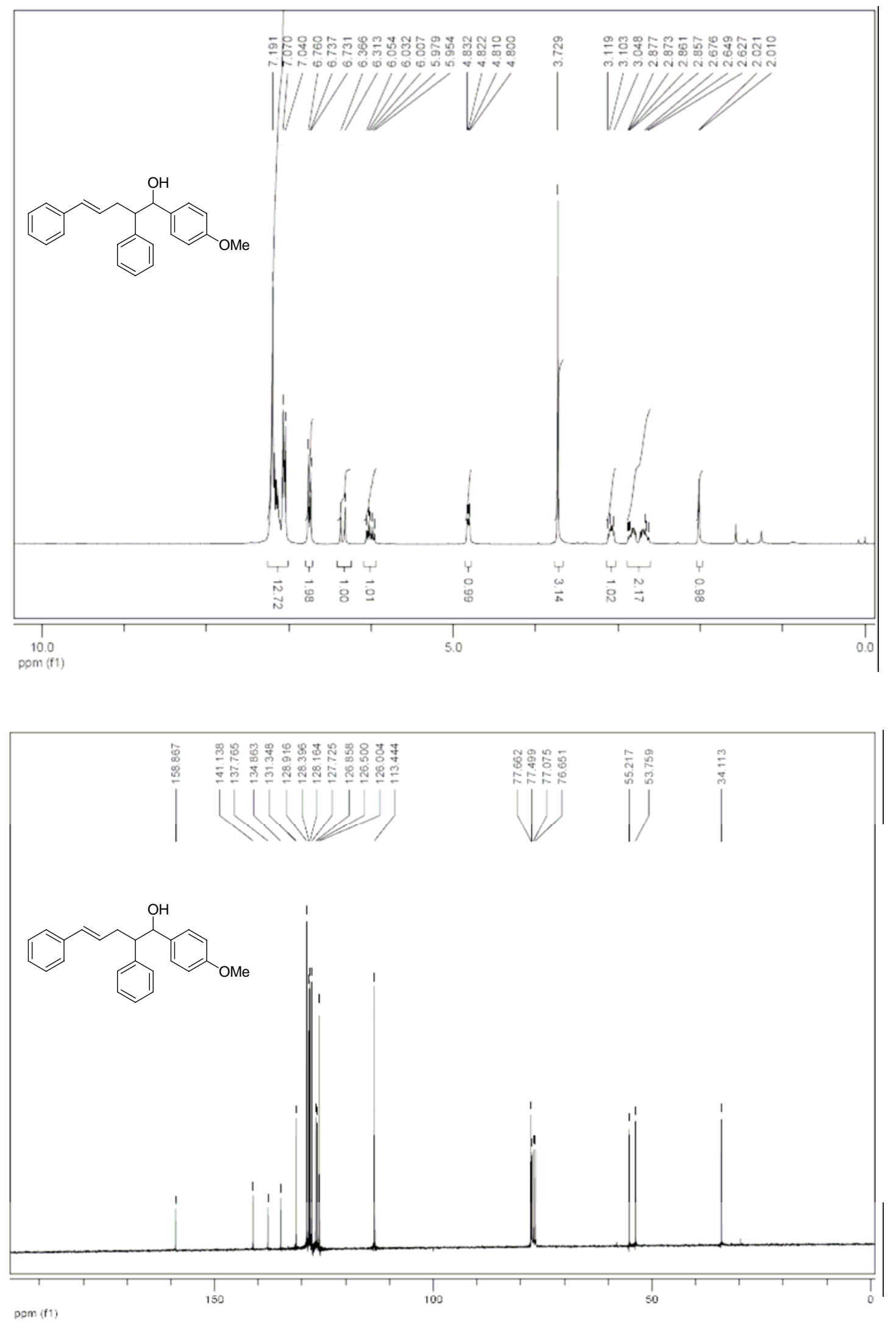

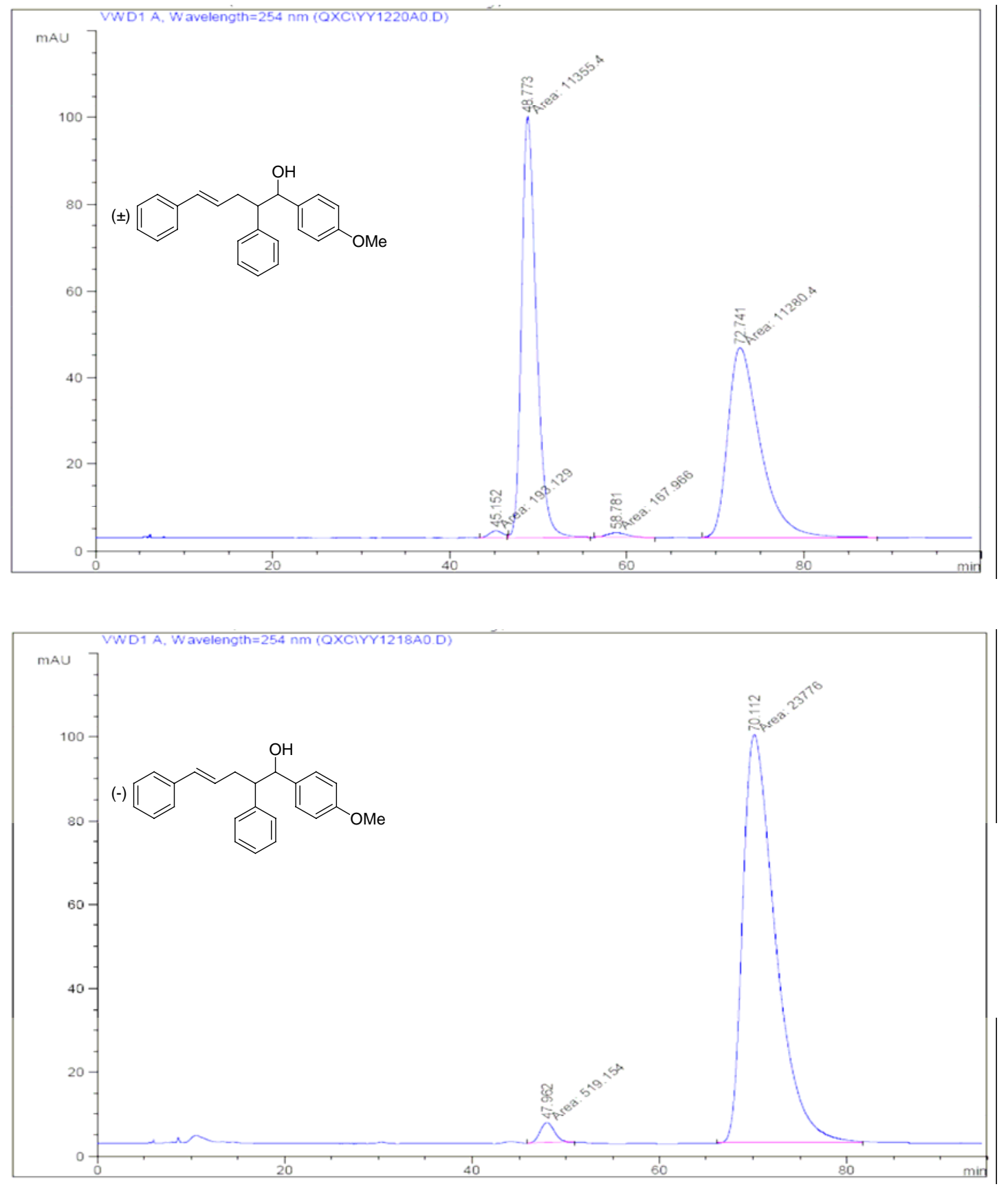

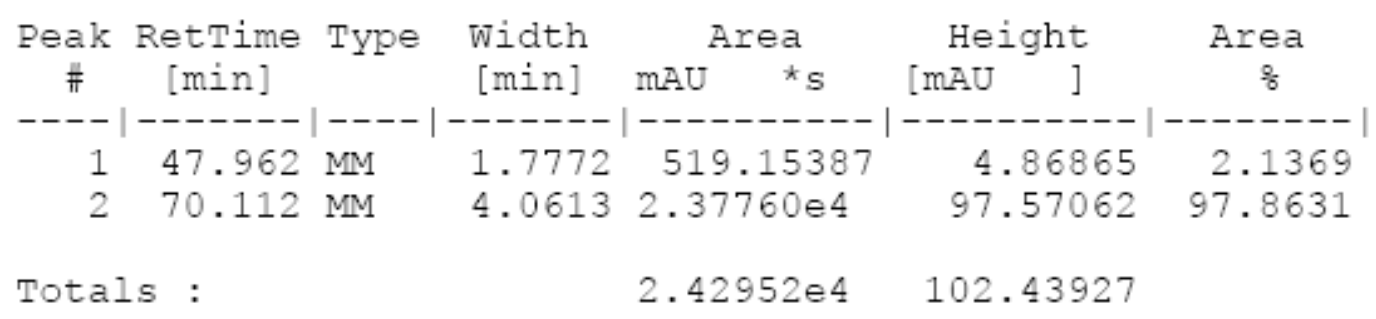



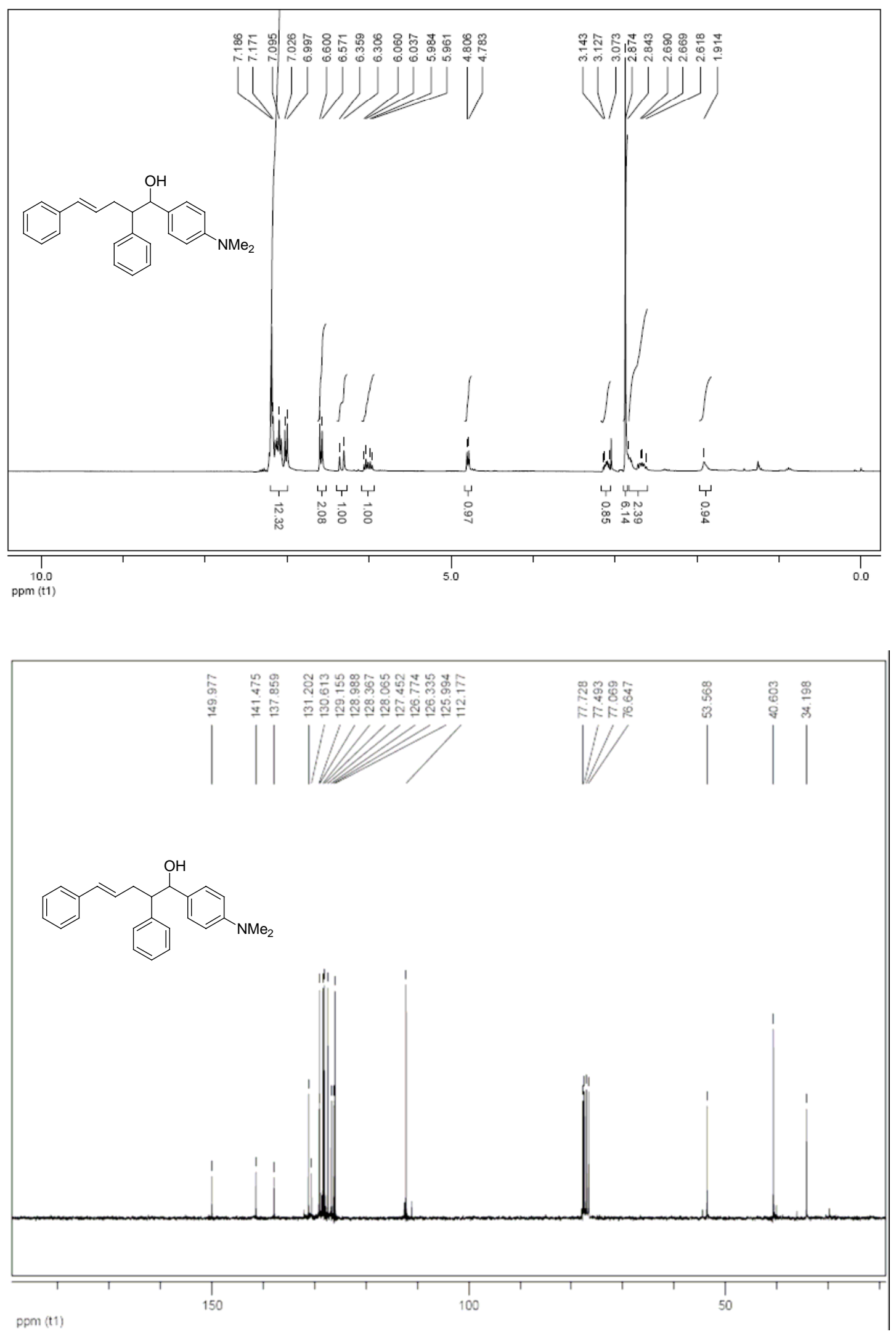

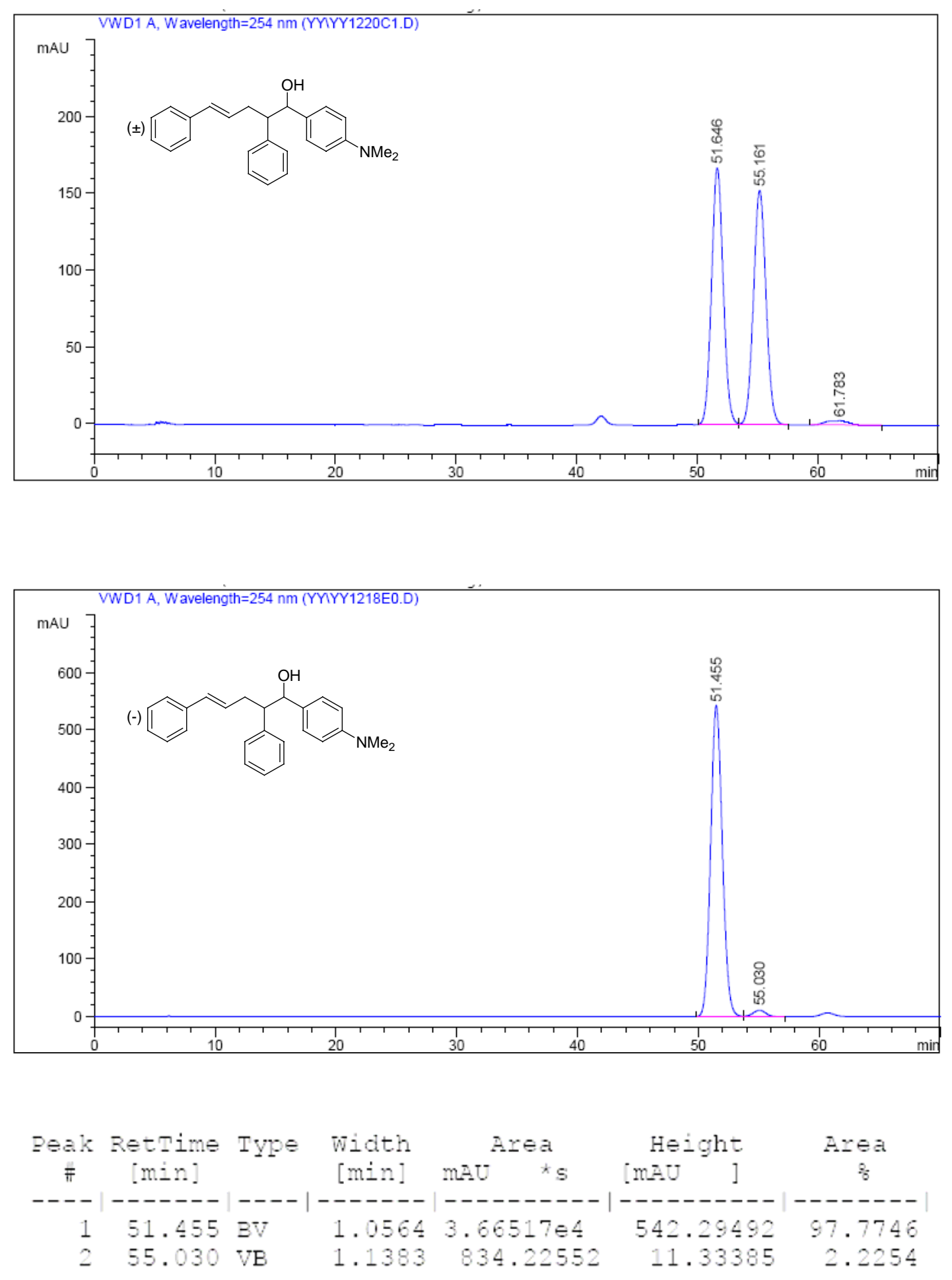

Totals:

$3.74860 e 4 \quad 553.62877$ 

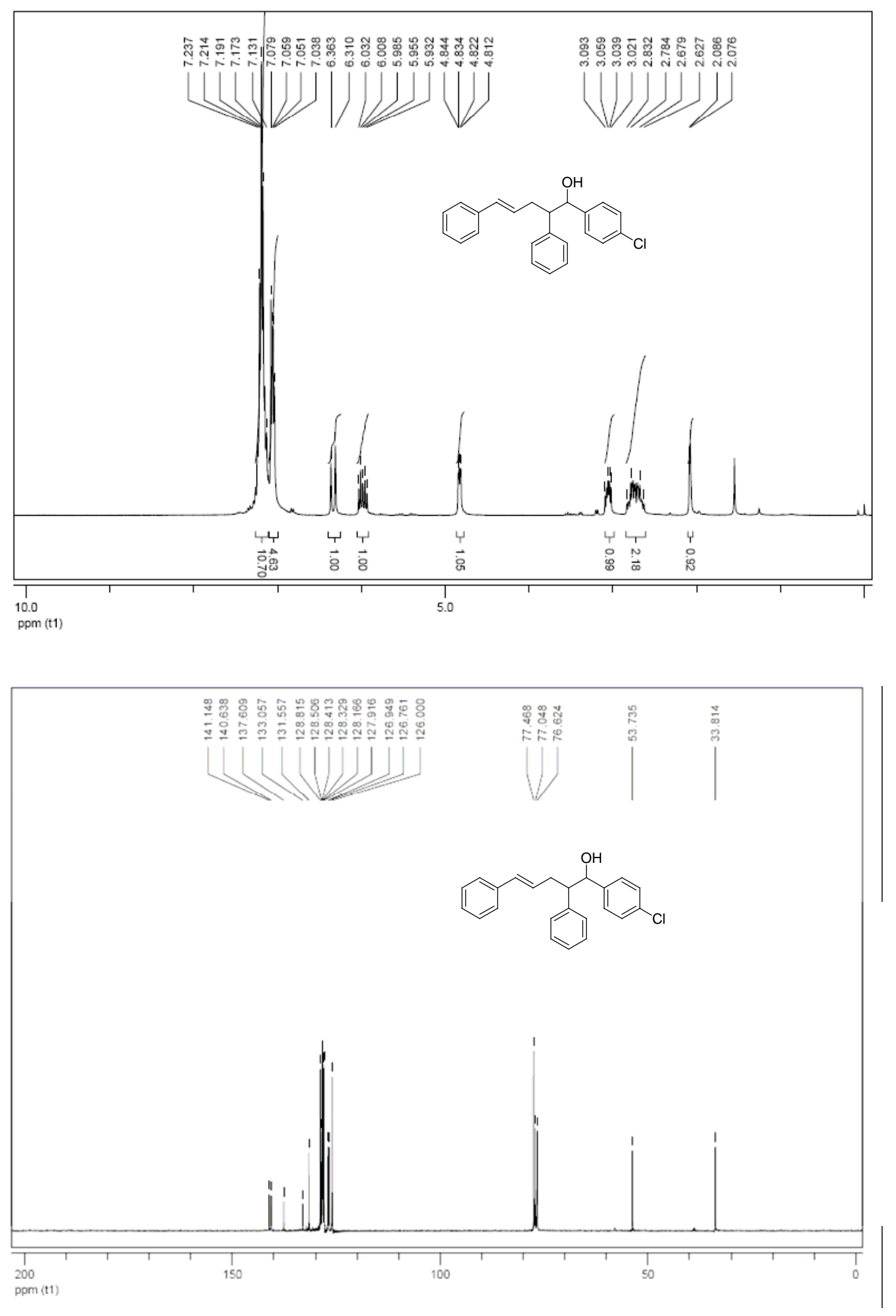

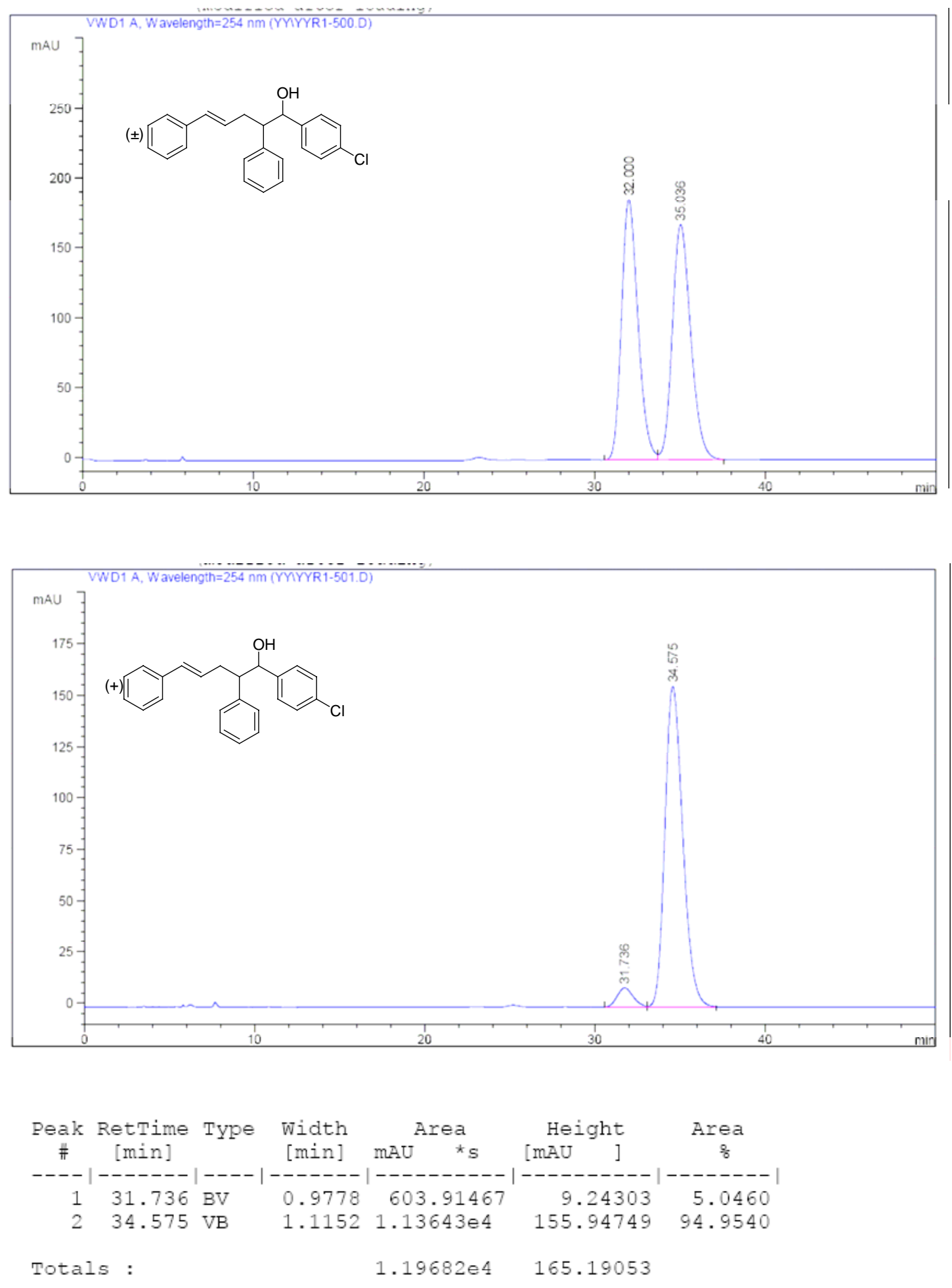

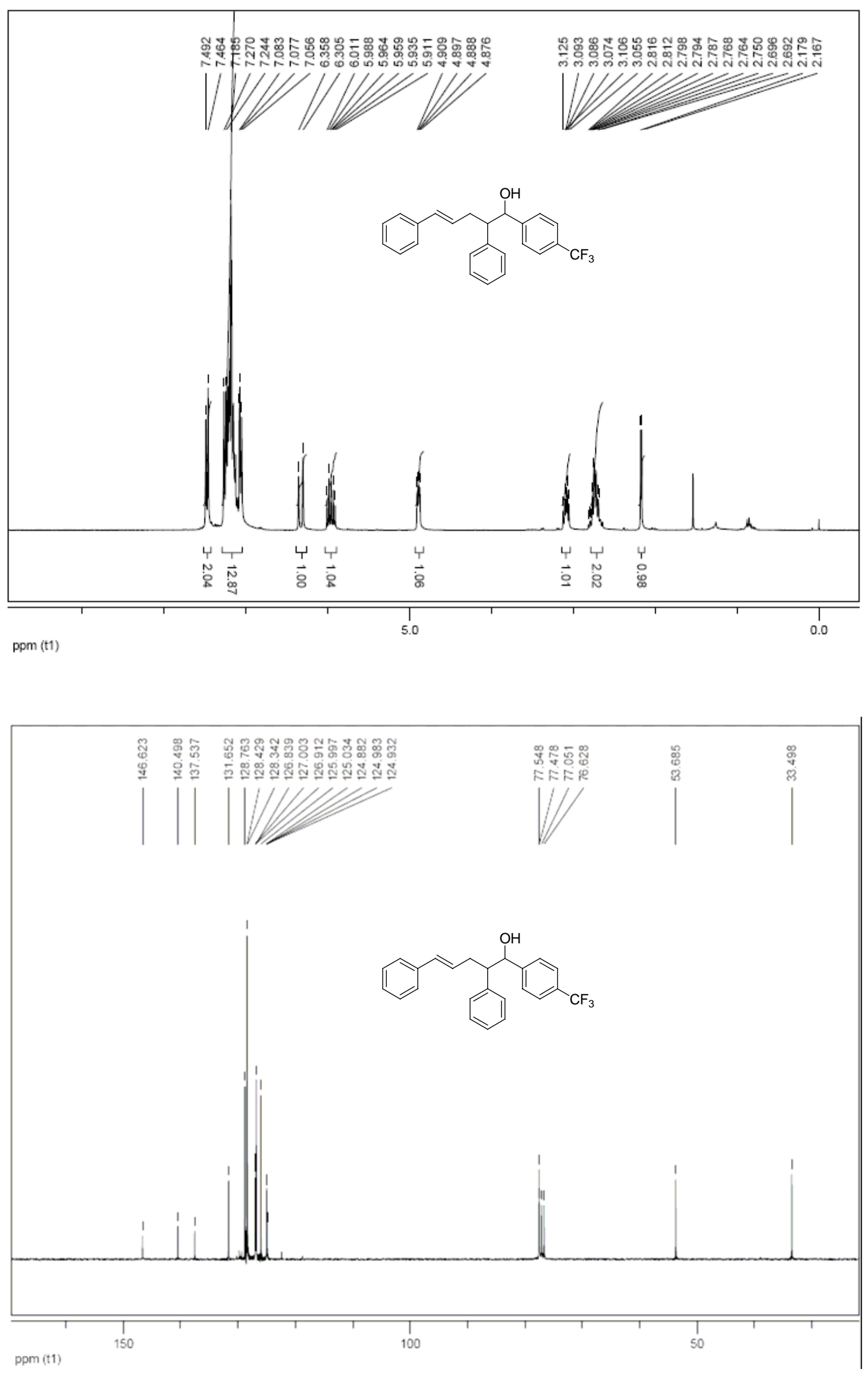

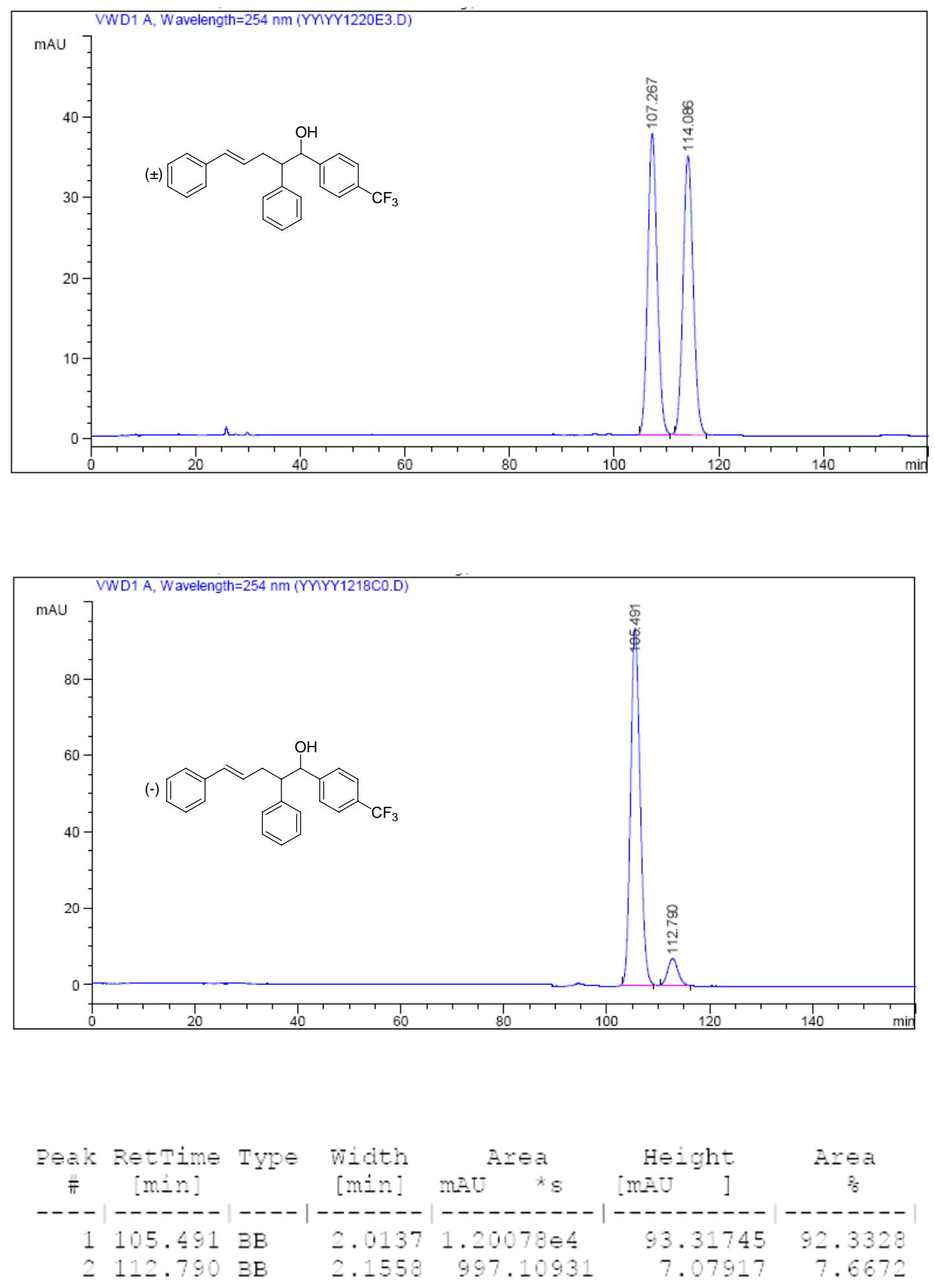

Totals :

$1.30049 e 4 \quad 100.39663$ 

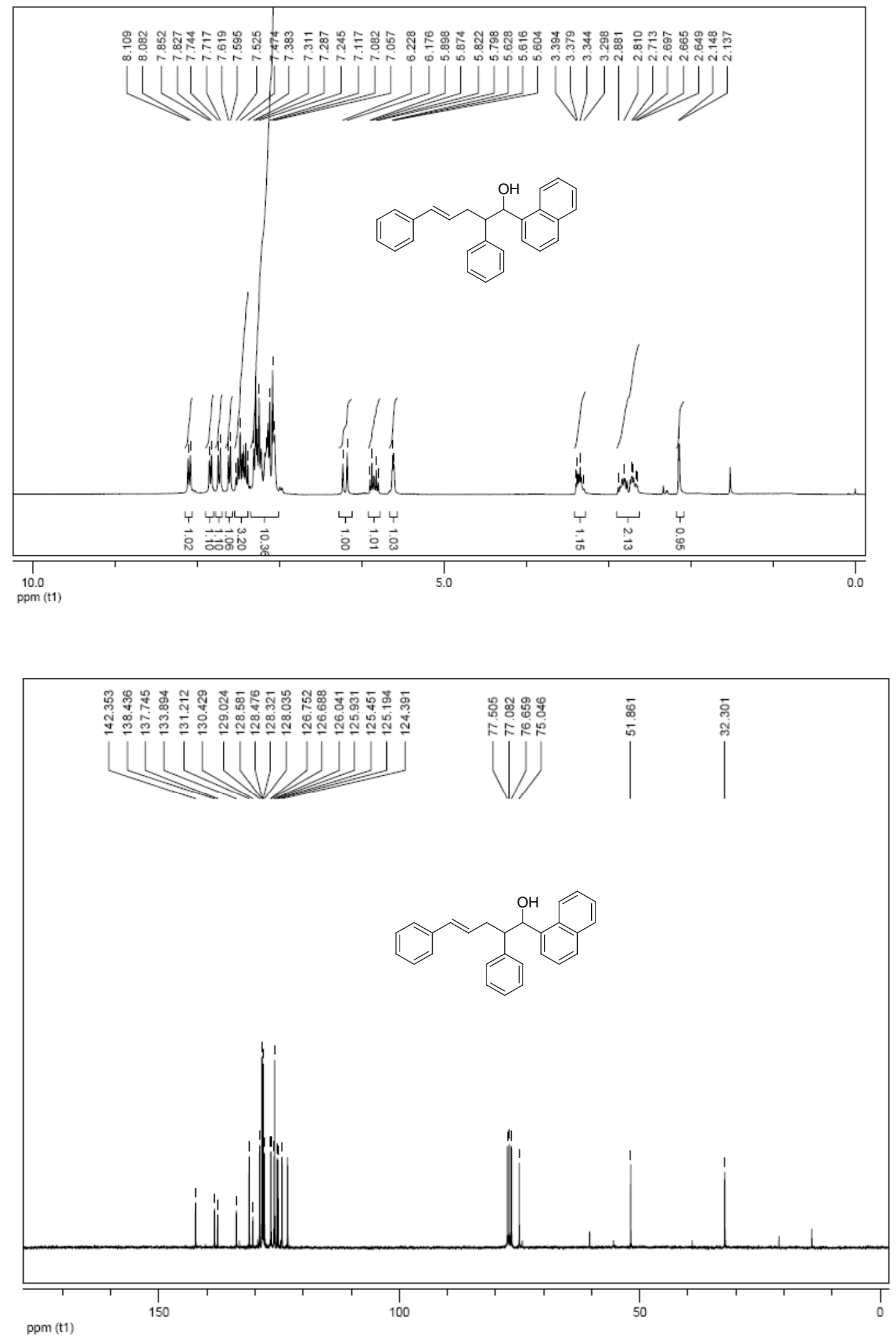

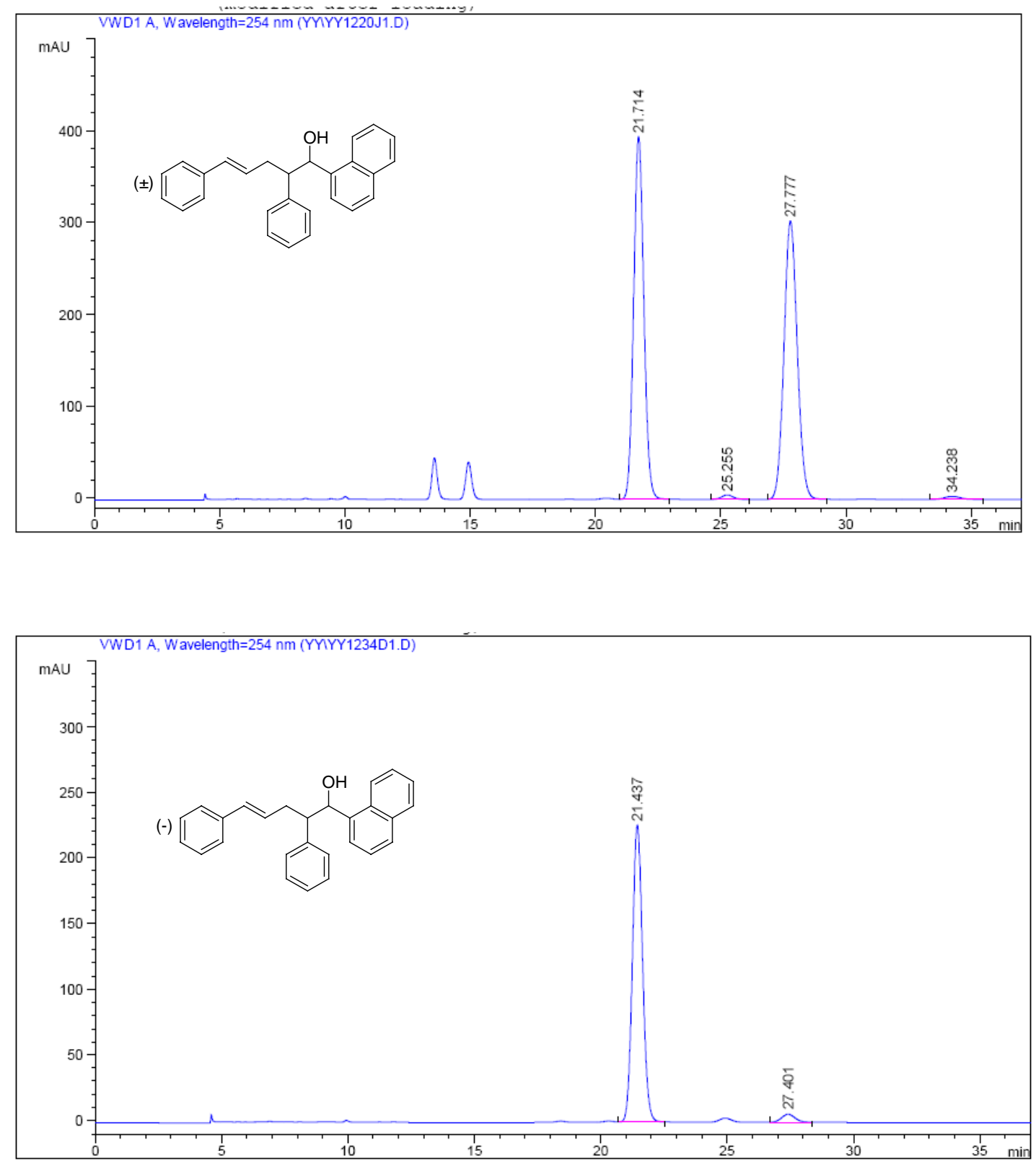

\begin{tabular}{|c|c|c|c|c|c|c|}
\hline$\underset{\#}{\text { Peak }}$ & RetTime & Type & Width & $\mathrm{mAU}^{\text {Area }}{ }^{\star} \mathrm{S}$ & $\begin{array}{r}\text { Height } \\
{[\mathrm{MAU}}\end{array}$ & $\begin{array}{c}\text { Area } \\
\frac{8}{6}\end{array}$ \\
\hline & & & & & & \\
\hline 1 & 21.437 & VB & 0.4335 & 6309.38379 & 225.97359 & $\begin{array}{r}96.5277 \\
3.4723\end{array}$ \\
\hline 2 & 27.401 & BB & 0.5748 & 226.96185 & 6.12714 & 3.4723 \\
\hline Tota & $s$ & & & 6536.34564 & 232.10072 & \\
\hline
\end{tabular}



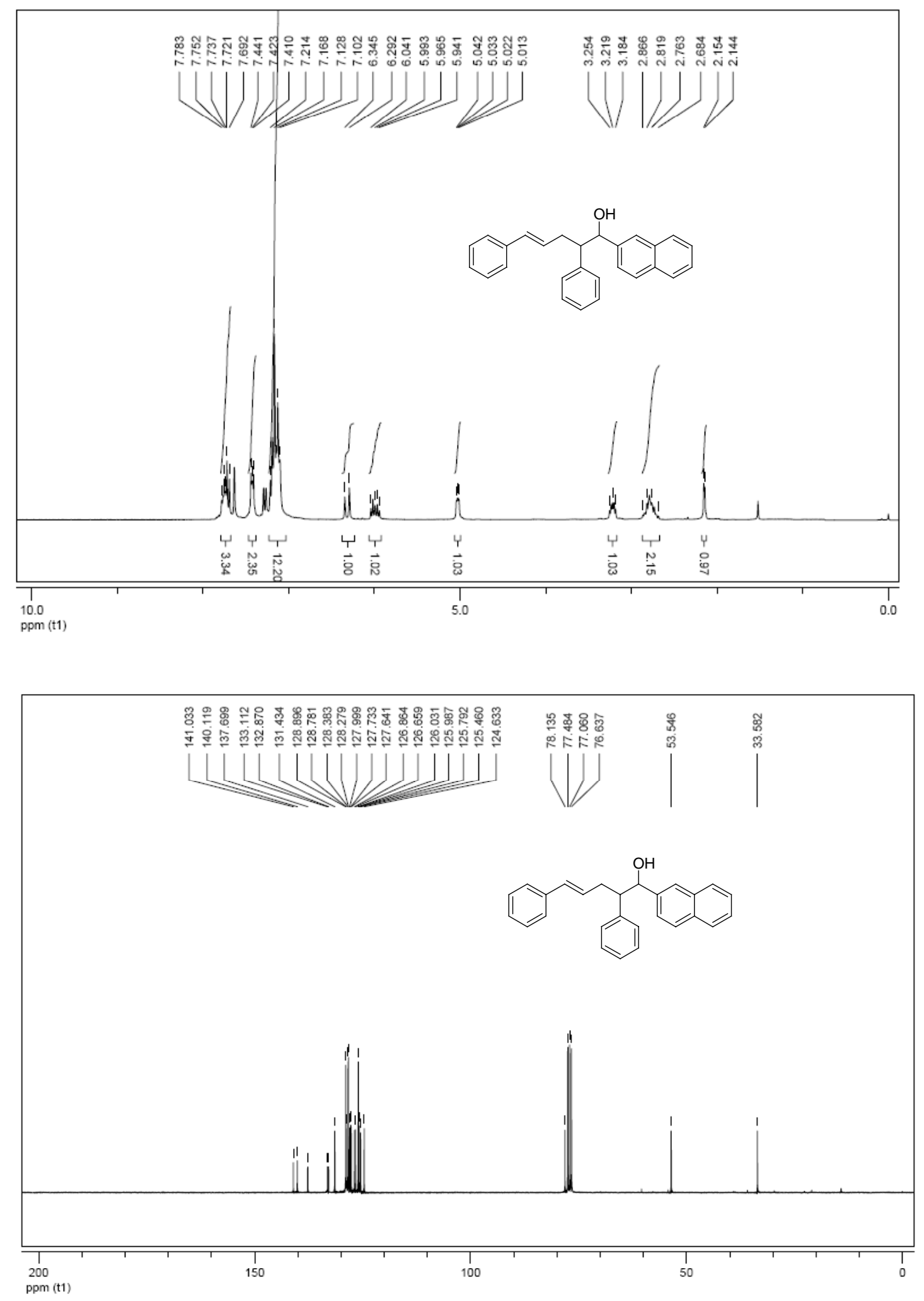

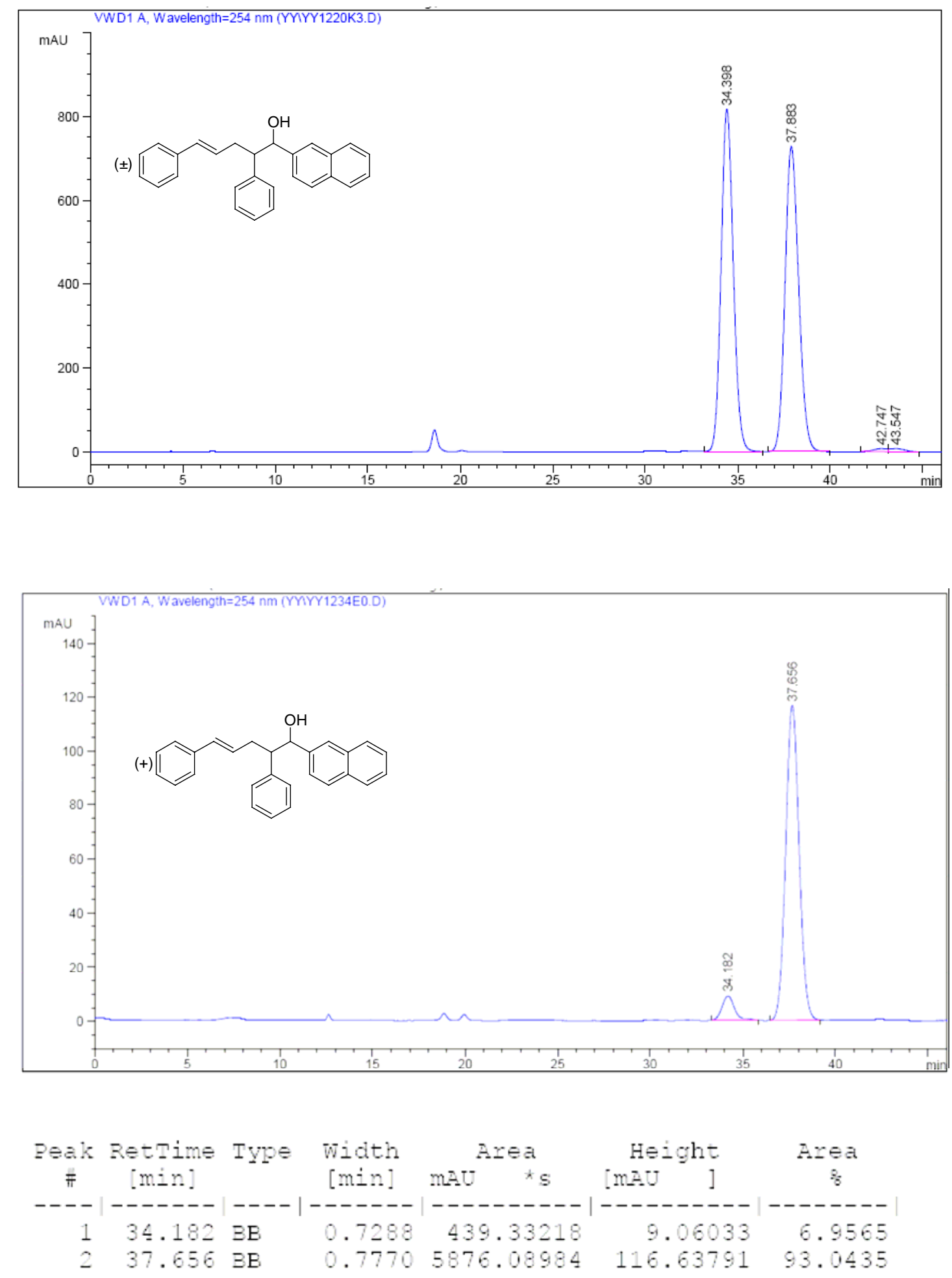

Totals :

$6315.42203 \quad 125.69824$ 

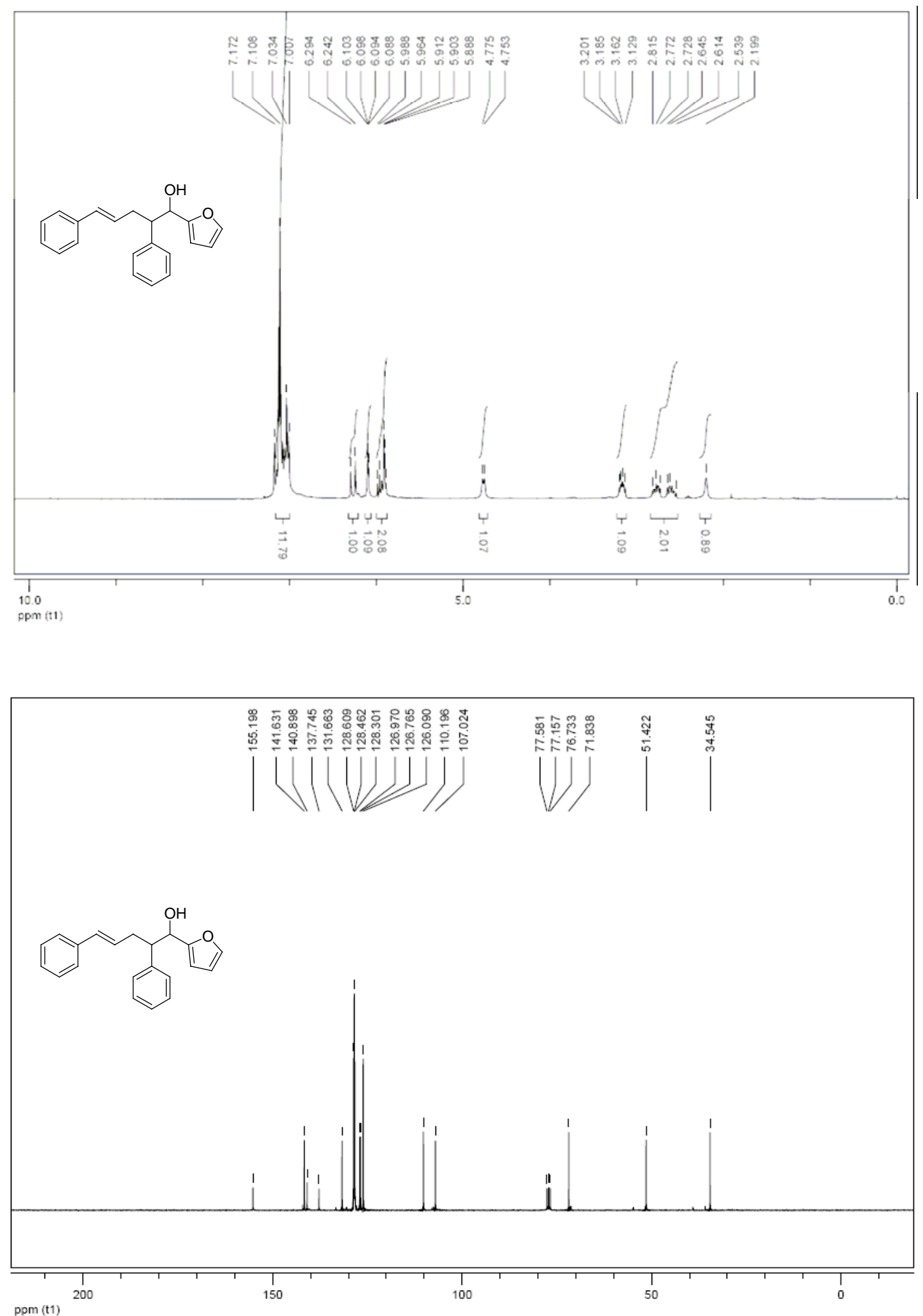

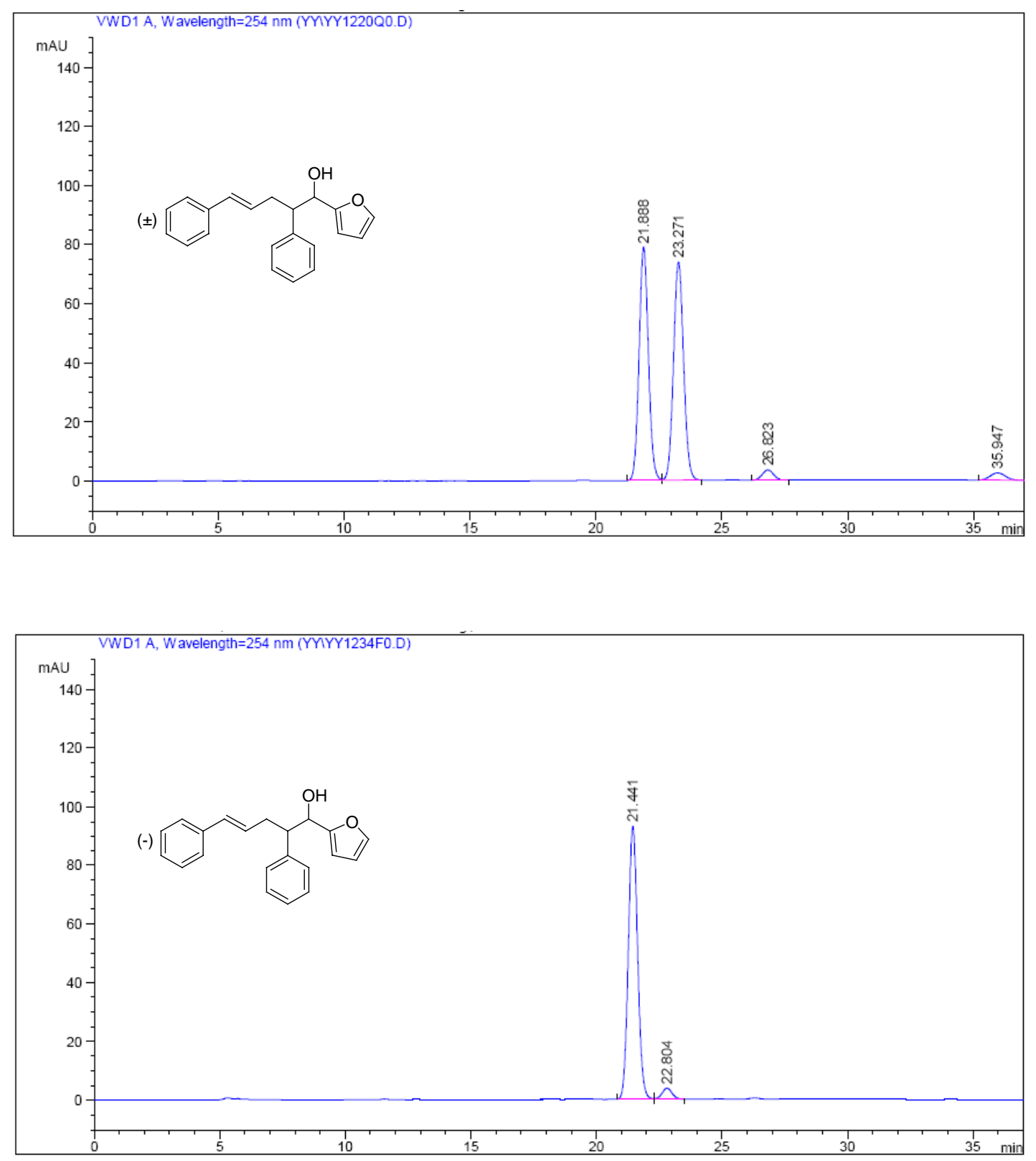

\begin{tabular}{|c|c|c|c|c|c|c|}
\hline geak & Retrime & Type & wic & Area & Height & Area \\
\hline 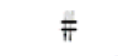 & [min] & & [min] & MAU & $\operatorname{mAU}$ & 8 \\
\hline$\frac{1}{2}$ & $\begin{array}{l}21.441 \\
22.804\end{array}$ & $\begin{array}{l}3 \mathrm{~B} \\
\mathrm{BB}\end{array}$ & $\begin{array}{l}0.3984 \\
0.4346\end{array}$ & $\begin{array}{r}2389.70532 \\
106.45329\end{array}$ & $\begin{array}{r}93.13506 \\
3.78242\end{array}$ & $\begin{array}{r}95.7353 \\
\leq .2647\end{array}$ \\
\hline-9 & & & & 2496.15862 & 747 & \\
\hline
\end{tabular}



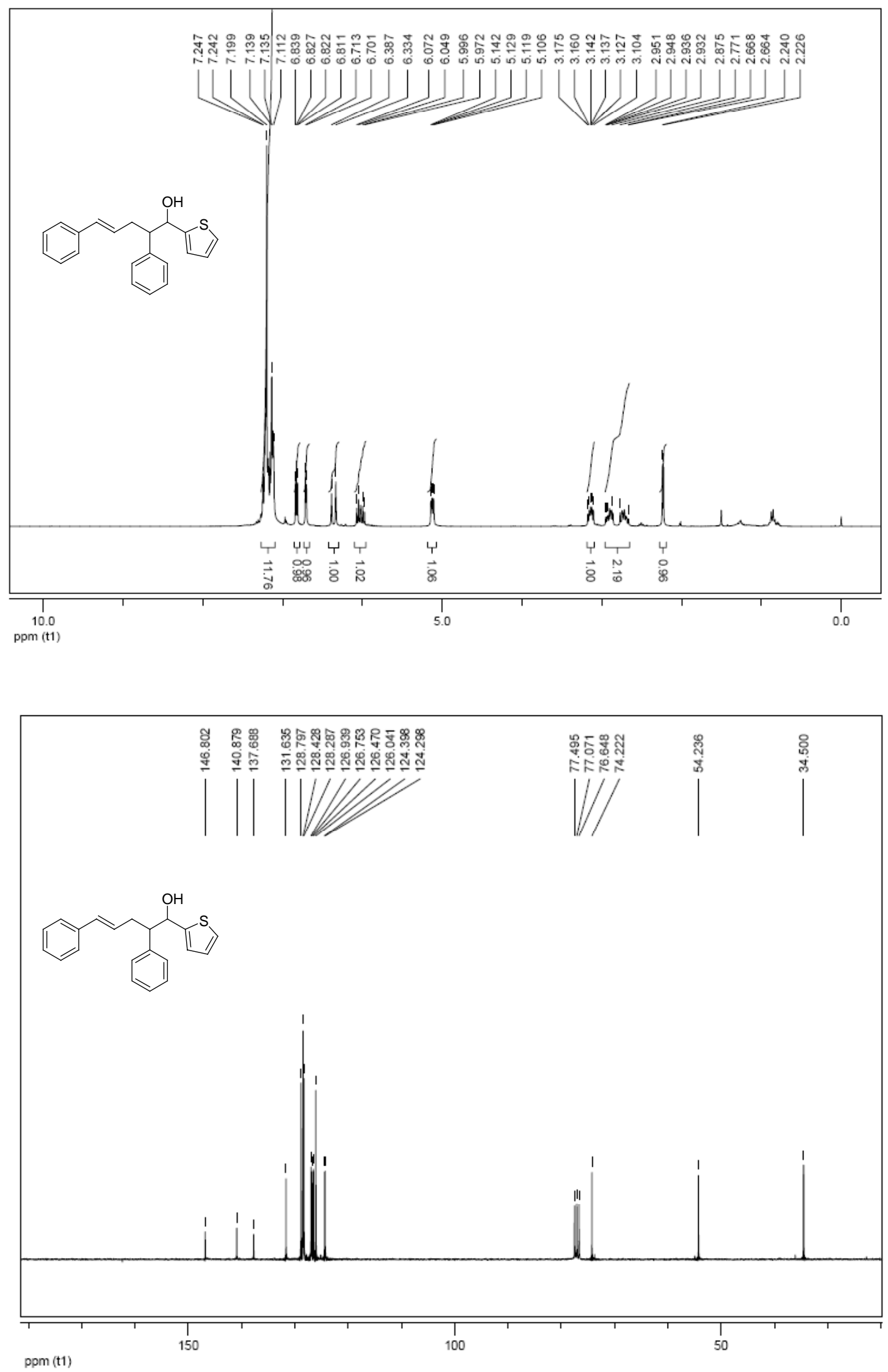

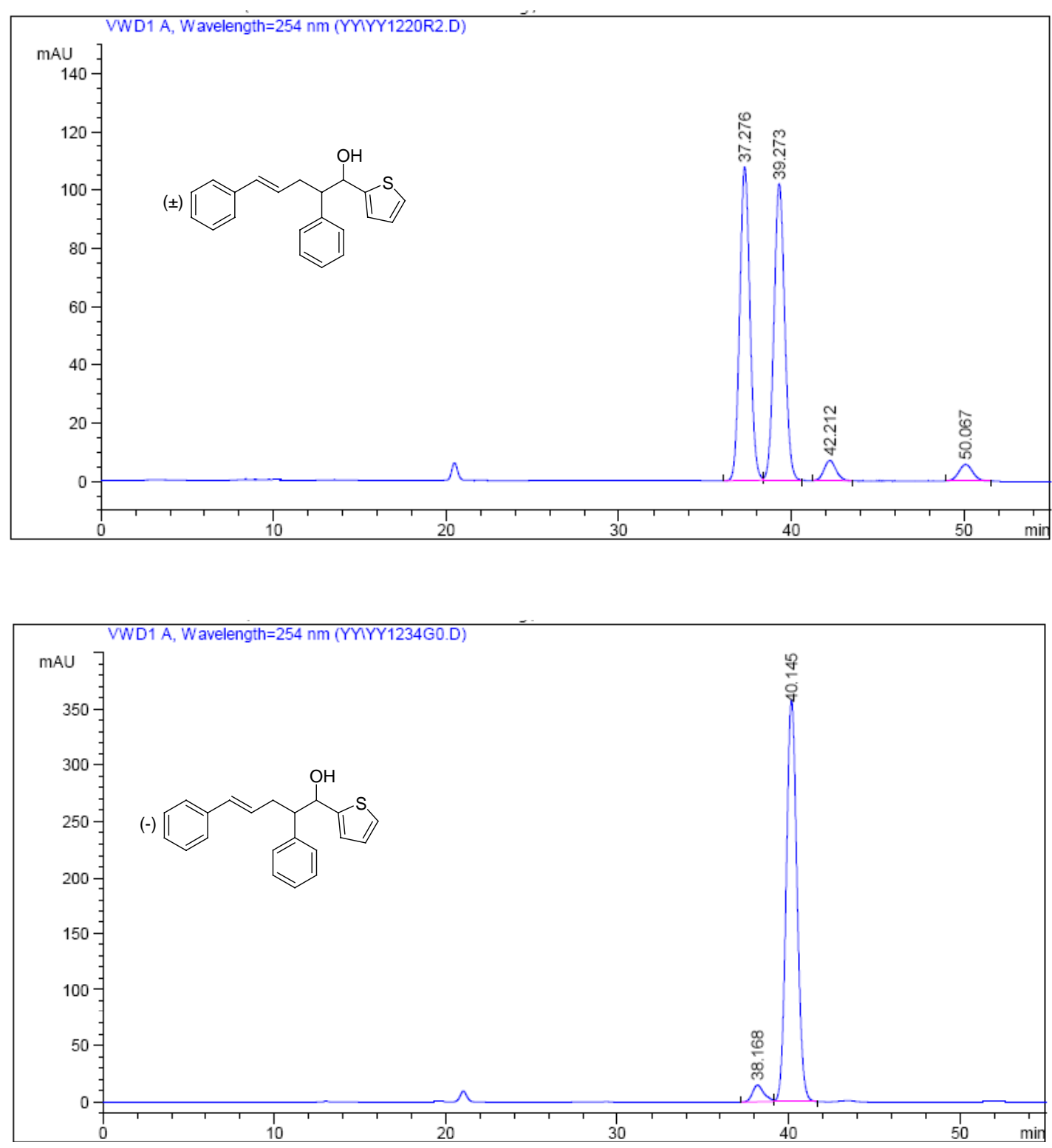

Signal 1: VWD1 A, Wavelength=254 nm

\begin{tabular}{|c|c|c|c|c|c|c|}
\hline \multirow{2}{*}{$\underset{\#}{\text { Peak }}$} & \multirow{2}{*}{$\begin{array}{l}\text { RetTime } \\
\text { [min] }\end{array}$} & \multirow{2}{*}{ Type } & \multirow{2}{*}{$\begin{array}{l}\text { Width } \\
\text { [min] }\end{array}$} & Area & Height & \multirow{2}{*}{$\begin{array}{c}\text { Area } \\
\frac{8}{6}\end{array}$} \\
\hline & & & & $\mathrm{mAU} \quad{ }^{*} \mathrm{~s}$ & {$[\mathrm{mAU}]$} & \\
\hline $\begin{array}{l}1 \\
2\end{array}$ & $\begin{array}{l}38.168 \\
40.145\end{array}$ & $\begin{array}{l}\text { BV } \\
\text { VB }\end{array}$ & $\begin{array}{l}0.6229 \\
0.5914\end{array}$ & $\begin{array}{c}707.35742 \\
1.55575 e 4\end{array}$ & $\begin{array}{r}14.80586 \\
358.00177\end{array}$ & $\begin{array}{r}4.3490 \\
95.6510\end{array}$ \\
\hline Tota] & & & & $1.62648 e 4$ & 372.80763 & \\
\hline
\end{tabular}



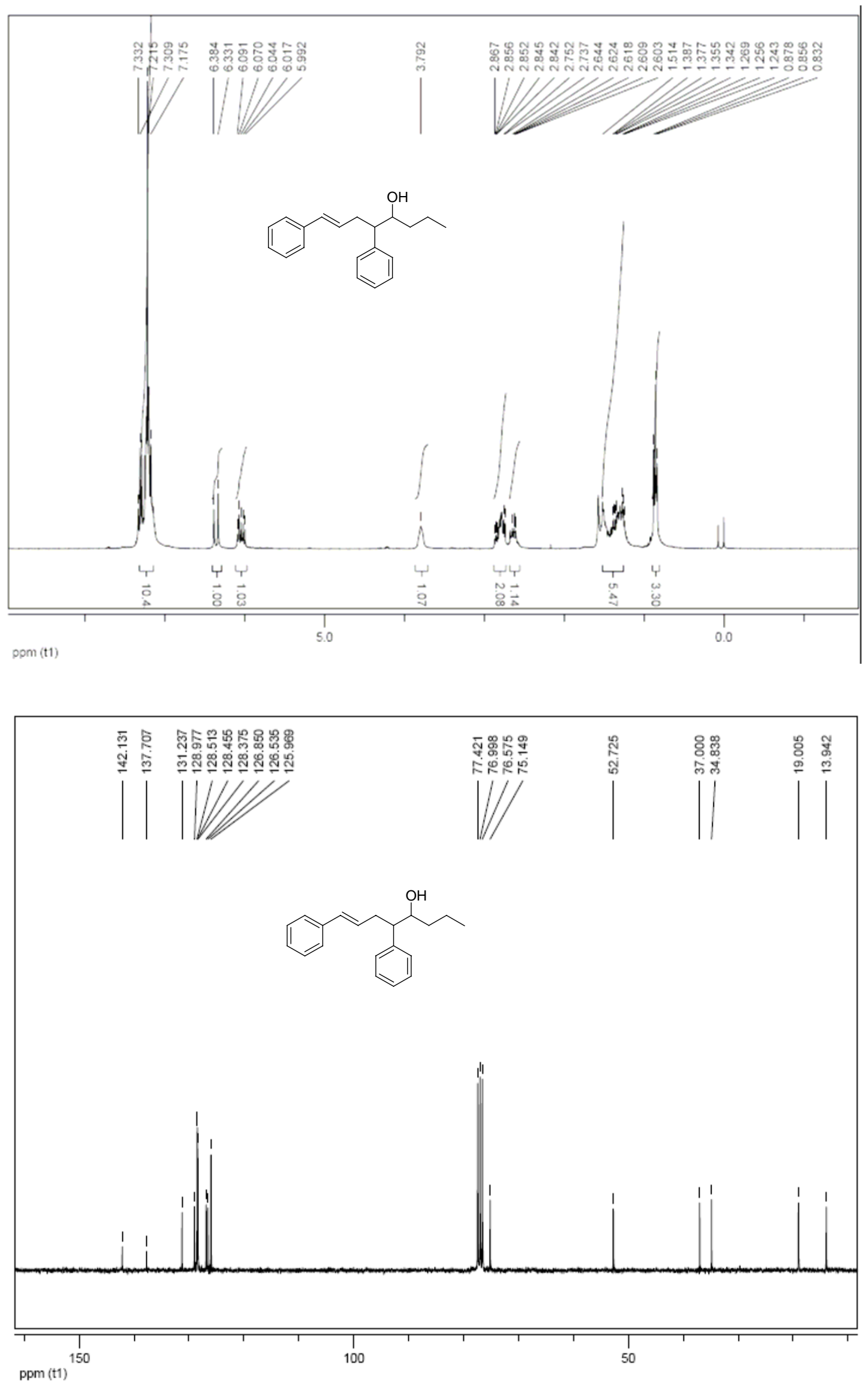

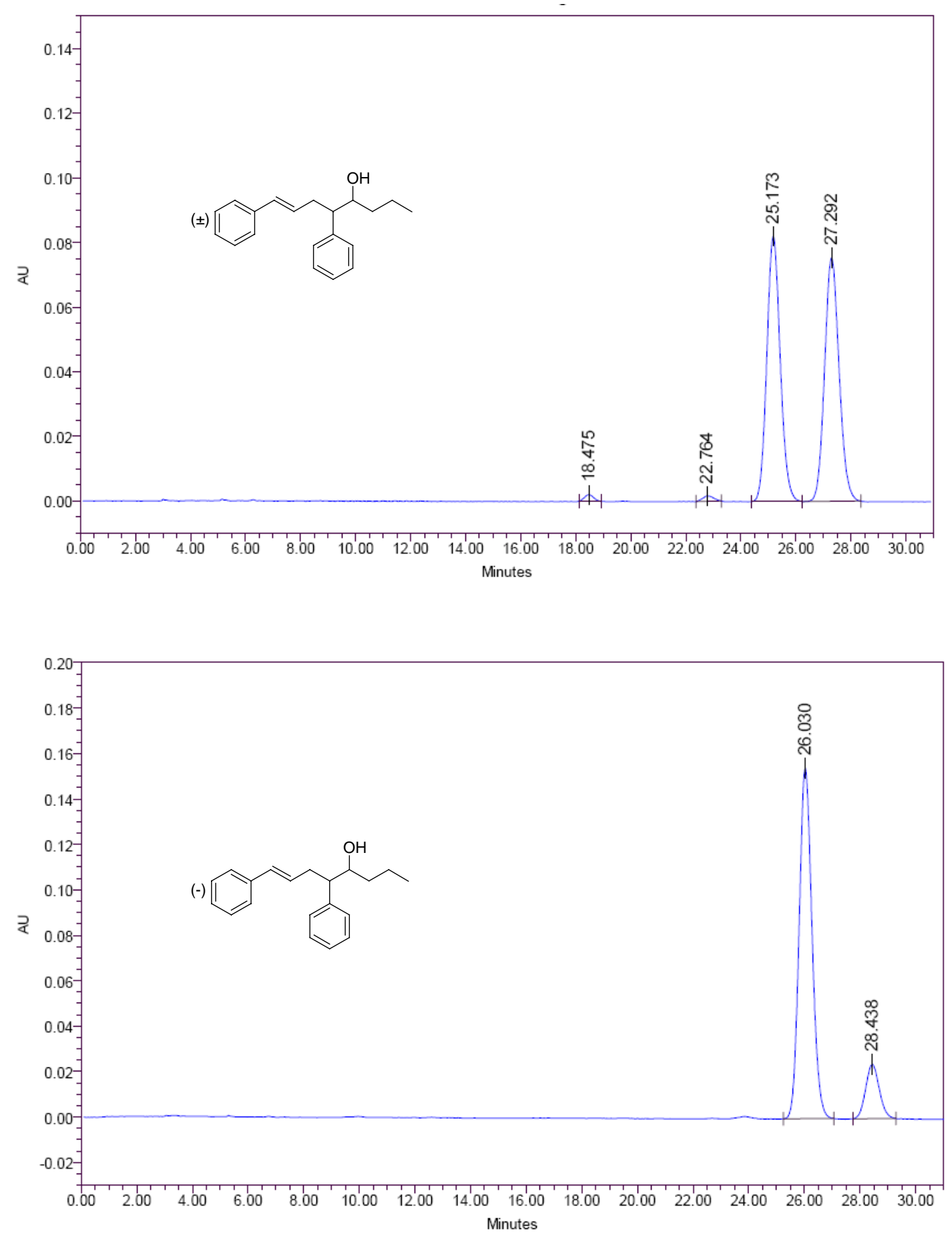

\begin{tabular}{|c|c|c|r|c|}
\hline & RT & Area & $\%$ Area & Height \\
\hline 1 & 26.030 & 4953540 & 85.88 & 154299 \\
\hline 2 & 28.438 & 814497 & 14.12 & 23914 \\
\hline
\end{tabular}

